Supporting Information

for

\title{
Density Functional Theory Investigations on Sulfur Ylide Promoted Cyclopropanation Reactions: Insights on
}

\section{Mechanism and Diastereoselection Issues}

\author{
Deepa Janardanan and Raghavan B. Sunoj * \\ Department of Chemistry \\ Indian Institute of Technology Bombay \\ Powai, Mumbai 400076, India
}

E-mail: sunoj@chem.iitb.ac.in

Fax: $91-22-2572-3480$ or $91-22-2576-7152$ 


\begin{tabular}{|c|c|c|}
\hline & Contents & Page No. \\
\hline & Details of PCM calculations employed in the study & S4 \\
\hline & $\begin{array}{l}\text { Reaction profile computed with solvent optimized } \\
\text { geometries }\end{array}$ & S4 \\
\hline & $\begin{array}{l}\text { Full list of citations for Gaussian } 98 \text { and Gaussian03 } \\
\text { (Reference } 17 \text { in the text) }\end{array}$ & S21-S22 \\
\hline & $\begin{array}{l}\text { Optimized Cartesian coordinates of transition states and } \\
\text { intermediates, product complexes, substituted ylides, enone } \\
(\mathrm{MeCH}=\mathrm{CH}-\mathrm{COMe} \text { ) for sulfur ylide mediated } \\
\text { cylopropanation reaction }\end{array}$ & S23-S75 \\
\hline & List of Figures & \\
\hline Figure S1 & $\begin{array}{l}\text { Reaction profiles for diastereomeric cyclopropane formation } \\
\text { from stabilized ylide } \mathbf{5}(\mathrm{R}=\mathrm{COMe}) \text { and enones with the } \\
\text { incorporation of solvent effects }\end{array}$ & S5 \\
\hline Figure S2 & $\begin{array}{l}\text { The B3LYP/6-31G* optimized geometries and relative } \\
\text { energies for cisoid and transoid intermediates from } \\
\text { semistabilized ylide } 2 \text { and stabilized ylide } 4\end{array}$ & S6 \\
\hline Figure S3 & $\begin{array}{l}\text { Reaction profiles for cyclopropane formation from stabilized } \\
\text { ylide } \mathbf{3}(\mathrm{R}=\mathrm{COOMe}) \text { and enone }\end{array}$ & S7 \\
\hline Figure S4 & $\begin{array}{l}\text { Reaction profiles for cyclopropane formation from stabilized } \\
\text { ylide } 4(\mathrm{R}=\mathrm{COPh}) \text { and enones }\end{array}$ & S8 \\
\hline Figure S5 & $\begin{array}{l}\text { The B3LYP/6-31G }{ }^{*} \text { optimized geometries and relative } \\
\text { energies for transoid TSs from stabilized ylide } 4\end{array}$ & S9 \\
\hline \multirow[t]{2}{*}{ Figure S6 } & $\begin{array}{l}\text { NPA charges computed at the B3LYP/6-311+G }{ }^{* *} / / \mathrm{B} 3 \mathrm{LYP} / 6- \\
31 \mathrm{G}^{*} \text { on developing charge centers for different addition } \\
\text { transition states for ylide } 5\end{array}$ & $\mathrm{~S} 10$ \\
\hline & List of Schemes & \\
\hline Scheme S1 & $\begin{array}{l}\text { Torsional rotation possibilities for syn and anti betaines of } \\
\text { stabilized ylides }\end{array}$ & S11 \\
\hline
\end{tabular}




\begin{tabular}{|c|c|c|}
\hline & List of Tables & \\
\hline Table S1 & $\begin{array}{l}\text { Relative energies of intermediates and products (E in kcal } \\
\text { mol }^{-1} \text { ) computed at the PCM/B3LYP/6-311+G }{ }^{* *} / / \mathrm{B} 3 \mathrm{LYP} / 6- \\
31 \mathrm{G}^{*} \text { level of theory for the formation of cyclopropanes } \\
\text { using acetonitrile solvent }\end{array}$ & S12 \\
\hline Table S2 & $\begin{array}{l}\text { Acctivation barriers for addition TSs (high energy } \\
\text { conformers) (in kcal } \mathrm{mol}^{-1} \text { ) computed at the PCM/B3LYP/6- } \\
311+\mathrm{G}^{* *} / / \mathrm{B} 3 \mathrm{LYP} / 6-31 \mathrm{G}^{*} \text { level of theory for the formation of } \\
\text { cyclopropanes using acetonitrile solvent }\end{array}$ & S12 \\
\hline Table S3 & $\begin{array}{l}\text { Comparison of energies for various stationary points along } \\
\text { the anti pathway of ylide } \mathbf{3}\end{array}$ & S13 \\
\hline Table S4 & $\begin{array}{l}\text { IRC profiles for addition and elimination TSs for sulfur ylide } \\
\text { mediated cyclporopnation reaction }\end{array}$ & S14-S21 \\
\hline
\end{tabular}




\section{Details of PCM calculations}

1. Single-point energy calculations: The default options available with the PCM as implemented in Gaussian98 were used (UAHF radii) using acetonitrile as the dielectric continuum at the B3LYP/6-311+G $\mathrm{G}^{* *}$ level for calculating single-point energies for ALL stationary points. 2. Geometry Optimizations: In selected cases, where geometry optimizations were performed in acetonitrile dielectric continuum, the default options implemented in PCM version of Gaussian03 were used (UA0 radii). Again, single-point energies were computed at the B3LYP/6-311+ $\mathrm{G}^{* *}$ level only using Gaussian98 so as to enable a direct comparison of energies from both sets of calculations.

\section{Reaction profile computed with solvent optimized geometries}

To verify how good the gas phase reaction profile is as compared to full geometry optimization with inclusion of continuum solvation effects, we have re-optimized the gas phase minima and TSs for a representative case of stabilized ylide 5 (syn and anti pathways) in continuum acetonitrile medium using the PCM model at the B3LYP/6-31G* level as implemented in Gaussian03. It is found that the energy profile obtained at the $\mathrm{PCM}\left(\mathrm{CH}_{3} \mathrm{CN}\right) / \mathrm{B} 3 \mathrm{LYP} / 6-311+\mathrm{G}^{* *} / / \mathrm{PCM}\left(\mathrm{CH}_{3} \mathrm{CN}\right) / \mathrm{B} 3 \mathrm{LYP} / 6-31 \mathrm{G}^{*}$ level is in excellent agreement with that obtained from the gas phase optimized geometries (the energy profile is shown below as Figure $\mathrm{S} 1$ ). The $\Delta \mathrm{E}$ responsible for stereoselection is found to be exactly the same in both the gas phase and in the condensed phase for this representative

ylide $\left(4.7 \mathrm{kcal} \mathrm{mol}^{-1}\right.$ for ylide 5). The calculations additionally confirm that syn and anti pathways are nearly isoenergetic as far as the initial addition step is concerned, an observation quite similar to that obtained from the gas phase computations. Hence, it can be concluded that the energy information obtained at the $\mathrm{PCM}\left(\mathrm{CH}_{3} \mathrm{CN}\right) / \mathrm{B} 3 \mathrm{LYP} / 6$ $311+\mathrm{G}^{* *} / / \mathrm{B} 3 \mathrm{LYP} / 6-31 \mathrm{G}^{*}$ level is sufficiently reliable for systems considered in the present study. 


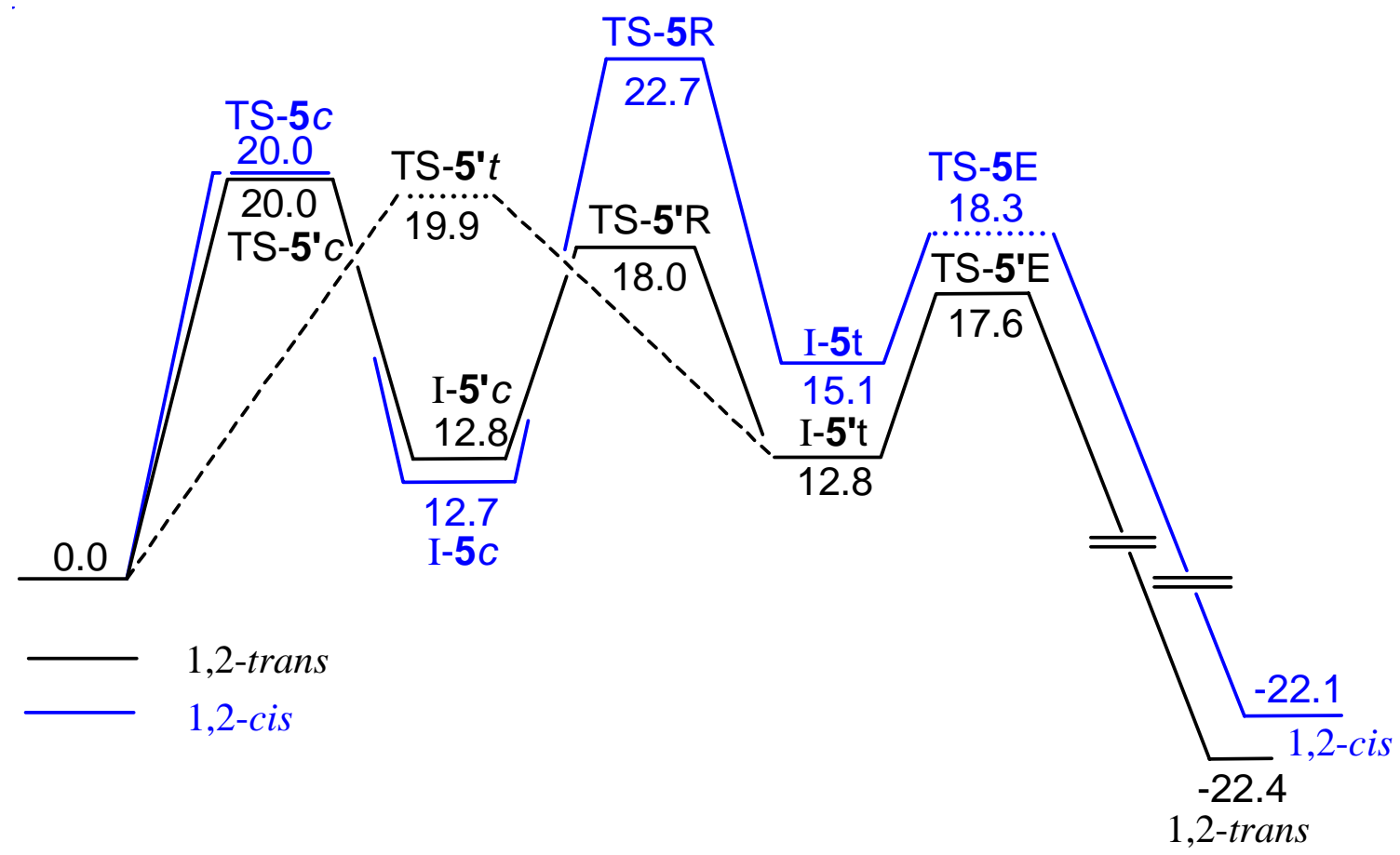

Figure S1. Reaction profiles for diastereomeric cyclopropane formation from stabilized ylide $5(\mathrm{R}=\mathrm{COMe})$ and enones incorporating solvent effects. Activation energies in $\mathrm{CH}_{3} \mathrm{CN}$ (kcal $\mathrm{mol}^{-1}$ ) at the PCM/B3LYP/6-311+G ${ }^{* *} / / \mathrm{PCM} / \mathrm{B} 3 \mathrm{LYP} / 6-31 \mathrm{G}^{*}$ level. Energies are reported with respect to isolated reactants. Only for TS-5E (…) relative energy is at the $\mathrm{PCM}\left(\mathrm{CH}_{3} \mathrm{CN}\right) / \mathrm{B} 3 \mathrm{LYP} / 6-311+\mathrm{G}^{* *} / / \mathrm{PCM}\left(\mathrm{CH}_{3} \mathrm{CN}\right) / \mathrm{mPW} 1 \mathrm{PW} 91 / 6-31 \mathrm{G}^{*}$ level. 


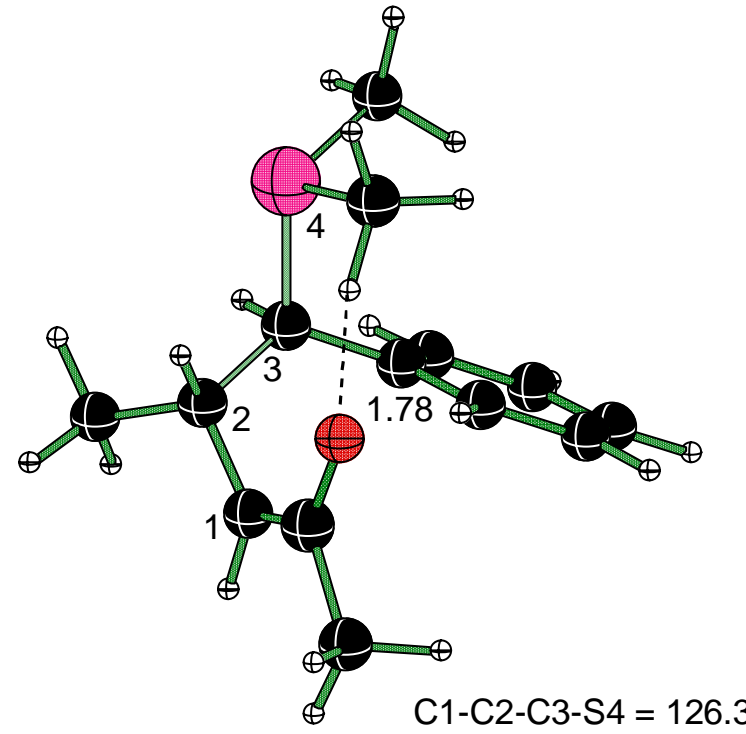

$\mathrm{I}-2 t(-2.2)$

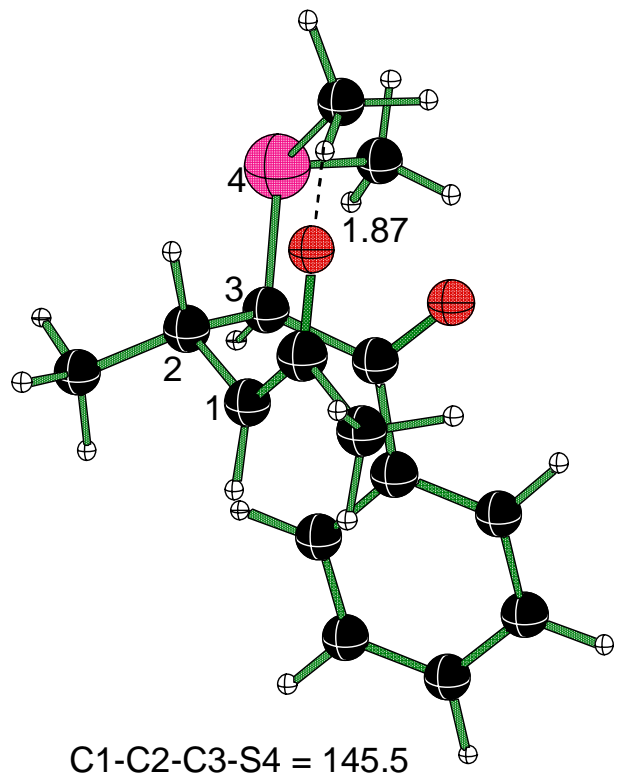

$\mathrm{I}-4 t(22.7)$

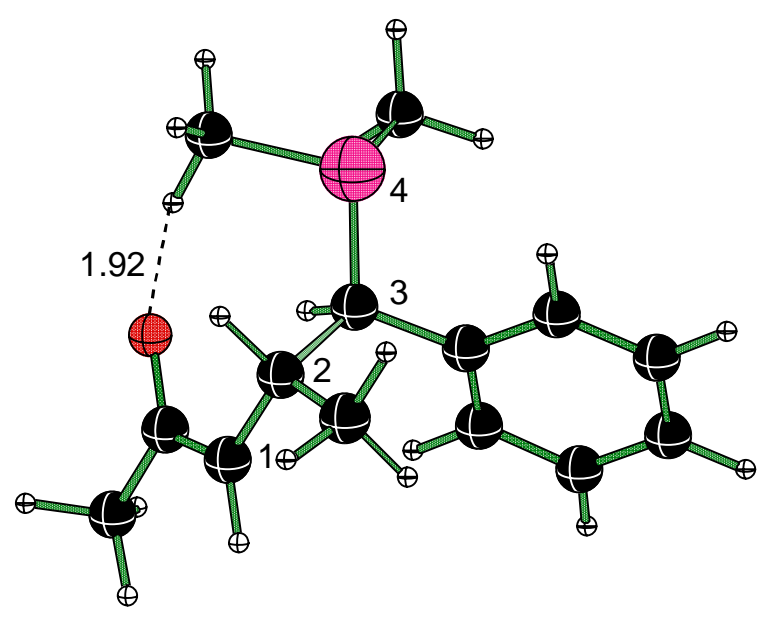

C1-C2-C3-S4 = 142.3
$\mathrm{I}-2 ' t(-3.9)$

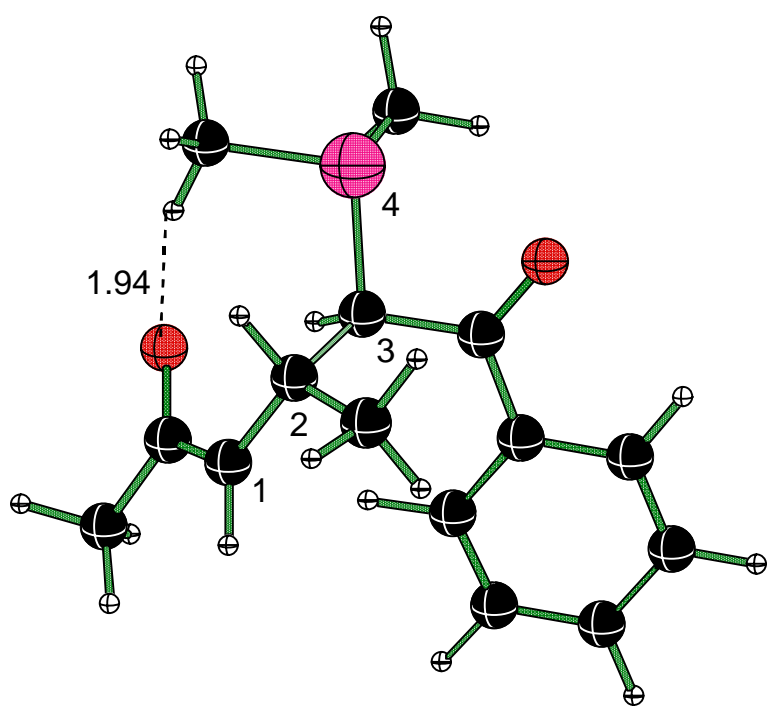

C1-C2-C3-S4 = 135.1

$\mathrm{I}-4 ' t(18.3)$

Figure S2. The B3LYP/6-31G ${ }^{*}$ optimized geometries and relative energies (in parentheses) for cisoid and transoid intermediates from semistabilized ylide $\mathbf{2}$ and stabilized ylide 4 [Distances in $\AA$, Angles in ${ }^{\circ}$, Energies in $\mathrm{kcal} \mathrm{mol}^{-1}$. Atom colors: Black $-\mathrm{C}$, Pink -S, Red -O. Energies refer to $\triangle \mathrm{E}$ in $\mathrm{CH}_{3} \mathrm{CN}$ computed at the PCM/B3LYP/6$311+\mathrm{G}^{* * *} / / \mathrm{B} 3 \mathrm{LYP} / 6-31 \mathrm{G}^{*}$ level, Energies are reported with respect to isolated reactants]. 


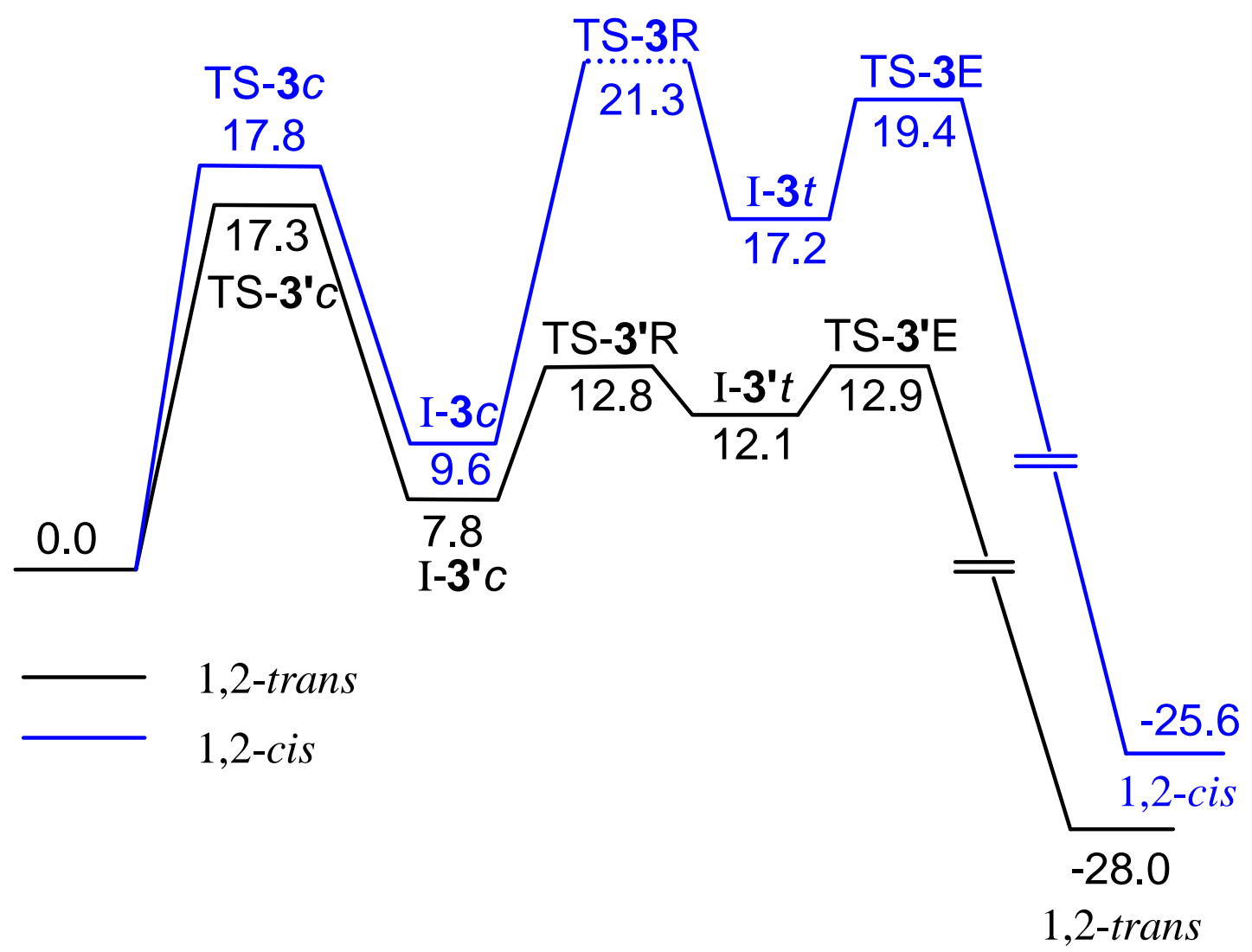

Figure S3. Reaction profiles for cyclopropane formation from stabilized ylide $\mathbf{3}$ $(\mathrm{R}=\mathrm{COOMe})$ and enone. Activation energies in $\mathrm{CH}_{3} \mathrm{CN}\left(\mathrm{kcal} \mathrm{mol}^{-1}\right)$ are obtained with the PCM/B3LYP/6-311+G**//B3LYP/6-31G ${ }^{*}$ level. Energies are reported with respect to isolated reactants [Only for TS-3R (…) the relative energy is at the PCM/B3LYP/6$311+\mathrm{G}^{* *} / / \mathrm{mPW} 1 \mathrm{PW} 91 / 6-31 \mathrm{G}^{*}$ level]. 


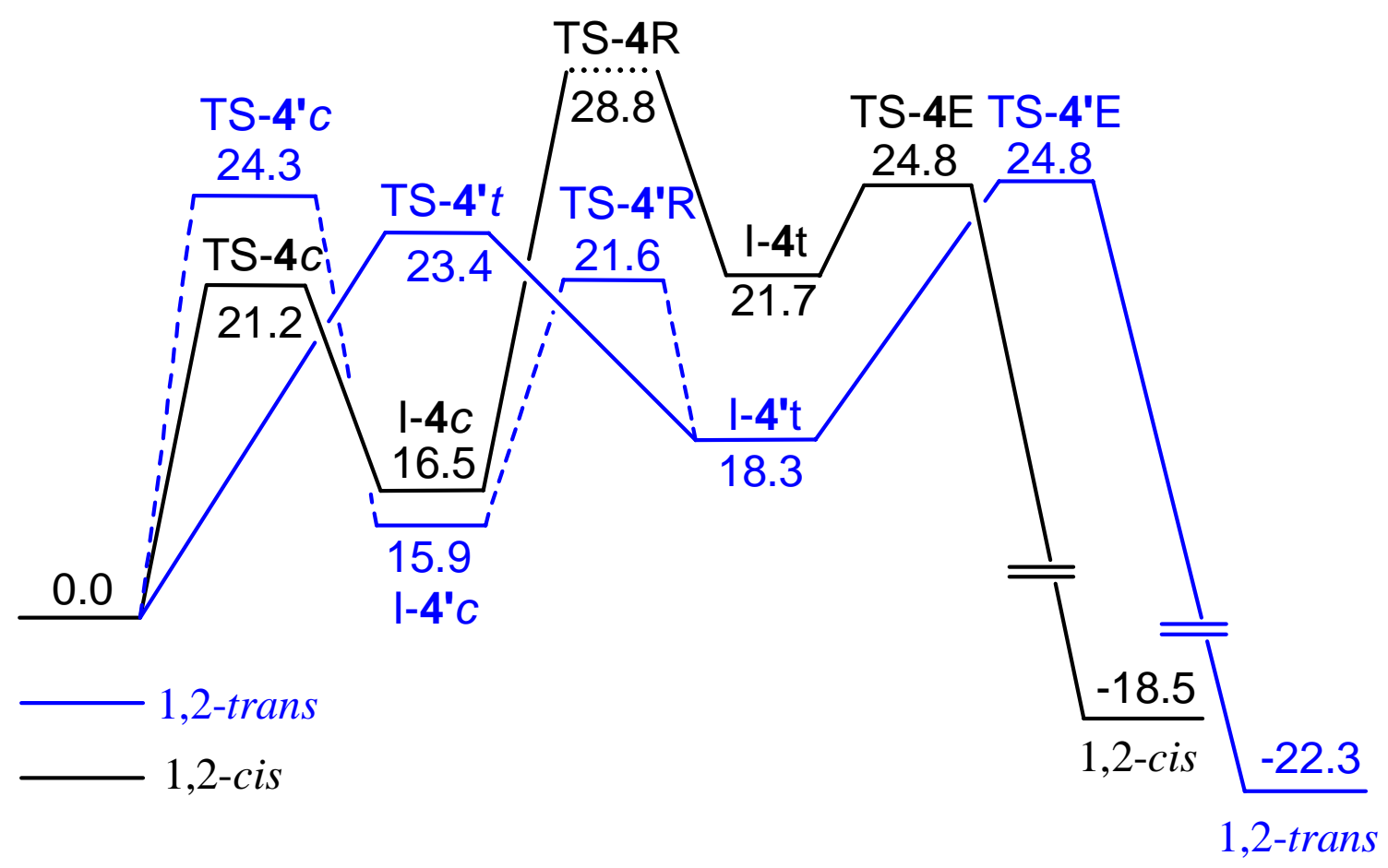

Figure S4. Reaction profiles for cyclopropane formation from stabilized ylide 4 ( R = $\mathrm{COPh})$ and enones. Activation energies in $\mathrm{CH}_{3} \mathrm{CN}\left(\mathrm{kcal} \mathrm{mol}^{-1}\right)$ are obtained with the $\mathrm{PCM} / \mathrm{B} 3 \mathrm{LYP} / 6-311+\mathrm{G}^{* *} / / \mathrm{B} 3 \mathrm{LYP} / 6-31 \mathrm{G}^{*}$ level. Energies are reported with respect to isolated reactants [Only for TS-4R (…) the relative energy is at the PCM/B3LYP/6$311+\mathrm{G}^{* *} / / \mathrm{mPW} 1 \mathrm{PW} 91 / 6-31 \mathrm{G}^{*}$ level]. 


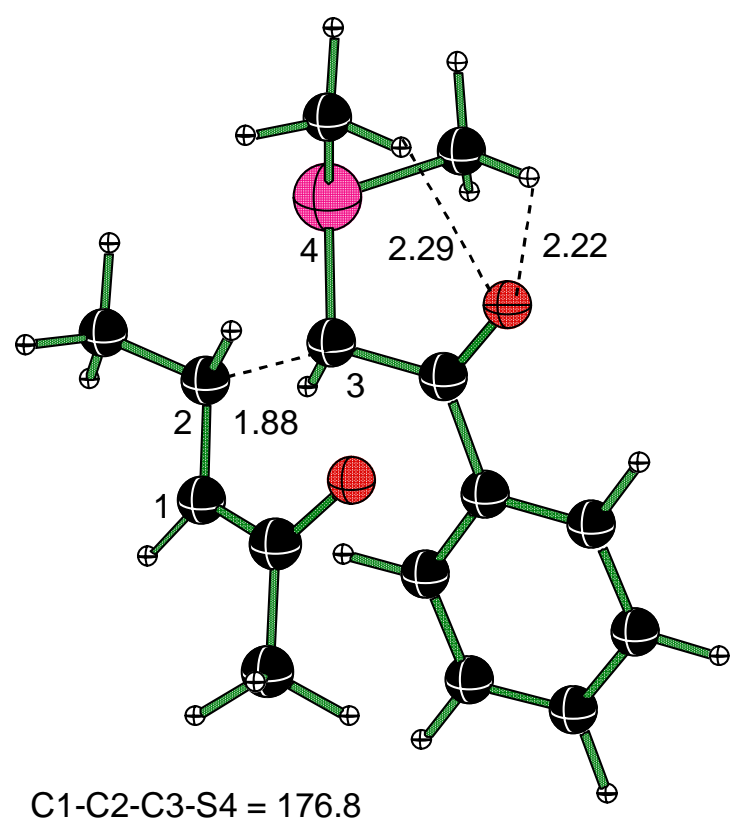

TS-4t (31.1)

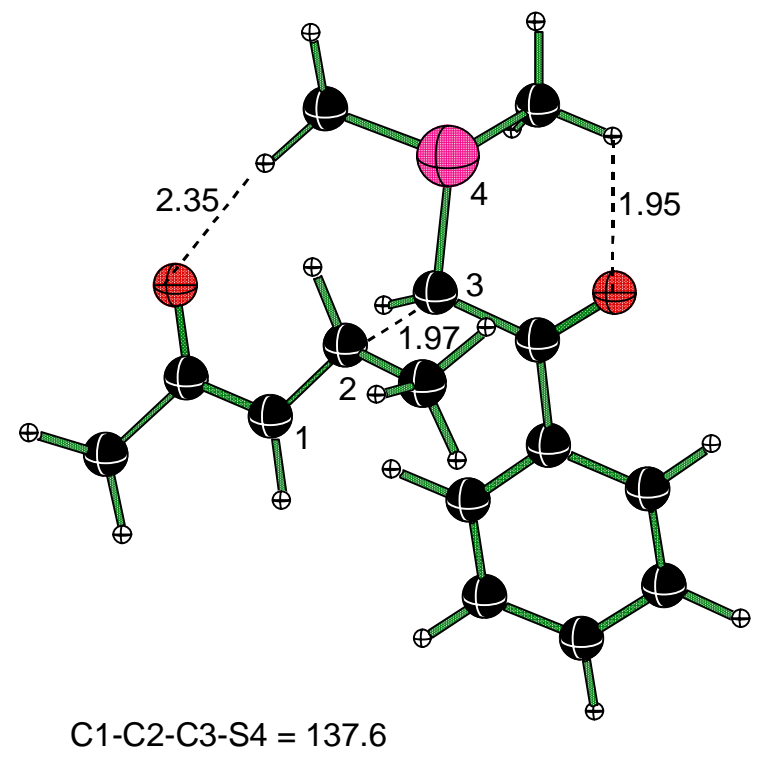

TS-4't (23.4)

Figure S5. The B3LYP/6-31G ${ }^{*}$ optimized geometries and relative energies (in parentheses) for transoid TSs from stabilized ylide 4 [Distances in $\AA$, Angles in ${ }^{\circ}$, Energies in kcal mol ${ }^{-1}$. Atom colors: Black $-\mathrm{C}$, Pink $-\mathrm{S}$, Red $-\mathrm{O}$. Energies refer to $\Delta \mathrm{E}$ in $\mathrm{CH}_{3} \mathrm{CN}$ computed at the PCM/B3LYP/6-311+G**//B3LYP/6-31G ${ }^{*}$ level, Energies are reported with respect to isolated reactants]

Optimized geometries of transoid TSs reveals that both electronic and steric factors play an equally important role in imparting unexpectedly high energy for this particular TS. While unfavorable interactions between COMe (enone) and COPh (Ylide) substituents destabilize TS-4t, absence of such interactions in the corresponding syn TS (TS-4't) results in larger energy separation between the two pathways. Additionally, the latter TS is stabilized by better non-classical $\mathrm{H}$-bond interactions involving enone oxygen, which are relatively weaker in the former. 


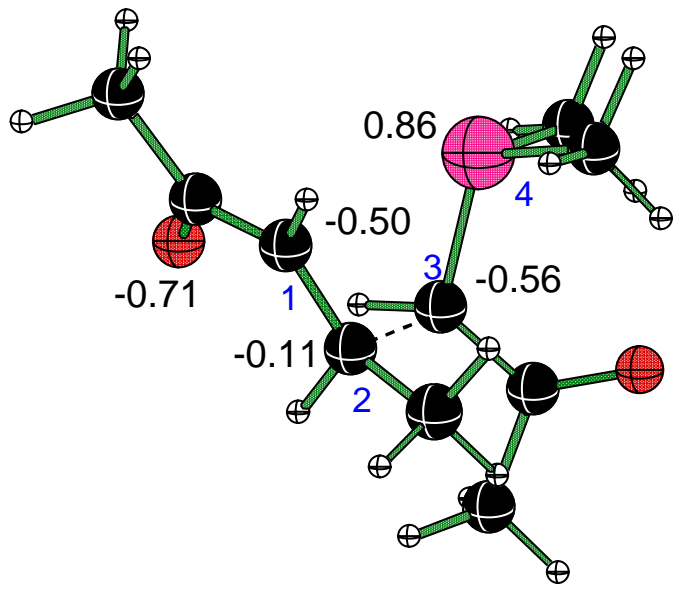

TS-5 $c$

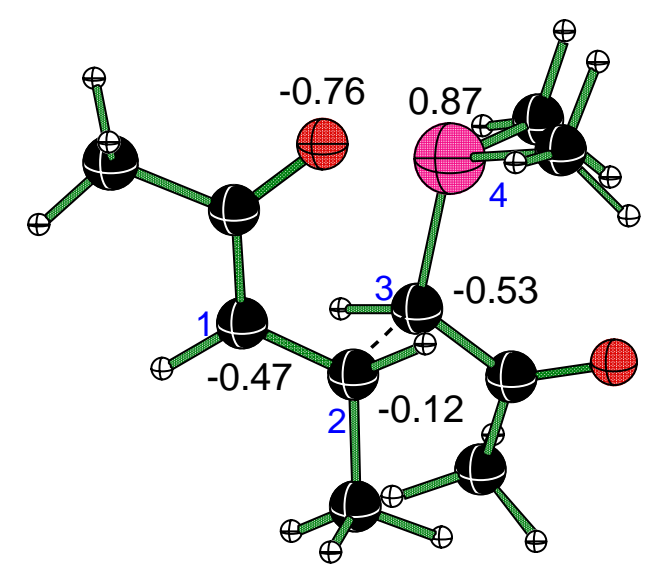

$\mathrm{TS}-\mathbf{5}^{\prime} c$

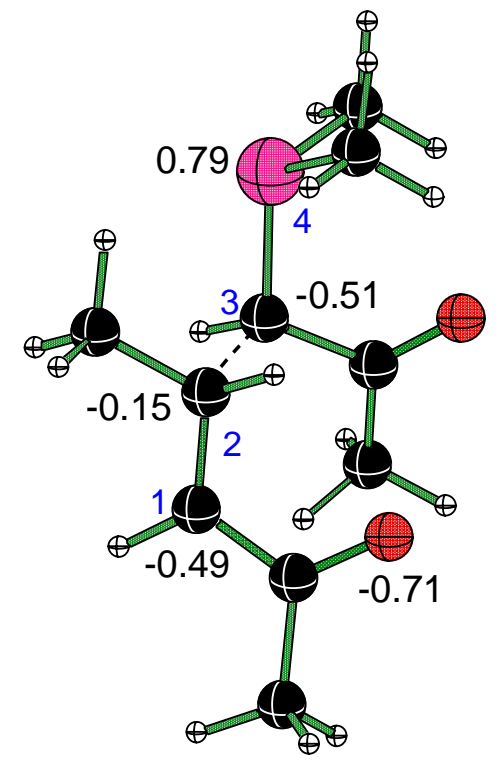

TS-5 $t$

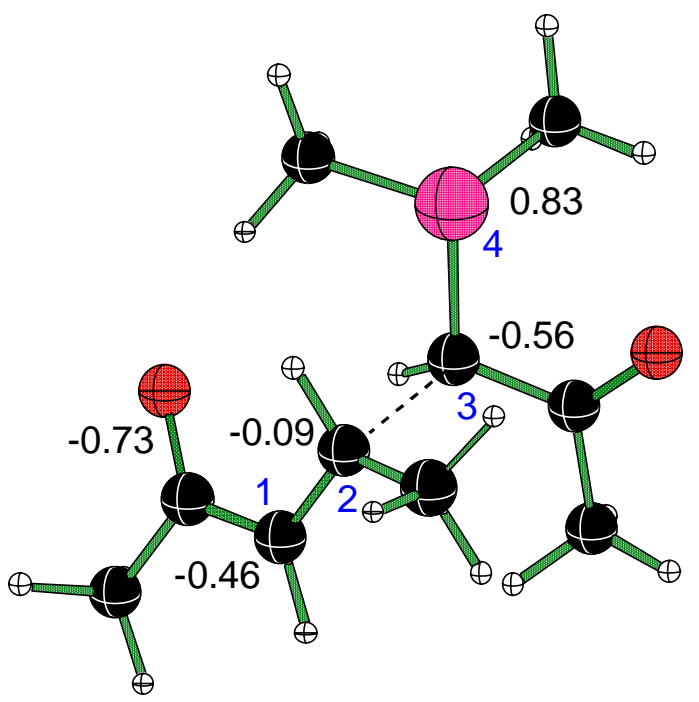

TS-5't

Figure S6. NPA charges computed at the B3LYP/6-311+G**//B3LYP/6-31G* level on developing charge centers for different addition transition states for ylide 5 . 


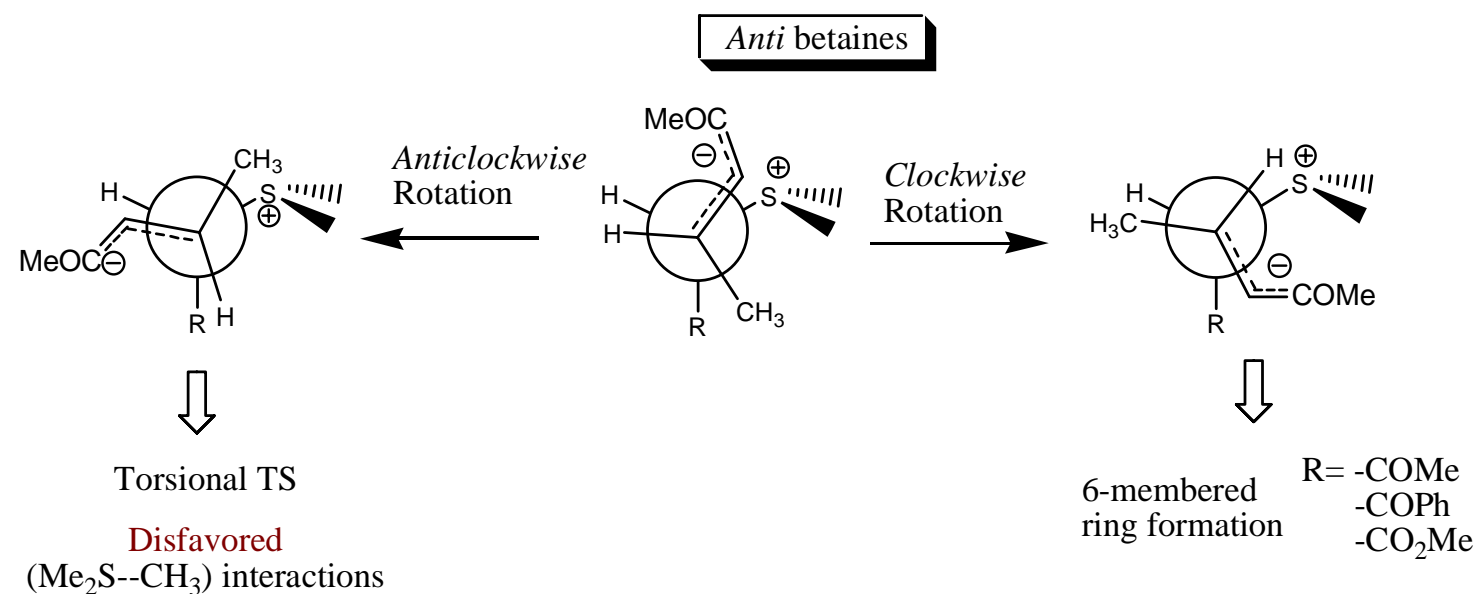

\section{Syn betaines}

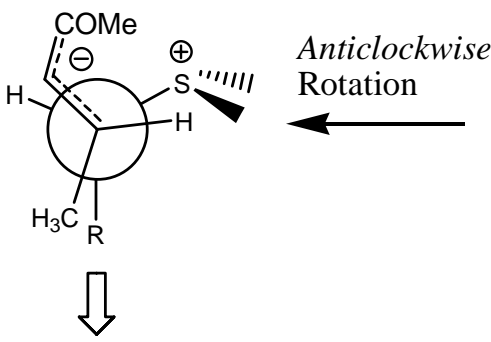

Torsional TS

Favored

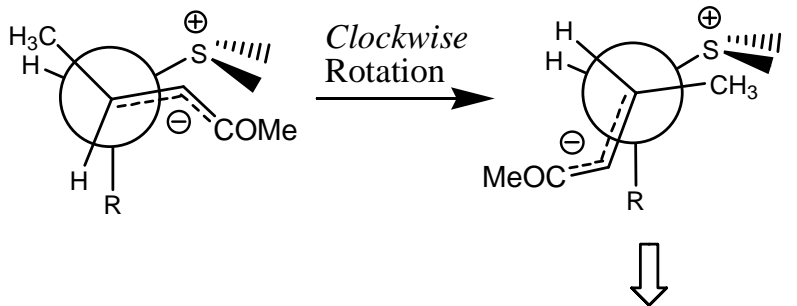

6-membered $\mathrm{R}=-\mathrm{COMe}$ $\begin{array}{ll}\text { ring formation } & -\mathrm{COPh} \\ & -\mathrm{CO}_{2} \mathrm{Me}\end{array}$

Scheme S1. Torsional rotation possibilities for syn and anti betaines of stabilized ylides. 
Table S1. Relative energies of intermediates and products (in $\mathrm{kcal} \mathrm{mol}^{-1}$ ) computed at the PCM/B3LYP/6-311+G**//B3LYP/6-31G ${ }^{*}$ level of theory for the formation of cyclopropanes using acetonitrile solvent (energies with respect to the separated free reactants)

\begin{tabular}{cccc}
\hline Ylide & I-n $c^{\mathrm{a}}$ & $\mathrm{I}-\mathbf{n} t^{\mathrm{b}}$ & $\mathrm{PC}-\mathbf{n}^{\mathrm{c}}$ \\
\hline $\mathbf{1}^{\mathrm{d}}$ & -17.0 & -14.1 & -50.6 \\
$\mathbf{2}$ & -7.1 & -2.2 & -39.3 \\
$\mathbf{2}^{\prime}$ & -9.0 & $-3.9^{\mathrm{e}}$ & -41.9 \\
$\mathbf{3}$ & 9.6 & 17.2 & -25.6 \\
$\mathbf{3}^{\prime}$ & 7.8 & 12.1 & -28.0 \\
$\mathbf{4}$ & 16.5 & 21.7 & -18.5 \\
$\mathbf{4}^{\prime}$ & 15.9 & 18.3 & -22.3 \\
$\mathbf{5}$ & 13.6 & 17.0 & -22.1 \\
$\mathbf{5}^{\prime}$ & 12.8 & 13.3 & -22.5 \\
${ }^{\mathrm{a}}$ Cisoid betaine. ${ }^{\mathrm{b}}$ Transoid betaine. ${ }^{\mathrm{c}}$ Product $\mathrm{Complex}$ \\
${ }^{\mathrm{d}}$ Ylide $\mathbf{1}$ lacks prochiral faces. \\
${ }^{\mathrm{e}}$ Energy at the PCM $\left(\mathrm{CH}_{3} \mathrm{CN}\right) / \mathrm{B} 3 \mathrm{LYP} / 6-311+\mathrm{G}^{* *} / / \mathrm{PCM}\left(\mathrm{CH}_{3} \mathrm{CN}\right) / \mathrm{B} 3 \mathrm{LYP} /$ \\
\\
6-31G ${ }^{*}$ level.
\end{tabular}

Table S2. Acctivation barriers for addition TSs (high energy conformers) (in $\mathrm{kcal} \mathrm{mol}^{-1}$ ) computed at the PCM/B3LYP/6-311+G $\mathrm{G}^{* *} / / \mathrm{B} 3 \mathrm{LYP} / 6-31 \mathrm{G}^{*}$ level of theory for the formation of cyclopropanes using acetonitrile solvent (energies with respect to the separated free reactants)

\begin{tabular}{ccc}
\hline Ylide & TS-n $c^{\mathrm{a}}$ & TS-n $t^{\mathrm{b}}$ \\
\hline $\mathbf{2}$ & 13.8 & 19.1 \\
$\mathbf{2}^{\prime}$ & 11.9 & 13.5 \\
$\mathbf{3}$ & 19.7 & 24.3 \\
$\mathbf{3}^{\prime}$ & 21.6 & 21.5 \\
$\mathbf{4}$ & 24.1 & 32.2 \\
$\mathbf{4}^{\prime}$ & 24.3 & 26.8 \\
$\mathbf{5}$ & 21.3 & 26.3 \\
$\mathbf{5}^{\prime}$ & 23.3 & 22.8 \\
${ }^{\mathrm{a}}$ Cisoid addition. & ${ }^{\mathrm{b}}$ Transoid addition.
\end{tabular}


Table S3. Comparison of energies for various stationary points along the anti pathway for ylide $\mathbf{3}$ (in kcal mol $\left.{ }^{-1}\right)^{\mathrm{a}}$

\begin{tabular}{ccc}
\hline Stationary Points & $\mathrm{E}_{\text {Rel }}(\mathrm{B} 3 \mathrm{LYP})^{\mathrm{b}}$ & $\mathrm{E}_{\text {Rel }}(\mathrm{HF})^{\mathrm{c}}$ \\
\hline $\mathrm{TS}-3 c$ & 17.8 & 18.9 \\
$\mathrm{TS}-3 t$ & 21.4 & 21.3 \\
$\mathrm{TS}-3 \mathrm{R}$ & $-{ }^{\mathrm{d}}$ & 19.6 \\
$\mathrm{TS}-3 \mathrm{E}$ & 19.4 & 16.2 \\
$\mathrm{I}-3 \mathrm{c} c$ & 9.6 & 8.7 \\
$\mathrm{I}-3 t$ & 17.2 & 15.5 \\
$\mathrm{PC}-3$ & -25.6 & -26.7 \\
\hline${ }^{\mathrm{a}}$ Energies relative to separated reactants & \\
${ }^{\mathrm{b}} \mathrm{PCM}\left(\mathrm{CH}_{3} \mathrm{CN}\right) / \mathrm{B} 3 \mathrm{LYP} / 6-311+\mathrm{G}^{* *} / / \mathrm{B} 3 \mathrm{LYP} / 6-31 \mathrm{G}^{*}$ level \\
${ }^{\mathrm{c}} \mathrm{PCM}\left(\mathrm{CH}_{3} \mathrm{CN}\right) / \mathrm{B} 3 \mathrm{LYP} / 6-311+\mathrm{G}^{* *} / / \mathrm{HF} / 6-31 \mathrm{G}^{*}$ level \\
${ }^{\mathrm{d}}$ Torsional TS could not be located at the B3LYP/6-31G ${ }^{*}$ level
\end{tabular}


Table S4. IRC profiles for addition and elimination TSs for sulfur ylide mediated cyclporopanation reaction.

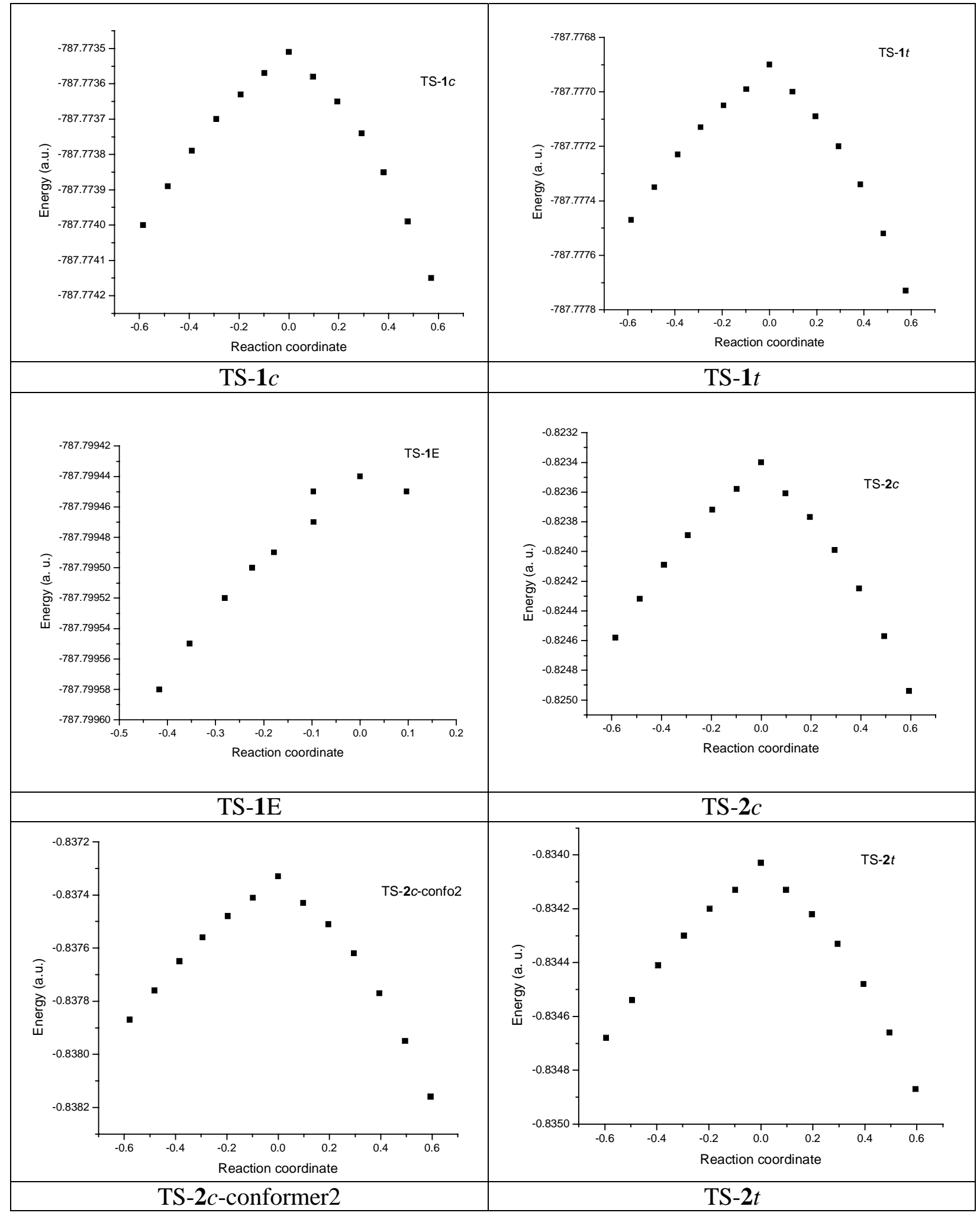




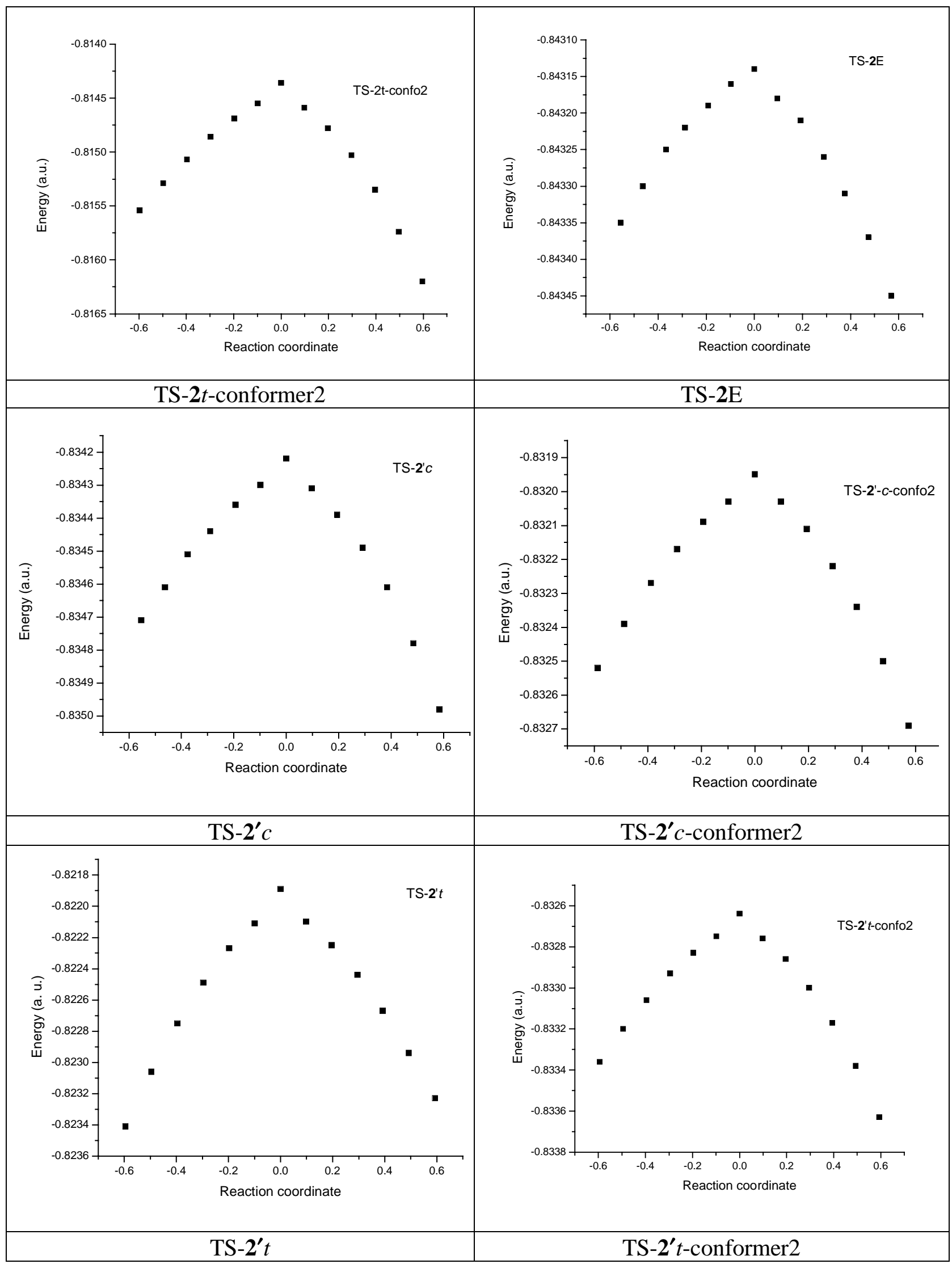




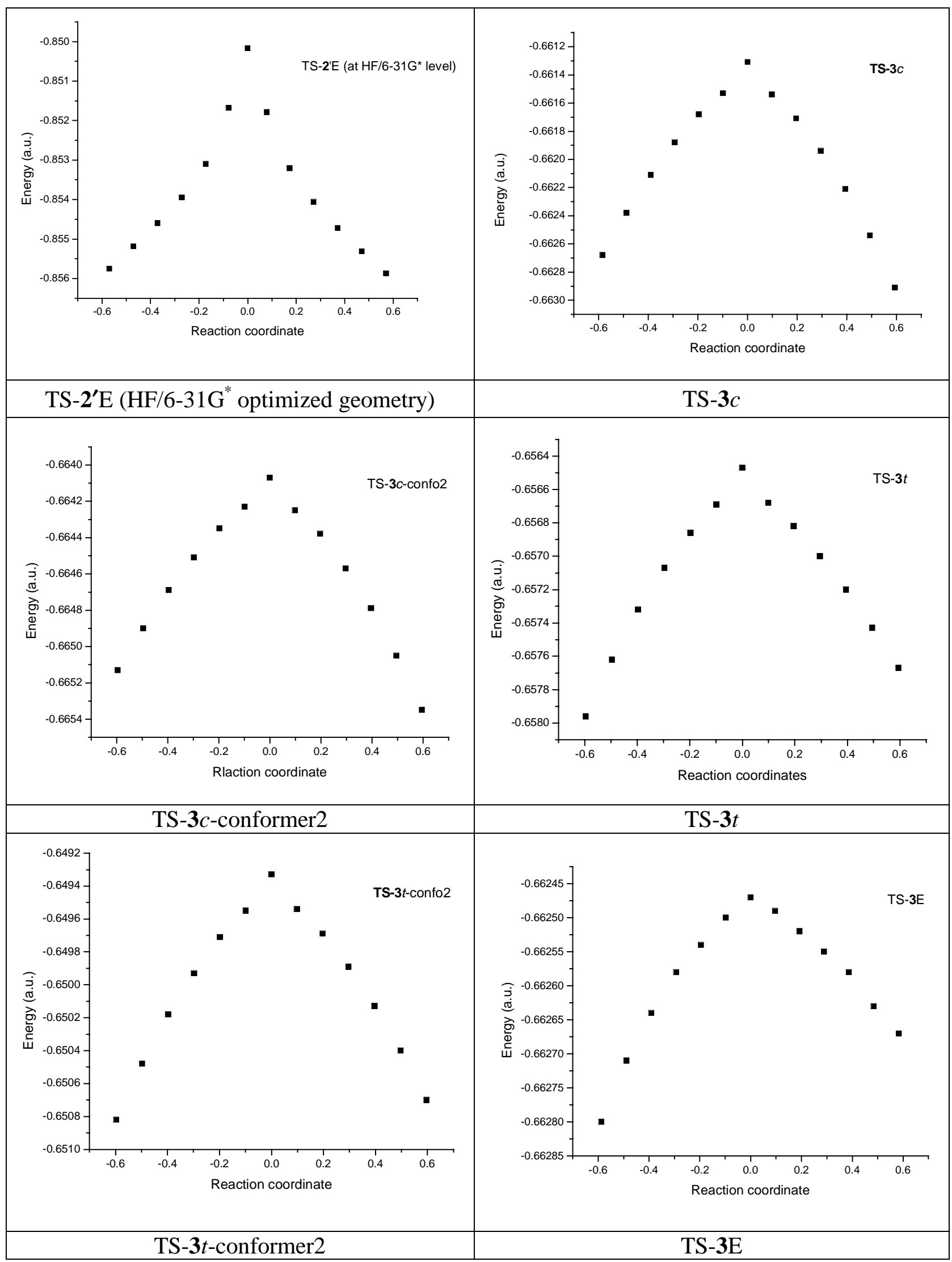




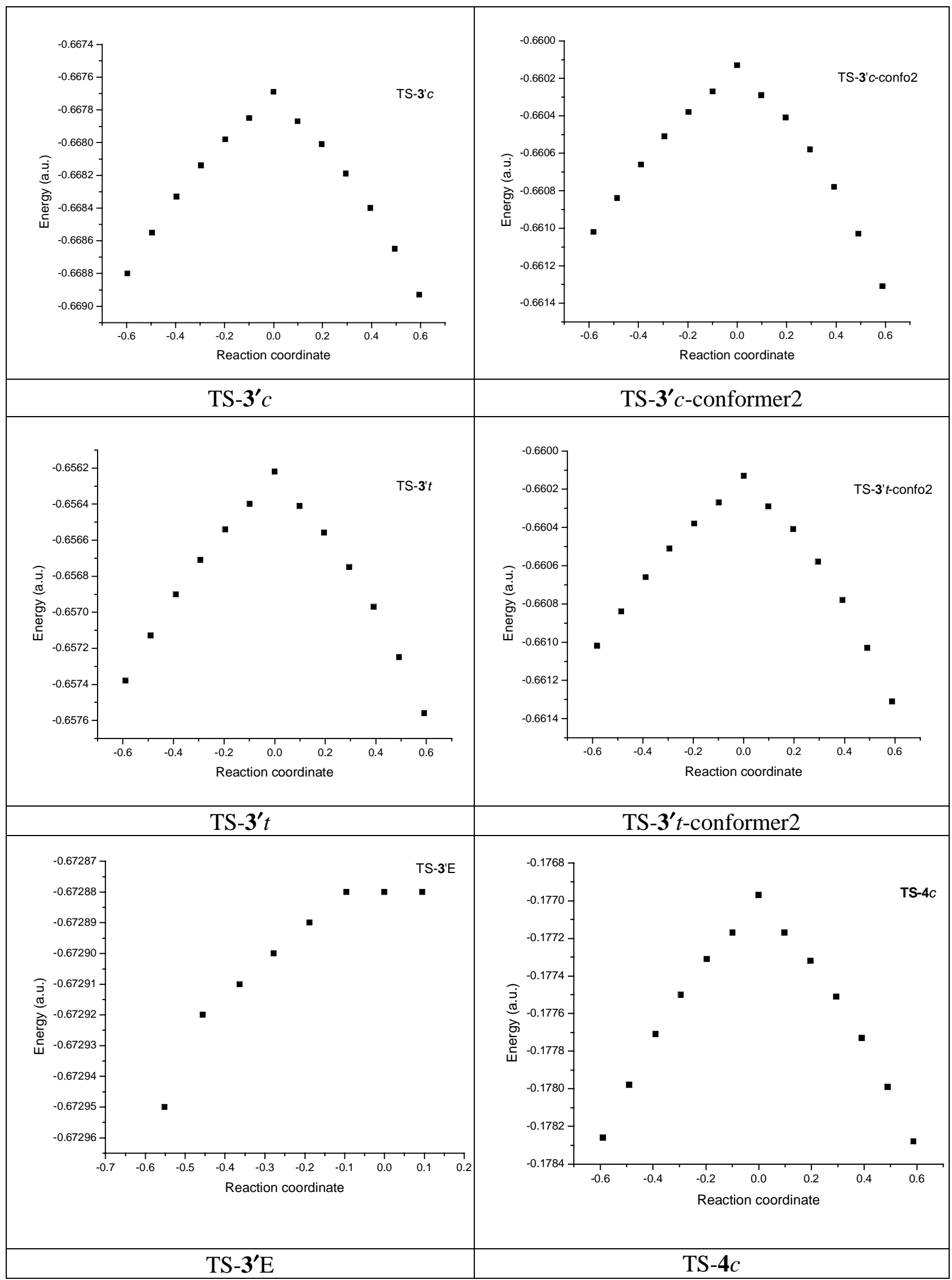




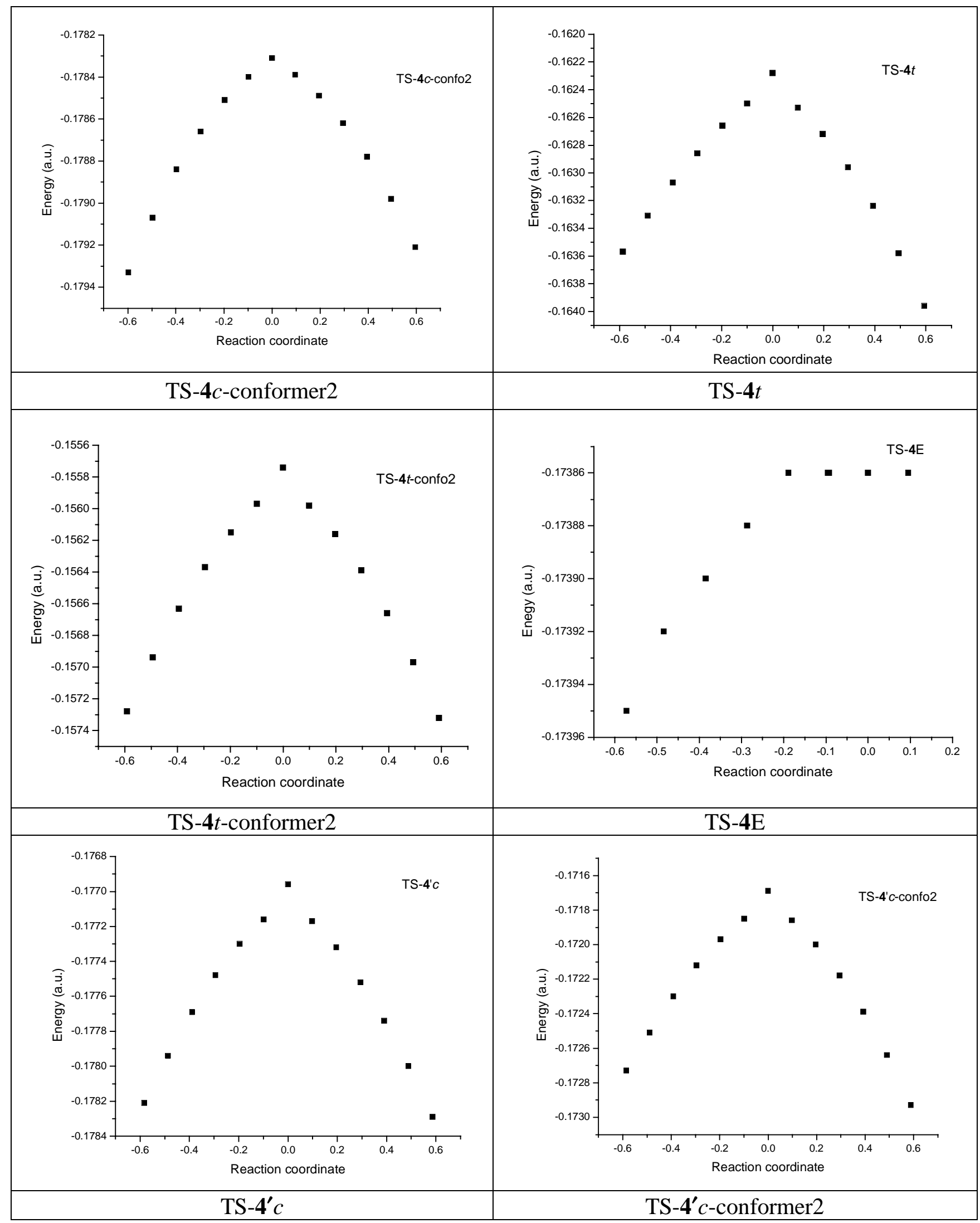




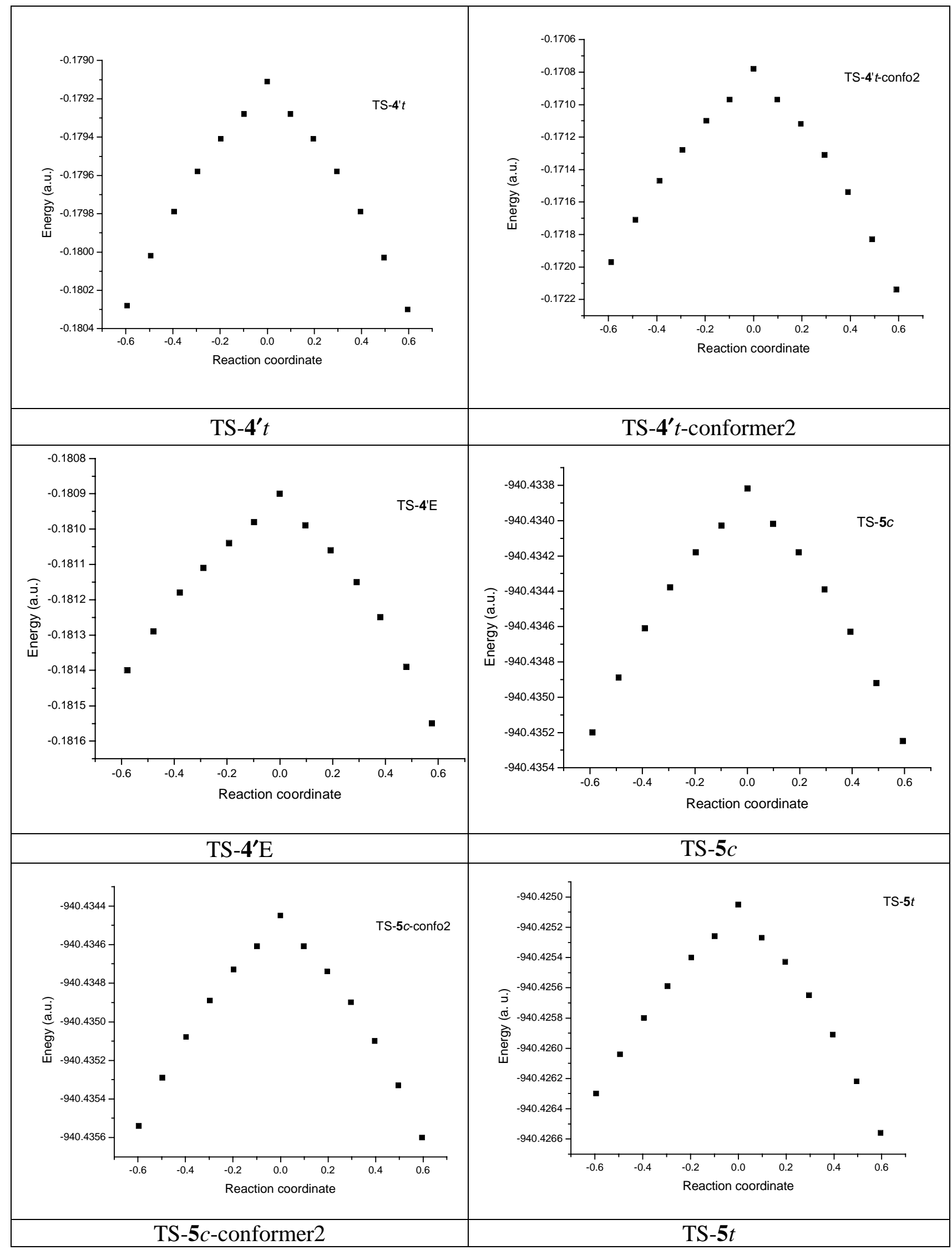




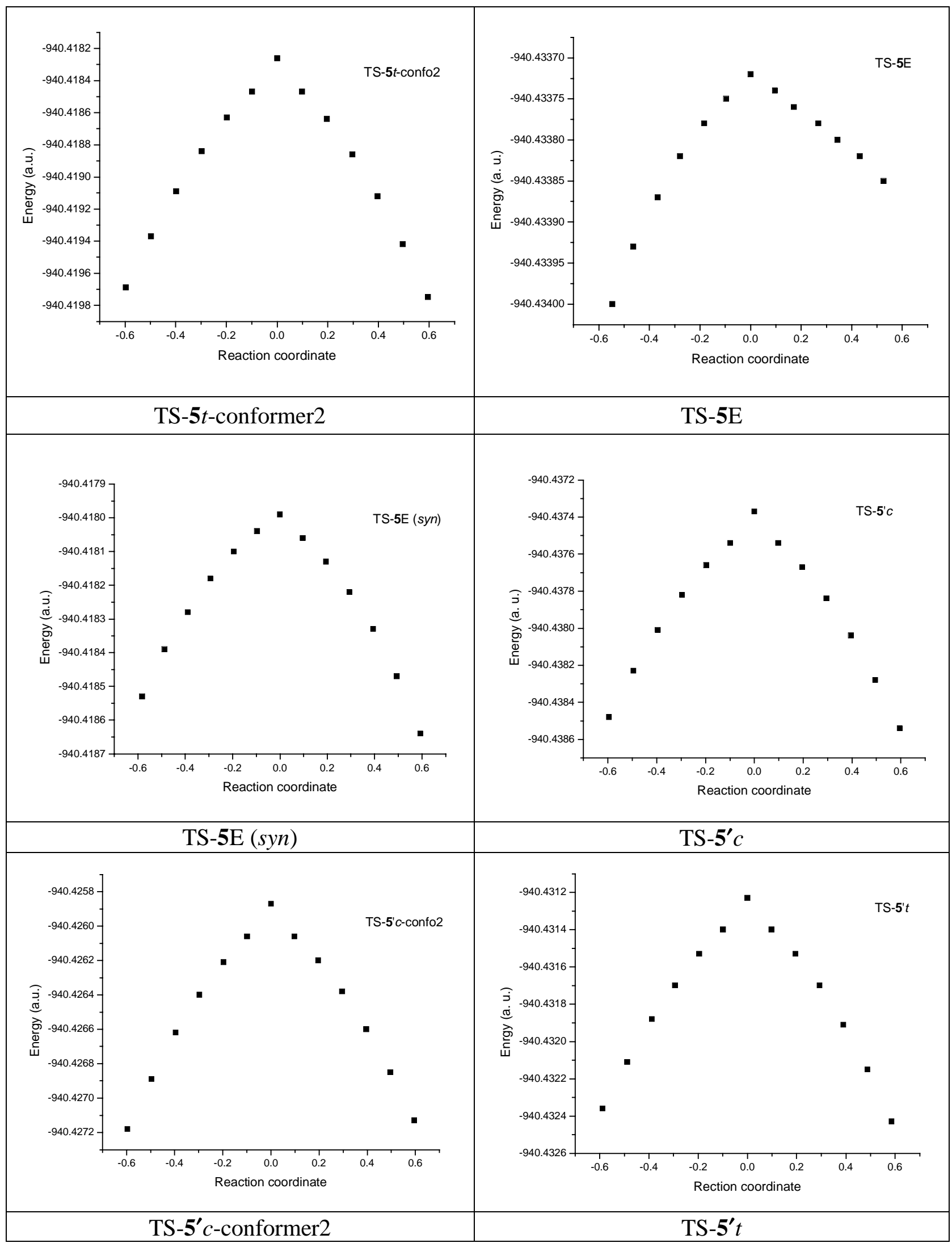




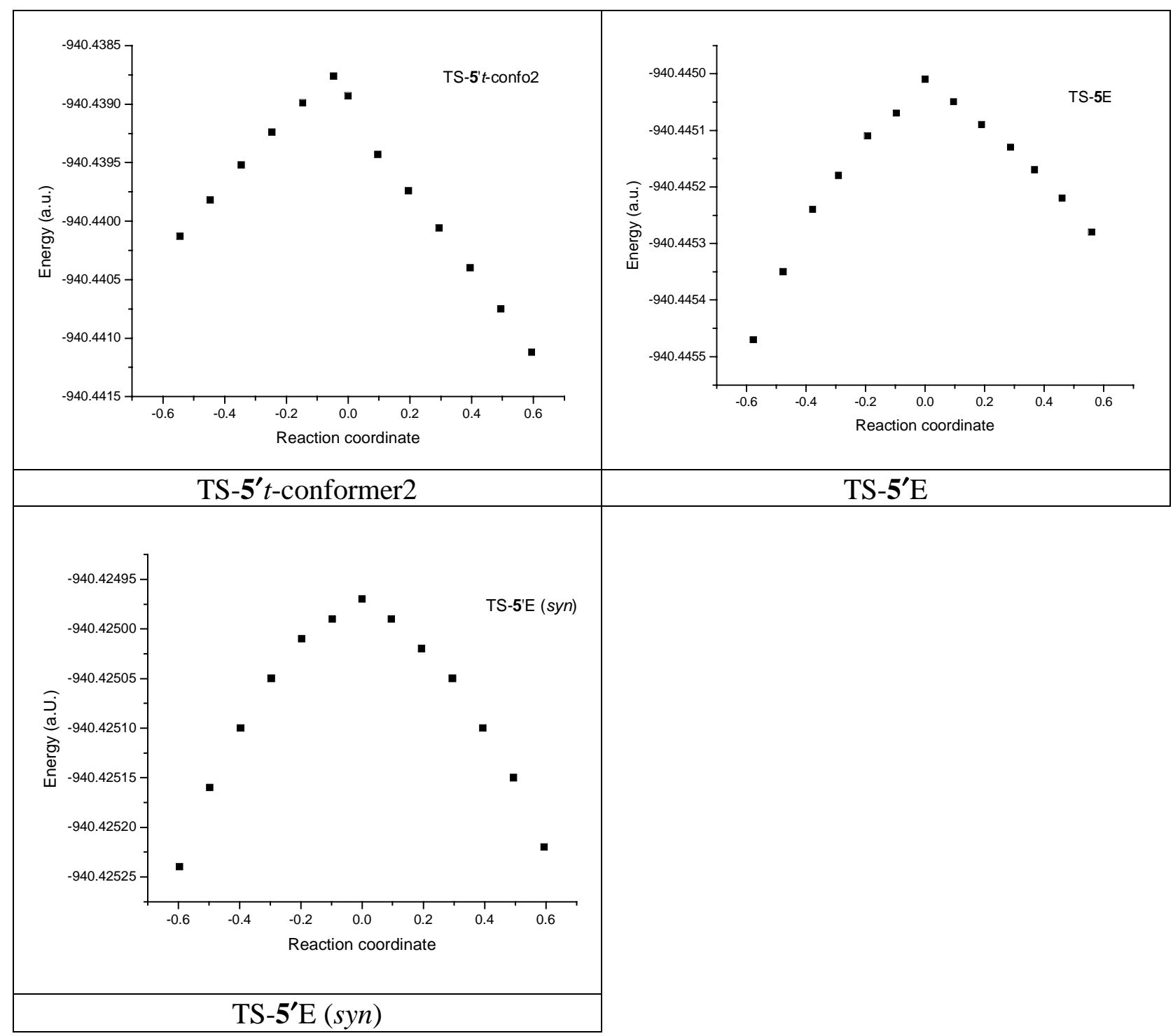

Full list of citations for Gaussian98 and Gaussian03 (Reference 17 in the text)

\section{Gaussian98:}

Gaussian 98, Revision A.11.4, Frisch, M. J.; Trucks, G. W.; Schlegel, H. B.; Scuseria, G.

E.; Robb, M. A.; Cheeseman, J. R.; Zakrzewski, V. G.; Montgomery, J. A., Jr.; Stratmann, R. E.; Burant, J. C.; Dapprich, S.; Millam, J. M.; Daniels, A.D.; Kudin, K. N.; Strain, M. C.; Farkas, O.; Tomasi, J.; Barone, V.; Cossi, M.; Cammi, R.; Mennucci, B.; Pomelli, C.; Adamo, C.; Clifford, S.; Ochterski, J. W.; Petersson, G. A.; Ayala, P. Y.; Cui, Q.; Morokuma, K.; Malick, D. K.; Rabuck, A. D.; Raghavachari, K.; Foresman, J. 
B.; Cioslowski, J.; Ortiz, J. V.; Stefanov, B. B.; Liu, G.; Liashenko, A.; Piskorz, P.;

Komaromi, I.; Gomperts, R.; Martin, R. L.; Fox, D. J.; Keith, T.; Al-Laham, M. A.; Peng,

C. Y.; Nanayakkara, A.; Gonzalez, C.; Challacombe, M.; Gill, P. M. W.; Johnson, B.;

Chen, W.; Wong, M. W.; Andres, J. L.; Head-Gordon, M.; Replogle, E. S.; Pople, J. A., Gaussian, Inc., Pittsburgh PA, 2002.

Gaussian03:

Gaussian 03, Revision C.02, Frisch, M. J.; Trucks, G. W.; Schlegel, H. B.; Scuseria, G.

E.; Robb, M. A.; Cheeseman, J. R.; Montgomery, Jr., J. A.; Vreven, T.; Kudin, K. N.;

Burant, J. C.; Millam, J. M.; Iyengar, S. S.; Tomasi, J.; Barone, V.; Mennucci, B.; Cossi, M.; Scalmani, G.; Rega, N.; Petersson, G. A.; Nakatsuji, H.; Hada, M.; Ehara, M.; Toyota, K.; Fukuda, R.; Hasegawa, J.; Ishida, M.; Nakajima, T.; Honda, Y.; Kitao, O.; Nakai, H.; Klene, M.; Li, X.; Knox, J. E.; Hratchian, H. P.; Cross, J. B.; Bakken, V.; Adamo, C.; Jaramillo, J.; Gomperts, R.; Stratmann, R. E.; Yazyev, O.; Austin, A. J.; Cammi, R.; Pomelli, C.; Ochterski, J. W.; Ayala, P. Y.; Morokuma, K.; Voth, G. A.; Salvador, P.; Dannenberg, J. J.; Zakrzewski, V. G.; Dapprich, S.; Daniels, A. D.; Strain, M. C.; Farkas, O.; Malick, D. K.; Rabuck, A. D.; Raghavachari, K.; Foresman, J. B.; Ortiz, J. V.; Cui, Q.; Baboul, A. G.; Clifford, S.; Cioslowski, J.; Stefanov, B. B.; Liu, G.; Liashenko, A.; Piskorz, P.; Komaromi, I.; Martin, R. L.; Fox, D. J.; Keith, T.; Al-Laham, M. A.; Peng, C. Y.; Nanayakkara, A.; Challacombe, M.; Gill, P. M. W.; Johnson, B.; Chen, W.; Wong, M. W.; Gonzalez, C.; Pople, J. A., Gaussian, Inc., Wallingford CT, 2004. 
Optimized Cartesian coordinates for transition states, intermediates, product complexes, substituted ylides and enone $(\mathrm{MeCH}=\mathrm{CH}-\mathrm{COMe})$ for sulfur ylide mediated cylopropanation reaction.

$E_{t}$ in a.u. (B3LYP/6-31G ${ }^{*}$ ). For TS-3R and TS-4R, coordinates are provided for the mPW1PW91/6-31G ${ }^{*}$ optimized geometry. For transition states TS-2R and TS-2E, geometries optimized at the $\mathrm{HF} / 6-31 \mathrm{G}^{*}$ level is given [Single-point energy computed at the $\mathrm{PCM}\left(\mathrm{CH}_{3} \mathrm{CN}\right) / \mathrm{B} 3 \mathrm{LYP} / 6-311+\mathrm{G}^{* *} / / \mathrm{B} 3 \mathrm{LYP} / 6-31 \mathrm{G}^{*}$ level, Number of imaginary frequencies (NImag, values in $\mathrm{cm}^{-1}$ ), if any, are given after the respective geometries]. The HF/6-31G ${ }^{*}$ coordinates and energies (a.u.) for all the stationary points computed for ylide 3 ( $R e, S i$ pathway), and solvent optimized coordinates and energies at the $\mathrm{PCM}\left(\mathrm{CH}_{3} \mathrm{CN}\right) / \mathrm{B} 3 \mathrm{LYP} / 6-31 \mathrm{G}^{*}$ for representative stabilized ylide 5 (anti and syn pathways) are also provided (Please see computational details for further information regarding these calculations).

1. TS-1 $c(-787.77351)$

$\begin{array}{cccc}6 & -3.546884 & -0.534155 & -0.010441 \\ 1 & -3.793464 & -0.108557 & 0.966378 \\ 1 & -4.087801 & -0.005942 & -0.798967 \\ 16 & -1.725001 & -0.329140 & -0.361303 \\ 6 & -1.109587 & -1.233741 & 1.092863 \\ 1 & -1.641323 & -0.900202 & 1.988133 \\ 1 & -0.047044 & -1.002184 & 1.171972 \\ 6 & -1.177258 & 1.287554 & -0.043160 \\ 1 & -1.454071 & 1.924707 & -0.882932 \\ 6 & 1.012585 & 1.462539 & -0.237569 \\ 6 & 1.093219 & 2.901372 & 0.217766 \\ 1 & 1.087499 & 2.977465 & 1.311034 \\ 1 & 2.035510 & 3.342867 & -0.135267 \\ 1 & 0.276184 & 3.516249 & -0.173203 \\ 1 & 0.852713 & 1.307691 & -1.300764 \\ 6 & 1.794235 & 0.488173 & 0.380423 \\ 1 & 2.268339 & 0.694746 & 1.338152 \\ 6 & 1.971435 & -0.790995 & -0.241159 \\ 6 & 3.033934 & -1.734622 & 0.324146 \\ 1 & 3.489683 & -1.367631 & 1.249856 \\ 1 & 2.596547 & -2.723937 & 0.501937 \\ 1 & 3.826219 & -1.868886 & -0.422423 \\ 8 & 1.292279 & -1.171298 & -1.217150 \\ 1 & -1.258981 & -2.302335 & 0.927656 \\ 1 & -3.798533 & -1.599656 & -0.040615 \\ 1 & -1.519769 & 1.675488 & 0.919890 \\ & & & \\ \mathrm{E}(\mathrm{MeCN})=-787.9258546 & & \\ \mathrm{NImag}=1(-241.6) & & \end{array}$

2. I-1c (-787.81144)

$\begin{array}{lrrr}6 & -3.172501 & -0.024263 & 0.032201 \\ 1 & -3.260216 & 0.388771 & 1.041981 \\ 1 & -3.551073 & 0.696470 & -0.698009 \\ 16 & -1.399252 & -0.438975 & -0.433988 \\ 6 & -1.099484 & -1.580567 & 0.965248 \\ 1 & -1.965275 & -1.601540 & 1.631107 \\ 1 & -0.209480 & -1.219978 & 1.483996 \\ 6 & -0.735012 & 1.195886 & 0.149710 \\ 1 & -1.436491 & 1.915549 & -0.289846 \\ 6 & 0.712273 & 1.515452 & -0.252356 \\ 6 & 1.012243 & 2.955358 & 0.194902 \\ 1 & 0.972035 & 3.047320 & 1.287920 \\ 1 & 2.022205 & 3.236236 & -0.122227 \\ 1 & 0.308746 & 3.679861 & -0.234349 \\ 1 & 0.731566 & 1.486370 & -1.354820 \\ 6 & 1.721265 & 0.531390 & 0.283186 \\ 1 & 2.540461 & 0.892930 & 0.897936 \\ 6 & 1.674138 & -0.753441 & -0.172510 \\ 6 & 2.744189 & -1.771572 & 0.176690 \\ 1 & 3.532423 & -1.351029 & 0.810409 \\ 1 & 2.302071 & -2.636745 & 0.688632 \\ 1 & 3.201963 & -2.154122 & -0.744136 \\ 8 & 0.696096 & -1.183444 & -0.930236 \\ 1 & -0.894234 & -2.557341 & 0.531771 \\ 1 & -3.757139 & -0.945462 & -0.045596 \\ 1 & -0.860958 & 1.204411 & 1.238504 \\ \mathrm{E}(\mathrm{MeCN})=-787.9633193 & & \\ \mathrm{NImag}=0 & & & \\ & & & \end{array}$


3. TS-1 $t(-787.77690)$

$\begin{array}{cccc}6 & 3.169307 & -1.145316 & -.886688 \\ 1 & 2.486322 & -1.975373 & -1.085312 \\ 1 & 3.536089 & -.724165 & -1.824866 \\ 16 & 2.251414 & .212523 & -.004814 \\ 6 & 1.624060 & -.791403 & 1.395955 \\ 1 & .675022 & -1.262152 & 1.107456 \\ 1 & 1.448048 & -.108714 & 2.229777 \\ 6 & .786817 & .675751 & -.837076 \\ 1 & 1.021901 & 1.478271 & -1.537491 \\ 6 & -.937851 & 1.298167 & .303396 \\ 6 & -.828614 & 2.802049 & .217517 \\ 1 & -1.089644 & 3.168197 & -.781960 \\ 1 & -1.527638 & 3.264977 & .927862 \\ 1 & .178557 & 3.156128 & .459733 \\ 1 & -.542208 & .856683 & 1.212420 \\ 6 & -2.020671 & .619262 & -.250636 \\ 1 & -2.647819 & 1.109821 & -.992222 \\ 6 & -2.227044 & -.768606 & .031565 \\ 6 & -3.470345 & -1.446604 & -.541128 \\ 1 & -4.121450 & -.759280 & -1.090700 \\ 1 & -3.170546 & -2.263114 & -1.209484 \\ 1 & -4.041971 & -1.900479 & .276600 \\ 8 & -1.439358 & -1.465098 & .710364 \\ 1 & 2.390886 & -1.517218 & 1.676666 \\ 1 & 4.011316 & -1.476731 & -.271350 \\ 1 & .311415 & -.186248 & -1.300455\end{array}$

$\mathrm{E}(\mathrm{MeCN})=-787.9279418$

NImag $=1(-257.7)$

\section{I-1 $t(-787.79774)$}

$\begin{array}{crrr}6 & 2.762351 & -1.037005 & -1.103704 \\ 1 & 2.024831 & -1.816698 & -1.312348 \\ 1 & 2.998275 & -0.481164 & -2.013772 \\ 16 & 2.061776 & 0.135191 & 0.102849 \\ 6 & 1.507708 & -1.026202 & 1.414314 \\ 1 & 0.477867 & -1.344960 & 1.138243 \\ 1 & 1.523242 & -0.479976 & 2.358302 \\ 6 & 0.486365 & 0.547013 & -0.695511 \\ 1 & 0.741481 & 1.157257 & -1.568956 \\ 6 & -0.504549 & 1.266816 & 0.264388 \\ 6 & -0.399410 & 2.790091 & 0.131515 \\ 1 & -0.673589 & 3.109414 & -0.881847 \\ 1 & -1.089024 & 3.277502 & 0.828868 \\ 1 & 0.612721 & 3.160433 & 0.344484 \\ 1 & -0.217832 & 1.006546 & 1.301599 \\ 6 & -1.868723 & 0.724960 & -0.057228 \\ 1 & -2.652574 & 1.424213 & -0.332276 \\ 6 & -2.098105 & -0.636388 & -0.001029 \\ 6 & -3.481551 & -1.173215 & -0.352491 \\ 1 & -4.194210 & -0.383682 & -0.616539\end{array}$

$\begin{array}{lrrr}1 & -3.405229 & -1.876521 & -1.192152 \\ 1 & -3.883842 & -1.741557 & 0.495922 \\ 8 & -1.205483 & -1.521769 & 0.301391 \\ 1 & 2.227561 & -1.847595 & 1.449887 \\ 1 & 3.676094 & -1.465898 & -0.686624 \\ 1 & 0.065164 & -0.422639 & -0.971013\end{array}$

$\mathrm{E}(\mathrm{MeCN})=-787.9588203$

NImag $=0$

5. TS-1R (-787.79631)

$\begin{array}{lccc}6 & -3.344997 & -0.010323 & 0.612774 \\ 1 & -3.136486 & -0.297473 & 1.646596 \\ 1 & -3.636449 & 1.041589 & 0.565544 \\ 16 & -1.856992 & -0.228177 & -0.421701 \\ 6 & -1.435358 & -1.975394 & -0.139955 \\ 1 & -1.782614 & -2.277515 & 0.851648 \\ 1 & -0.324080 & -1.991559 & -0.222081 \\ 6 & -0.518656 & 0.660396 & 0.608436 \\ 1 & -1.071943 & 1.386361 & 1.218713 \\ 6 & 0.575061 & 1.305907 & -0.288877 \\ 6 & 0.441482 & 2.834034 & -0.287443 \\ 1 & 0.605116 & 3.243468 & 0.718025 \\ 1 & 1.190194 & 3.278307 & -0.951119 \\ 1 & -0.548857 & 3.164802 & -0.629917 \\ 1 & 0.404478 & 0.942067 & -1.317114 \\ 6 & 1.899176 & 0.800726 & 0.197140 \\ 1 & 2.616509 & 1.483953 & 0.641339 \\ 6 & 2.159620 & -0.539829 & -0.007827 \\ 6 & 3.492723 & -1.140890 & 0.419473 \\ 1 & 4.164224 & -0.405674 & 0.877382 \\ 1 & 3.326575 & -1.958568 & 1.133502 \\ 1 & 3.994937 & -1.582298 & -0.450751 \\ 8 & 1.308417 & -1.331931 & -0.567169 \\ 1 & -1.911501 & -2.571339 & -0.920749 \\ 1 & -4.148468 & -0.627874 & 0.204773 \\ 1 & -0.111413 & -0.129729 & 1.236455 \\ & & & \\ \mathrm{E}(\mathrm{MeCN})=-787.9576564 & & \\ \mathrm{NImag}=1(-74.6) & & \\ & & & \end{array}$

6. TS-1E (-787.79944)

$\begin{array}{crrr}6 & 2.180884 & -1.569192 & -0.920898 \\ 16 & 2.194699 & 0.097550 & -0.178861 \\ 6 & 0.353534 & 0.640359 & -0.845379 \\ 6 & -0.561788 & 1.330551 & 0.173908 \\ 6 & -1.875405 & 0.670153 & -0.130915 \\ 6 & -1.988379 & -0.692904 & 0.130450 \\ 8 & -1.027828 & -1.403881 & 0.600805 \\ 6 & 1.791786 & -0.387195 & 1.538554 \\ 6 & -0.556413 & 2.854185 & 0.020298\end{array}$




\begin{tabular}{|c|c|c|c|c|c|c|c|}
\hline 6 & -3.296875 & -1.410980 & -0.178853 & \multicolumn{4}{|c|}{ 8. TS-2c $(-1018.82340)$} \\
\hline 1 & 1.221965 & -2.035162 & -0.664565 & & & & \\
\hline 1 & 2.287663 & -1.460058 & -2.002445 & 6 & 0.624639 & 2.605077 & -1.431452 \\
\hline 1 & 0.790718 & -0.842291 & 1.516960 & 1 & 1.624521 & 2.481381 & -1.011074 \\
\hline 1 & 1.816249 & 0.510814 & 2.157615 & 1 & 0.606416 & 2.237700 & -2.459415 \\
\hline 1 & 0.588518 & 1.245826 & -1.726164 & 16 & -0.597809 & 1.615464 & -0.499521 \\
\hline 1 & -0.893356 & 3.148895 & -0.981555 & 6 & -0.568950 & 2.482283 & 1.110321 \\
\hline 1 & -1.237913 & 3.310092 & 0.746090 & 1 & 0.426522 & 2.502743 & 1.553573 \\
\hline 1 & 0.442447 & 3.282040 & 0.179151 & 1 & -1.267797 & 1.945626 & 1.751511 \\
\hline 1 & -0.243014 & 1.081978 & 1.194697 & 6 & 0.039385 & -0.030920 & -0.305715 \\
\hline 1 & -2.671833 & 1.243129 & -0.595200 & 1 & -0.480324 & -0.570789 & -1.102287 \\
\hline 1 & -4.061391 & -0.746048 & -0.595717 & 6 & -1.119097 & -0.981337 & 1.059074 \\
\hline 1 & -3.114794 & -2.230045 & -0.886686 & 6 & -0.476199 & -0.816781 & 2.418895 \\
\hline 1 & -3.691016 & -1.869375 & 0.736712 & 1 & -0.817443 & 0.086726 & 2.938244 \\
\hline 1 & 2.566622 & -1.082605 & 1.872049 & 1 & -0.757274 & -1.669957 & 3.050428 \\
\hline 1 & 3.020326 & -2.144174 & -0.524227 & 1 & 0.616909 & -0.796522 & 2.363881 \\
\hline 1 & 0.002181 & -0.352622 & -1.094374 & 1 & -0.825702 & -1.905141 & 0.560126 \\
\hline & & & & 6 & -2.478468 & -0.633609 & 0.870148 \\
\hline $\mathrm{E}(\mathrm{M}$ & 787.9551046 & & & 1 & -2.997386 & -0.031339 & 1.614074 \\
\hline NIm & 48.6) & & & 6 & -3.126276 & -0.999382 & -0.342156 \\
\hline & & & & 6 & -4.618731 & -0.704719 & -0.495414 \\
\hline 7. $\mathrm{PC}$ & 88044) & & & 1 & -5.053538 & -0.206972 & 0.378365 \\
\hline & & & & 1 & -4.784553 & -0.081033 & -1.382251 \\
\hline 6 & -3.018935 & 1.129476 & -0.380111 & 1 & -5.155680 & -1.645415 & -0.666012 \\
\hline 16 & -2.956095 & -0.692826 & -0.512355 & 8 & -2.526885 & -1.544311 & -1.300336 \\
\hline 6 & 1.249075 & -0.955974 & -1.013102 & 1 & -0.933799 & 3.497590 & 0.934828 \\
\hline 6 & 2.070096 & -1.146224 & 0.213627 & 1 & 0.314044 & 3.653159 & -1.407541 \\
\hline 6 & 2.043942 & 0.227527 & -0.458747 & 6 & 1.483728 & -0.291796 & -0.235859 \\
\hline 6 & 1.290859 & 1.324015 & 0.207526 & 6 & 1.976968 & -1.424112 & -0.924757 \\
\hline 8 & 0.285071 & 1.110331 & 0.872992 & 6 & 3.313215 & -1.802310 & -0.836410 \\
\hline 6 & -2.464849 & -1.091806 & 1.202958 & 6 & 4.215290 & -1.055240 & -0.073422 \\
\hline 6 & 3.342845 & -1.964127 & 0.193196 & 6 & 3.751042 & 0.066520 & 0.615140 \\
\hline 6 & 1.830159 & 2.730951 & 0.025958 & 6 & 2.407883 & 0.435499 & 0.547272 \\
\hline 1 & -2.057269 & 1.520296 & -0.036917 & 1 & 1.290572 & -2.005601 & -1.535257 \\
\hline 1 & -3.236437 & 1.519500 & -1.378327 & 1 & 3.655921 & -2.680263 & -1.378373 \\
\hline 1 & -1.515200 & -0.607421 & 1.445422 & 1 & 5.260341 & -1.345240 & -0.013264 \\
\hline 1 & -2.347551 & -2.177521 & 1.261196 & 1 & 4.435086 & 0.654062 & 1.222793 \\
\hline 1 & 1.609711 & -1.380202 & -1.947082 & 1 & 2.076616 & 1.291298 & 1.128680 \\
\hline 1 & 3.860862 & -1.869468 & -0.768470 & & & & \\
\hline 1 & 4.036113 & -1.647371 & 0.981499 & E $(\mathrm{M}$ & 019.035503 & & \\
\hline 1 & 3.122887 & -3.027535 & 0.347489 & NIm & 0.7) & & \\
\hline 1 & 1.508678 & -1.173015 & 1.145539 & & & & \\
\hline 1 & 2.935936 & 0.521970 & -1.006564 & 9. $\mathrm{T}$ & rmer2 (-101 & $8.8373305)$ & \\
\hline 1 & 1.865341 & 2.990429 & -1.039808 & & & & \\
\hline 1 & 1.198502 & 3.445191 & 0.557643 & 6 & 2.391022 & 2.156643 & 0.224159 \\
\hline 1 & 2.858690 & 2.796246 & 0.403303 & 1 & 2.191902 & 2.578270 & 1.212975 \\
\hline 1 & -3.236855 & -0.782950 & 1.915061 & 1 & 2.960159 & 1.225416 & 0.265790 \\
\hline 1 & -3.813845 & 1.446724 & 0.302531 & 16 & 0.833230 & 1.699468 & -0.595833 \\
\hline 1 & 0.168228 & -0.895333 & -0.916586 & 6 & 0.119681 & 3.393425 & -0.813805 \\
\hline & & & & 1 & 0.085546 & 3.915111 & 0.146502 \\
\hline $\mathrm{E}(\mathrm{M}$ & 787.016962 & & & 1 & -0.890891 & 3.258583 & -1.204595 \\
\hline NIm & & & & 6 & -0.143376 & 0.903711 & 0.645961 \\
\hline & & & & 1 & -0.305863 & 1.593650 & 1.477786 \\
\hline & & & & 6 & 0.973377 & -0.711096 & 1.571298 \\
\hline
\end{tabular}




\begin{tabular}{|c|c|c|c|c|c|c|c|}
\hline 6 & 0.118609 & -0.872717 & 2.807473 & 1 & 0.691888 & -1.735371 & 3.269859 \\
\hline 1 & -0.858325 & -1.303900 & 2.563367 & 1 & 1.691028 & 0.025540 & 1.864953 \\
\hline 1 & 0.614768 & -1.555533 & 3.510137 & 1 & 1.038562 & -2.676633 & 0.792331 \\
\hline 1 & -0.046681 & 0.075263 & 3.330761 & 1 & 2.781299 & -2.243563 & -2.251645 \\
\hline 1 & 1.837770 & -0.065047 & 1.695177 & 1 & 4.050711 & -2.384518 & -1.040855 \\
\hline 6 & 1.145627 & -1.790635 & 0.700933 & 1 & 2.615972 & -3.433855 & -0.932236 \\
\hline 1 & 0.481271 & -2.648066 & 0.768577 & 6 & -2.560697 & 0.513070 & 0.682014 \\
\hline 6 & 2.108507 & -1.733982 & -0.344522 & 6 & -3.785336 & 0.049576 & 0.201533 \\
\hline 6 & 2.338550 & -2.975274 & -1.201284 & 6 & -3.818084 & -0.906386 & -0.815389 \\
\hline 1 & 1.691401 & -3.813634 & -0.925145 & 6 & -2.620733 & -1.394714 & -1.341045 \\
\hline 1 & 2.177851 & -2.730425 & -2.258076 & 6 & -1.394119 & -0.934020 & -0.860243 \\
\hline 1 & 3.384590 & -3.290547 & -1.105840 & 1 & -2.542859 & 1.254680 & 1.477777 \\
\hline 8 & 2.777122 & -0.704001 & -0.614478 & 1 & -4.710733 & 0.435722 & 0.620603 \\
\hline 1 & 0.726930 & 3.950368 & -1.533805 & 1 & -4.770164 & -1.266273 & -1.196328 \\
\hline 1 & 2.894666 & 2.889404 & -0.411162 & 1 & -2.638138 & -2.139683 & -2.132118 \\
\hline 6 & -1.371285 & 0.297113 & 0.106238 & 1 & -0.464770 & -1.329594 & -1.253241 \\
\hline 6 & -2.594058 & 0.449565 & 0.793061 & & & & \\
\hline 6 & -3.750662 & -0.194793 & 0.360325 & \multicolumn{4}{|c|}{$\mathrm{E}(\mathrm{MeCN})=-1019.06872$} \\
\hline 6 & -3.725721 & -1.002066 & -0.780164 & \multicolumn{4}{|c|}{ NImag $=0$} \\
\hline 6 & -2.523937 & -1.164778 & -1.471632 & & & & \\
\hline 6 & -1.359067 & -0.535951 & -1.033832 & \multirow{2}{*}{\multicolumn{4}{|c|}{ 11. I-2c conformer $2(-1018.84558)$}} \\
\hline 1 & -2.630120 & 1.083368 & 1.676802 & & & & \\
\hline 1 & -4.678371 & -0.056881 & 0.910552 & 6 & 0.325144 & 2.558329 & 0.061214 \\
\hline 1 & -4.630086 & -1.496620 & -1.123852 & 1 & 1.325412 & 2.349312 & 0.447107 \\
\hline 1 & -2.487612 & -1.795617 & -2.356529 & 1 & 0.353389 & 2.644773 & -1.027239 \\
\hline \multirow[t]{2}{*}{1} & -0.431170 & -0.687641 & -1.575535 & 16 & -0.911257 & 1.226674 & 0.462217 \\
\hline & & & & 6 & -0.569389 & 1.184872 & 2.257795 \\
\hline \multicolumn{4}{|c|}{$\mathrm{E}(\mathrm{MeCN})=-1019.043375$} & 1 & 0.475606 & 0.946694 & 2.463056 \\
\hline \multicolumn{4}{|c|}{ NImag = $1(-251.4)$} & 1 & -1.237924 & 0.440842 & 2.689719 \\
\hline \multirow{3}{*}{\multicolumn{4}{|c|}{ 10. I-2 $c$ conformer $1(-1018.86150)$}} & 6 & -0.023311 & -0.256090 & -0.383507 \\
\hline & & & & 1 & -0.372354 & -0.016025 & -1.392795 \\
\hline & & & & 6 & -0.869762 & -1.487542 & 0.034828 \\
\hline 6 & -0.325206 & 2.867271 & -0.729792 & 6 & -0.350554 & -2.321041 & 1.213404 \\
\hline 16 & 0.891342 & 1.455876 & -0.614442 & 1 & -0.347072 & -1.761325 & 2.157143 \\
\hline 6 & -0.046609 & 0.521402 & 0.750107 & 1 & -1.019862 & -3.176859 & 1.354996 \\
\hline 6 & -1.349622 & 0.026728 & 0.164193 & 1 & 0.656832 & -2.712498 & 1.040289 \\
\hline 6 & 2.171055 & 2.421792 & 0.262587 & 1 & -0.815489 & -2.134366 & -0.860337 \\
\hline 6 & 0.835889 & -0.548194 & 1.470899 & 6 & -2.290726 & -1.019358 & 0.266041 \\
\hline 6 & 1.356869 & -1.656469 & 0.601036 & 1 & -2.844984 & -1.517639 & 1.059125 \\
\hline 6 & 2.294817 & -1.351020 & -0.346171 & 6 & -3.004721 & -0.368100 & -0.758084 \\
\hline 6 & 2.963561 & -2.426094 & -1.184742 & 6 & -4.506609 & -0.148750 & -0.575317 \\
\hline 6 & 0.043010 & -1.082555 & 2.676673 & 1 & -4.894647 & -0.554927 & 0.365151 \\
\hline 8 & 2.658409 & -0.122755 & -0.579801 & 1 & -4.729643 & 0.924491 & -0.620694 \\
\hline 1 & -1.261107 & 2.475304 & -1.130482 & 1 & -5.043675 & -0.617653 & -1.408904 \\
\hline 1 & 0.091446 & 3.596175 & -1.430633 & 8 & -2.465280 & 0.125109 & -1.792042 \\
\hline 1 & -0.504786 & 3.343202 & 0.239639 & 1 & -0.804881 & 2.176429 & 2.655375 \\
\hline 1 & 2.448285 & 3.256771 & -0.385995 & 1 & -0.063235 & 3.489381 & 0.482646 \\
\hline 1 & 1.770032 & 2.801236 & 1.206756 & 6 & 1.479702 & -0.249851 & -0.332005 \\
\hline 1 & 3.002132 & 1.735495 & 0.407078 & 6 & 2.176923 & 0.001608 & -1.526891 \\
\hline 1 & -0.265554 & 1.322503 & 1.468735 & 6 & 3.570824 & 0.002154 & -1.568307 \\
\hline 1 & -0.319071 & -0.279034 & 3.331154 & 6 & 4.303723 & -0.234716 & -0.404795 \\
\hline 1 & -0.821689 & -1.672423 & 2.354181 & 6 & 3.630865 & -0.480081 & 0.793465 \\
\hline
\end{tabular}




$\begin{array}{rrrr}6 & 2.236061 & -0.493050 & 0.829259 \\ 1 & 1.612862 & 0.188176 & -2.437368 \\ 1 & 4.082376 & 0.189467 & -2.508515 \\ 1 & 5.389964 & -0.231854 & -0.431054 \\ 1 & 4.192475 & -0.671709 & 1.703925 \\ 1 & 1.738186 & -0.712666 & 1.766627\end{array}$

$\mathrm{E}(\mathrm{MeCN})=-1019.0614204$

NImag $=0$

12. I-2 $c$ conformer3 (-1018.8615123)

$\begin{array}{lrrr}6 & 2.187883 & 2.411666 & 0.246765 \\ 1 & 1.811094 & 2.754062 & 1.214832 \\ 1 & 3.031507 & 1.733180 & 0.347282 \\ 16 & 0.894037 & 1.455183 & -0.620104 \\ 6 & -0.313414 & 2.876108 & -0.723049 \\ 1 & -0.473711 & 3.357787 & 0.246937 \\ 1 & -1.258775 & 2.489651 & -1.106585 \\ 6 & -0.045239 & 0.526740 & 0.748032 \\ 1 & -0.264395 & 1.331335 & 1.462637 \\ 6 & 0.835146 & -0.539699 & 1.475848 \\ 6 & 0.041915 & -1.062990 & 2.686036 \\ 1 & -0.827632 & -1.648325 & 2.368269 \\ 1 & 0.687821 & -1.717716 & 3.280378 \\ 1 & -0.312594 & -0.253861 & 3.337793 \\ 1 & 1.691548 & 0.035185 & 1.865845 \\ 6 & 1.352372 & -1.656511 & 0.614760 \\ 1 & 1.036828 & -2.674825 & 0.820004 \\ 6 & 2.281858 & -1.361337 & -0.343500 \\ 6 & 2.944641 & -2.445194 & -1.175345 \\ 1 & 2.598114 & -3.450052 & -0.910215 \\ 1 & 2.755471 & -2.273410 & -2.242819 \\ 1 & 4.032748 & -2.402927 & -1.039018 \\ 8 & 2.642977 & -0.135548 & -0.594507 \\ 1 & 0.097326 & 3.598705 & -1.433821 \\ 1 & 2.432544 & 3.271810 & -0.381635 \\ 6 & -1.347537 & 0.030485 & 0.162826 \\ 6 & -2.559215 & 0.517187 & 0.679233 \\ 6 & -3.783364 & 0.051940 & 0.199234 \\ 6 & -3.815195 & -0.906188 & -0.815670 \\ 6 & -2.617347 & -1.394858 & -1.339849 \\ 6 & -1.391226 & -0.932457 & -0.859577 \\ 1 & -2.542361 & 1.260639 & 1.473387 \\ 1 & -4.709097 & 0.438483 & 0.617149 \\ 1 & -4.766891 & -1.267442 & -1.196217 \\ 1 & -2.633938 & -2.141514 & -2.129322 \\ \mathrm{E}(\mathrm{MeCN}) \\ \mathrm{NImag}=0 & -1019.0686262 & & \\ & & & \\ & & & \\ 1 & & & \\ 1 & & & \\ 1 & & & \\ 1 & & & \\ 1 & & & \end{array}$

13. I-2 $c$ conformer4 (-1018.85740)

$\begin{array}{cccc}6 & 1.874255 & 2.123293 & 0.228181 \\ 1 & 1.456196 & 3.09883 & 0.484934 \\ 1 & 2.049941 & 1.507537 & 1.119695 \\ 16 & 0.717796 & 1.226774 & -0.873616 \\ 6 & -0.671428 & 2.469086 & -0.778030 \\ 1 & -1.015638 & 2.611136 & 0.249238 \\ 1 & -1.497796 & 2.119807 & -1.396678 \\ 6 & -0.040281 & -0.215000 & 0.143701 \\ 1 & 0.404027 & -0.037892 & 1.128926 \\ 6 & 0.748684 & -1.429231 & -0.411154 \\ 6 & 0.235302 & -2.023619 & -1.726330 \\ 1 & 0.259605 & -1.291751 & -2.544771 \\ 1 & 0.887604 & -2.853608 & -2.019780 \\ 1 & -0.783147 & -2.414841 & -1.640068 \\ 1 & 0.633107 & -2.189934 & 0.378925 \\ 6 & 2.193573 & -0.999326 & -0.519780 \\ 1 & 2.723986 & -1.275317 & -1.428049 \\ 6 & 2.936500 & -0.690361 & 0.631824 \\ 6 & 4.454700 & -0.548622 & 0.508378 \\ 1 & 4.820378 & -0.664789 & -0.517605 \\ 1 & 4.774873 & 0.426324 & 0.896750 \\ 1 & 4.935921 & -1.307165 & 1.138129 \\ 8 & 2.421439 & -0.461822 & 1.769926 \\ 6 & -1.543029 & -0.230859 & 0.243346 \\ 1 & -0.278892 & 3.404604 & -1.184376 \\ 1 & 2.808125 & 2.231000 & -0.325194 \\ 6 & -2.125420 & -0.138602 & 1.517828 \\ 6 & -3.510003 & -0.192707 & 1.684466 \\ 6 & -4.342236 & -0.324829 & 0.572760 \\ 6 & -3.780427 & -0.406590 & -0.703625 \\ 6 & -2.396347 & -0.364028 & -0.866301 \\ 1 & -1.481399 & -0.038770 & 2.388107 \\ 1 & -3.936084 & -0.128913 & 2.682060 \\ 1 & -5.420946 & -0.363223 & 0.697749 \\ 1 & -4.421523 & -0.507826 & -1.575300 \\ 1 & -1.977641 & -0.430713 & -1.865834 \\ \mathrm{E}(\mathrm{MeCN}) \\ \mathrm{NImag}=0 & -1019.0661547 & & \\ & & & \\ 1 & & & \\ 1 & & & \\ 1 & & & \\ 1 & & & \\ 1 & & & \end{array}$

14. TS-2t (-1018.83403)

$\begin{array}{crrr}6 & 2.394698 & -2.384435 & -0.877492 \\ 1 & 2.656851 & -1.414347 & -1.305663 \\ 1 & 3.072940 & -2.633482 & -0.059080 \\ 16 & 0.688887 & -2.297260 & -0.183023 \\ 6 & -0.230943 & -2.018654 & -1.736347 \\ 1 & 0.341835 & -1.352902 & -2.385354 \\ 1 & -1.201373 & -1.552982 & -1.492185 \\ 6 & 0.524337 & -0.848485 & 0.837546 \\ 1 & 0.929985 & -1.181015 & 1.794688\end{array}$




\begin{tabular}{|c|c|c|c|c|c|c|c|}
\hline 6 & -1.503662 & -0.651053 & 1.335043 & 1 & 4.023991 & -0.747346 & -1.571814 \\
\hline 6 & -1.382086 & -0.863359 & 2.832294 & 1 & 4.622851 & -1.998776 & -0.487681 \\
\hline 1 & -0.863131 & -0.025602 & 3.312933 & 8 & 2.834518 & -0.553755 & 0.862569 \\
\hline 1 & -2.384783 & -0.923331 & 3.274761 & 1 & -4.371770 & 1.473607 & -0.490279 \\
\hline 1 & -0.852484 & -1.788385 & 3.086866 & 1 & -4.539605 & -1.241154 & -0.343158 \\
\hline 1 & -1.842698 & -1.531215 & 0.789773 & 6 & -0.049087 & 0.894855 & -0.056185 \\
\hline 6 & -2.056821 & 0.553879 & 0.866950 & 6 & 0.665462 & 1.431141 & -1.138526 \\
\hline 1 & -2.026693 & 1.433227 & 1.505979 & 6 & 1.444239 & 2.578800 & -0.985053 \\
\hline 6 & -2.641938 & 0.661620 & -0.421108 & 6 & 1.499189 & 3.230406 & 0.246077 \\
\hline 6 & -3.366021 & 1.957884 & -0.778717 & 6 & 0.780792 & 2.716499 & 1.329045 \\
\hline 1 & -3.381776 & 2.683348 & 0.040818 & 6 & 0.024956 & 1.557173 & 1.183091 \\
\hline 1 & -2.888423 & 2.419448 & -1.651856 & 1 & 0.625546 & 0.929042 & -2.101787 \\
\hline 1 & -4.397312 & 1.725137 & -1.068811 & 1 & 2.005805 & 2.964879 & -1.831980 \\
\hline 8 & -2.609353 & -0.241147 & -1.299008 & 1 & 2.103124 & 4.125643 & 0.366145 \\
\hline 1 & -0.356354 & -2.996936 & -2.208048 & 1 & 0.830408 & 3.207353 & 2.297466 \\
\hline 1 & 2.423184 & -3.169108 & -1.638636 & 1 & -0.492364 & 1.143386 & 2.045929 \\
\hline 6 & 1.130483 & 0.416833 & 0.371479 & & & & \\
\hline 6 & 2.153246 & 1.020891 & 1.129541 & \multicolumn{4}{|c|}{$\mathrm{E}(\mathrm{MeCN})=-1019.0270613$} \\
\hline 6 & 2.704642 & 2.244225 & 0.756720 & \multicolumn{4}{|c|}{ NImag = $1(-362.2)$} \\
\hline 6 & 2.252321 & 2.894722 & -0.394643 & & & & \\
\hline 6 & 1.232040 & 2.317803 & -1.150905 & \multicolumn{4}{|c|}{ 16. I-2 $t$ conformer1 $(-1018.8455827)$} \\
\hline 6 & 0.661206 & 1.101976 & -0.768144 & & & & \\
\hline 1 & 2.518416 & 0.517589 & 2.022688 & 6 & 2.317791 & -2.265681 & -0.729393 \\
\hline 1 & 3.494607 & 2.685605 & 1.359239 & 1 & 2.677100 & -1.251788 & -0.918017 \\
\hline 1 & 2.685634 & 3.845851 & -0.692320 & 1 & 2.789037 & -2.673656 & 0.167705 \\
\hline 1 & 0.859141 & 2.825445 & -2.036909 & 16 & 0.515675 & -2.243915 & -0.466088 \\
\hline 1 & -0.163954 & 0.695905 & -1.341763 & 6 & -0.117076 & -1.577362 & -2.033274 \\
\hline & & & & 1 & 0.630749 & -0.913682 & -2.471815 \\
\hline \multicolumn{4}{|c|}{$\mathrm{E}(\mathrm{MeCN})=-1019.0413165$} & 1 & -1.050514 & -0.997921 & -1.793317 \\
\hline \multirow{2}{*}{\multicolumn{4}{|c|}{ NImag = $1(-261.2)$}} & 6 & 5818 & 1720 & 0.813162 \\
\hline & & & & 1 & 0.735476 & -1.314355 & 1.673298 \\
\hline \multirow{2}{*}{\multicolumn{4}{|c|}{ 15. TS- $2 t$ conformer2 (-1018.8143605) }} & 6 & -1.346967 & -0.844593 & 1.087495 \\
\hline & & & & 6 & -1.564262 & -1.434905 & 2.492355 \\
\hline 6 & -3.471963 & -1.415760 & -0.493335 & 1 & -1.157846 & -0.766253 & 3.262202 \\
\hline 1 & -3.245387 & -1.494094 & -1.560179 & 1 & -2.635546 & -1.546760 & 2.683299 \\
\hline 1 & -3.167395 & -2.325684 & 0.020590 & 1 & -1.091156 & -2.419218 & 2.615576 \\
\hline 16 & -2.558481 & -0.017476 & 0.234915 & 1 & -1.788423 & -1.541250 & 0.357152 \\
\hline 6 & -3.329606 & 1.337561 & -0.794311 & 6 & -2.045746 & 0.461976 & 0.912952 \\
\hline 1 & -3.260598 & 1.085122 & -1.855381 & 1 & -2.241679 & 1.059398 & 1.799283 \\
\hline 1 & -2.753265 & 2.239856 & -0.583847 & 6 & -2.534493 & 0.836324 & -0.319948 \\
\hline 6 & -0.817834 & -0.380361 & -0.218315 & 6 & -3.371520 & 2.105123 & -0.438473 \\
\hline 1 & -0.841217 & -0.714409 & -1.260192 & 1 & -3.563994 & 2.584400 & 0.527813 \\
\hline 6 & 0.277823 & -1.846683 & 0.623694 & 1 & -2.868982 & 2.827370 & -1.095779 \\
\hline 6 & -0.690172 & -2.984705 & 0.868705 & 1 & -4.331407 & 1.865753 & -0.912802 \\
\hline 1 & -1.046407 & -3.429732 & -0.069158 & 8 & -2.310090 & 0.204437 & -1.424207 \\
\hline 1 & -0.177177 & -3.780675 & 1.423717 & 1 & -0.314822 & -2.431198 & -2.685453 \\
\hline 1 & -1.556078 & -2.676583 & 1.469166 & 1 & 2.523993 & -2.918466 & -1.580957 \\
\hline 1 & 0.547330 & -1.288518 & 1.519687 & 6 & 0.984266 & 0.404054 & 0.460559 \\
\hline 6 & 1.355146 & -2.033762 & -0.255063 & 6 & 2.139450 & 0.716312 & 1.195583 \\
\hline 1 & 1.252638 & -2.725450 & -1.090098 & 6 & 2.885898 & 1.862571 & 0.914477 \\
\hline 6 & 2.608165 & -1.352873 & -0.059536 & 6 & 2.486270 & 2.709933 & -0.118217 \\
\hline 6 & 3.739889 & -1.666716 & -1.045406 & 6 & 1.338259 & 2.408907 & -0.856129 \\
\hline 1 & 3.477148 & -2.430263 & -1.785629 & 6 & 0.580993 & 1.272116 & -0.571644 \\
\hline
\end{tabular}




\begin{tabular}{|c|c|c|c|c|c|c|c|}
\hline 1 & 2.449460 & 0.064060 & 2.010168 & \multicolumn{4}{|c|}{ 18. TS-2R (Optimized at the HF/6-31G* level) } \\
\hline 1 & 3.772270 & 2.088841 & 1.501004 & & & & \\
\hline 1 & 3.062735 & 3.603108 & -0.344917 & 6 & -0.888178 & -3.345664 & -0.811861 \\
\hline 1 & 1.017579 & 3.073026 & -1.654304 & 1 & -0.399689 & -3.060686 & -1.733353 \\
\hline \multirow[t]{2}{*}{1} & -0.316185 & 1.057400 & -1.146124 & 1 & -0.196395 & -3.868999 & -0.173687 \\
\hline & & & & 16 & -1.479454 & -1.878010 & 0.060474 \\
\hline \multicolumn{4}{|c|}{$\mathrm{E}(\mathrm{MeCN})=-1019.06091$} & 6 & -2.698947 & -1.286781 & -1.133801 \\
\hline \multirow{2}{*}{\multicolumn{4}{|c|}{ NImag $=0$}} & 1 & -2.225202 & -1.075765 & -2.082264 \\
\hline & & & & 1 & -3.145933 & -0.388453 & -0.739391 \\
\hline \multirow{2}{*}{\multicolumn{4}{|c|}{ 17. I-2 $t$ conformer2 (-1018.8433294) }} & 6 & -0.022739 & -0.596339 & -0.100892 \\
\hline & & & & 1 & 0.286720 & -0.773508 & -1.118038 \\
\hline 6 & -2.751550 & -2.233747 & -0.282696 & 6 & 1.200438 & -0.850724 & 0.867803 \\
\hline 16 & -1.010279 & -1.936404 & -0.738430 & 6 & 1.212886 & -2.192721 & 1.606270 \\
\hline 6 & -0.340255 & -0.854386 & 0.699047 & 1 & 1.452135 & -3.019752 & 0.944105 \\
\hline 6 & 1.113052 & -0.606734 & 0.360323 & 1 & 2.003244 & -2.152695 & 2.345176 \\
\hline 6 & -1.191169 & -0.783817 & -2.146169 & 1 & 0.287740 & -2.421903 & 2.134410 \\
\hline 6 & -1.311357 & 0.354384 & 1.089366 & 1 & 1.112494 & -0.064990 & 1.607702 \\
\hline 6 & -0.713781 & 1.717132 & 1.035171 & 6 & 2.460593 & -0.647845 & 0.084086 \\
\hline 6 & -0.790624 & 2.450218 & -0.131966 & 1 & 3.024282 & -1.520226 & -0.201451 \\
\hline 6 & -0.279208 & 3.886333 & -0.148711 & 6 & 2.904988 & 0.630651 & -0.189507 \\
\hline 6 & -1.903685 & 0.037570 & 2.475998 & 6 & 4.241171 & 0.781056 & -0.910752 \\
\hline 8 & -1.248550 & 1.995783 & -1.248912 & 1 & 4.712011 & -0.165206 & -1.159214 \\
\hline 1 & -2.762260 & -2.771995 & 0.667245 & 1 & 4.094747 & 1.353741 & -1.822695 \\
\hline 1 & -3.191774 & -2.858410 & -1.063240 & 1 & 4.916725 & 1.354694 & -0.282696 \\
\hline 1 & -3.291700 & -1.290633 & -0.192089 & 8 & 2.300850 & 1.678765 & 0.099965 \\
\hline 1 & -0.278074 & -0.878780 & -2.735951 & 6 & -0.701650 & 0.746617 & 0.036637 \\
\hline 1 & -2.043020 & -1.135875 & -2.734314 & 1 & -3.452576 & -2.053842 & -1.248546 \\
\hline 1 & -1.320345 & 0.266235 & -1.777599 & 1 & -1.739204 & -3.980125 & -1.017572 \\
\hline 1 & -0.368093 & -1.612768 & 1.492217 & 6 & -1.317714 & 1.118929 & 1.227068 \\
\hline 1 & -2.381496 & -0.952151 & 2.523718 & 6 & -1.959528 & 2.340200 & 1.333901 \\
\hline 1 & -1.125025 & 0.065916 & 3.248936 & 6 & -1.980072 & 3.209398 & 0.254626 \\
\hline 1 & -2.653507 & 0.790317 & 2.736084 & 6 & -1.352815 & 2.852505 & -0.926900 \\
\hline 1 & -2.124995 & 0.355141 & 0.352057 & 6 & -0.720646 & 1.625765 & -1.036116 \\
\hline 1 & -0.271286 & 2.130557 & 1.937246 & 1 & -1.286514 & 0.462381 & 2.079475 \\
\hline 1 & 0.562838 & 3.978354 & -0.847890 & 1 & -2.429527 & 2.617389 & 2.260321 \\
\hline 1 & -1.068850 & 4.550985 & -0.520424 & 1 & -2.469133 & 4.163123 & 0.339797 \\
\hline 1 & 0.044409 & 4.237145 & 0.837592 & 1 & -1.342901 & 3.532585 & -1.759270 \\
\hline 6 & 2.092384 & -1.348802 & 1.036364 & 1 & -0.205287 & 1.369998 & -1.943501 \\
\hline 6 & 3.448354 & -1.174509 & 0.755169 & & & & \\
\hline 6 & 3.839843 & -0.257627 & -0.219784 & \multicolumn{4}{|c|}{$\mathrm{E}(\mathrm{MeCN})=-1019.0434372$} \\
\hline 6 & 2.871818 & 0.483982 & -0.901594 & \multicolumn{4}{|c|}{ NImag $=1(-63.0)$} \\
\hline 6 & 1.516008 & 0.323128 & -0.614738 & & & & \\
\hline 1 & 1.792834 & -2.064014 & 1.799858 & \multicolumn{4}{|c|}{ 19. TS-2E (-1018.84314) } \\
\hline 1 & 4.191662 & -1.755189 & 1.294687 & & & & \\
\hline 1 & 4.893780 & -0.118852 & -0.446422 & 16 & 0.911460 & -2.217812 & -0.484253 \\
\hline 1 & 3.172760 & 1.205113 & -1.656750 & 6 & 0.411400 & -1.442333 & -2.062910 \\
\hline \multirow{2}{*}{1} & 0.776041 & 0.916355 & -1.143476 & 6 & 2.715672 & -1.974425 & -0.510419 \\
\hline & & & & 6 & 0.120534 & -0.748326 & 0.870200 \\
\hline \multicolumn{4}{|c|}{$\mathrm{E}(\mathrm{MeCN})=-1019.0586405$} & 6 & -1.394991 & -1.079275 & 0.814837 \\
\hline \multirow{4}{*}{\multicolumn{4}{|c|}{ NImag $=0$}} & 6 & -1.716873 & -2.090422 & 1.929014 \\
\hline & & & & 6 & -2.226149 & 0.157896 & 0.874816 \\
\hline & & & & 6 & -2.645489 & 0.709580 & -0.327680 \\
\hline & & & & 8 & -2.258270 & 0.279464 & -1.473891 \\
\hline
\end{tabular}




\begin{tabular}{|c|c|c|c|c|c|c|c|}
\hline 6 & -3.595775 & 1.900534 & -0.319034 & 1 & 0.910206 & -3.938586 & 0.251034 \\
\hline 1 & -0.599775 & -1.020106 & -1.936674 & 1 & 0.943197 & -1.552001 & 1.172379 \\
\hline 1 & 0.426749 & -2.212327 & -2.837428 & 1 & 2.665980 & -1.799852 & -1.378671 \\
\hline 1 & 1.108317 & -0.637197 & -2.303307 & 1 & 4.564906 & 0.215870 & -1.240145 \\
\hline 1 & 3.121957 & -2.409945 & -1.426921 & 1 & 5.322540 & 0.140135 & 0.379366 \\
\hline 1 & 2.948410 & -0.907561 & -0.455494 & 1 & 5.098022 & -1.338999 & -0.600489 \\
\hline 1 & 3.134974 & -2.493605 & 0.354489 & 6 & 0.482203 & 0.786787 & -0.405299 \\
\hline 1 & 0.562281 & -1.152570 & 1.788142 & 6 & 0.448082 & 1.248766 & 0.921106 \\
\hline 1 & -1.075144 & -2.981379 & 1.885663 & 6 & 0.035208 & 2.550569 & 1.209605 \\
\hline 1 & -1.593092 & -1.634766 & 2.919942 & 6 & -0.357156 & 3.416821 & 0.187754 \\
\hline 1 & -2.756803 & -2.417771 & 1.841168 & 6 & -0.330682 & 2.970472 & -1.134279 \\
\hline 1 & -1.622187 & -1.546735 & -0.153352 & 6 & 0.085056 & 1.670972 & -1.423380 \\
\hline 1 & -2.538587 & 0.550742 & 1.837641 & 1 & 0.770817 & 0.601291 & 1.727067 \\
\hline 1 & -3.105868 & 2.773401 & -0.770758 & 1 & 0.022927 & 2.887785 & 2.242866 \\
\hline 1 & -4.471680 & 1.674689 & -0.939116 & 1 & -0.679604 & 4.428451 & 0.419200 \\
\hline 1 & -3.932815 & 2.170865 & 0.687757 & 1 & -0.633428 & 3.631901 & -1.942046 \\
\hline 6 & 0.785837 & 0.546404 & 0.552255 & 1 & 0.103400 & 1.331673 & -2.457145 \\
\hline 6 & 0.443891 & 1.381981 & -0.532509 & & & & \\
\hline 6 & 1.177041 & 2.539080 & -0.779591 & \multicolumn{4}{|c|}{$\mathrm{E}(\mathrm{MeCN})=-1019.1200179$} \\
\hline 6 & 2.263111 & 2.893083 & 0.029647 & \multicolumn{4}{|c|}{ NImag $=0$} \\
\hline 6 & 2.611435 & 2.079050 & 1.105706 & & & & \\
\hline 6 & 1.879678 & 0.917780 & 1.360427 & \multirow{2}{*}{\multicolumn{4}{|c|}{ 21. TS-2'c (-1018.83422) }} \\
\hline 1 & -0.410722 & 1.130250 & -1.157971 & & & & \\
\hline 1 & 0.892437 & 3.177516 & -1.611889 & 6 & 0.548240 & 2.799125 & -1.105052 \\
\hline 1 & 2.827660 & 3.798577 & -0.176718 & 1 & 1.530820 & 2.693320 & -0.639758 \\
\hline 1 & 3.444514 & 2.345231 & 1.750815 & 1 & 0.628050 & 2.662170 & -2.185519 \\
\hline 1 & 2.146246 & 0.298313 & 2.214630 & 16 & -0.598614 & 1.498911 & -0.471197 \\
\hline \multirow{2}{*}{\multicolumn{4}{|c|}{$\mathrm{E}(\mathrm{MeCN})=-1019.0550204$}} & 6 & -0.584955 & 1.971833 & 1.286851 \\
\hline & & & & 1 & 0.433152 & 1.980837 & 1.679502 \\
\hline \multirow{2}{*}{\multicolumn{4}{|c|}{ NImag = $1(-169.1)$}} & 1 & -1.246416 & 1.252653 & 1.774901 \\
\hline & & & & 6 & 0.208616 & -0.075776 & -0.634091 \\
\hline \multirow{2}{*}{\multicolumn{4}{|c|}{ 20. PC-2 (-1018.92767) }} & 1 & 0.038920 & -0.336897 & -1.680208 \\
\hline & & & & 6 & -1.151541 & -1.556515 & 0.143226 \\
\hline 16 & -3.625129 & -1.408289 & 0.119727 & 6 & -0.357152 & -2.783374 & -0.242923 \\
\hline 6 & -3.325005 & -0.366882 & 1.590585 & 1 & -0.753385 & -3.652250 & 0.299844 \\
\hline 6 & -3.727561 & -0.104502 & -1.156020 & 1 & -0.451343 & -2.999757 & -1.313658 \\
\hline 6 & 0.879850 & -0.597602 & -0.799374 & 1 & 0.704967 & -2.695462 & 0.001116 \\
\hline 6 & 0.999337 & -1.779223 & 0.109562 & 6 & -2.446374 & -1.389037 & -0.361003 \\
\hline 6 & 0.423754 & -3.120214 & -0.293023 & 1 & -2.754454 & -1.954433 & -1.237987 \\
\hline 6 & 2.269355 & -1.256451 & -0.523087 & 6 & -3.358681 & -0.468126 & 0.226367 \\
\hline 6 & 3.294858 & -0.573499 & 0.318756 & 6 & -4.796321 & -0.440563 & -0.288309 \\
\hline 8 & 3.062716 & -0.190374 & 1.455544 & 1 & -4.979702 & -1.156310 & -1.096168 \\
\hline 6 & 4.658969 & -0.373307 & -0.319060 & 1 & -5.480322 & -0.659329 & 0.540423 \\
\hline 1 & -3.229098 & -1.039880 & 2.446956 & 1 & -5.040869 & 0.568411 & -0.642320 \\
\hline 1 & -4.164566 & 0.312271 & 1.769914 & 8 & -3.053582 & 0.329304 & 1.146763 \\
\hline 1 & -2.400413 & 0.208442 & 1.483719 & 1 & -1.031030 & 2.968162 & 1.342728 \\
\hline 1 & -4.567614 & 0.570245 & -0.962690 & 1 & 0.119526 & 3.781949 & -0.889219 \\
\hline 1 & -2.798138 & 0.470714 & -1.208656 & 6 & 1.625213 & -0.192785 & -0.222715 \\
\hline 1 & -3.892715 & -0.604260 & -2.114446 & 6 & 2.628181 & -0.448246 & -1.182885 \\
\hline 1 & 0.537437 & -0.847000 & -1.802868 & 6 & 3.958775 & -0.633474 & -0.814302 \\
\hline 1 & -0.651341 & -3.155405 & -0.082588 & 6 & 4.335623 & -0.559288 & 0.529080 \\
\hline 1 & 0.560221 & -3.303416 & -1.365813 & 6 & 3.358890 & -0.321702 & 1.497889 \\
\hline
\end{tabular}




$\begin{array}{lrrr}6 & 2.023650 & -0.153149 & 1.131838 \\ 1 & 2.350472 & -0.499647 & -2.233453 \\ 1 & 4.705928 & -0.828291 & -1.579743 \\ 1 & 5.373845 & -0.697784 & 0.817588 \\ 1 & 3.633284 & -0.287076 & 2.549327 \\ 1 & 1.273961 & -0.033893 & 1.907789 \\ 1 & -1.001914 & -1.216922 & 1.166042 \\ & & \\ \mathrm{E}(\mathrm{MeCN})=-1019.0412037 & \\ \text { NImag = } 1(-246.6)\end{array}$

22. TS-2'c conformer2 (-1018.8319452)

\begin{tabular}{|c|c|c|c|}
\hline 6 & 2.010195 & 2.019903 & 0.657070 \\
\hline 1 & 1.764381 & 2.463477 & 1.625546 \\
\hline 1 & 2.520739 & 1.063333 & 0.793772 \\
\hline 16 & 0.484471 & 1.657631 & -0.260402 \\
\hline 6 & -0.401642 & 3.257869 & 0.038820 \\
\hline 1 & -0.462378 & 3.465096 & 1.110741 \\
\hline 1 & -1.403679 & 3.144755 & -0.380249 \\
\hline 6 & -0.303102 & 0.435284 & 0.751613 \\
\hline 1 & -0.370655 & 0.802601 & 1.779377 \\
\hline 6 & 0.865925 & -1.374189 & 0.839402 \\
\hline 6 & 0.310162 & -1.925887 & 2.132243 \\
\hline 1 & 0.488055 & -3.009066 & 2.172906 \\
\hline 1 & 0.802623 & -1.481822 & 3.005539 \\
\hline 1 & -0.769528 & -1.770197 & 2.221892 \\
\hline 6 & 2.255869 & -1.270011 & 0.671894 \\
\hline 1 & 2.922528 & -1.400701 & 1.522590 \\
\hline 6 & 2.786415 & -0.988811 & -0.621158 \\
\hline 6 & 4.290196 & -1.129856 & -0.851589 \\
\hline 1 & 4.847162 & -1.353491 & 0.064524 \\
\hline 1 & 4.467595 & -1.938832 & -1.570731 \\
\hline 1 & 4.687418 & -0.213312 & -1.303696 \\
\hline 8 & 2.068809 & -0.610710 & -1.577456 \\
\hline 1 & 0.131408 & 4.060093 & -0.481012 \\
\hline 1 & 2.616604 & 2.703833 & 0.060098 \\
\hline 6 & -1.593153 & 0.003663 & 0.172070 \\
\hline 6 & -2.761614 & -0.027470 & 0.958721 \\
\hline 6 & -3.964463 & -0.507475 & 0.442072 \\
\hline 6 & -4.036513 & -0.955143 & -0.878641 \\
\hline 6 & -2.885545 & -0.933430 & -1.671150 \\
\hline 6 & -1.675920 & -0.472475 & -1.155873 \\
\hline 1 & -2.721074 & 0.332490 & 1.984855 \\
\hline 1 & -4.850756 & -0.522015 & 1.071832 \\
\hline 1 & -2.925497 & -1.291868 & -2.696772 \\
\hline 1 & -0.777088 & -0.493599 & -1.767975 \\
\hline 1 & 0.325182 & -1.667855 & -0.056283 \\
\hline 1 & -4.975896 & -1.321157 & -1.283842 \\
\hline
\end{tabular}

$\mathrm{E}(\mathrm{MeCN})=-1019.03851)$

NImag $=1(-253.3)$
23. I-2'c conformer1 (-1018.86038)

$$
\begin{array}{rcc}
0.822557 & 2.667922 & -0.684189 \\
1.625221 & 2.386184 & 0.000026 \\
1.181785 & 2.641547 & -1.716177 \\
-0.657867 & 1.526035 & -0.556366 \\
-0.935403 & 1.655161 & 1.237633 \\
0.026104 & 1.661892 & 1.755580 \\
-1.578295 & 0.814582 & 1.564445 \\
0.216679 & -0.146189 & -0.842330 \\
0.301902 & -0.113460 & -1.937190 \\
-0.866003 & -1.175031 & -0.438840 \\
-0.592954 & -2.511019 & -1.155831 \\
-1.300534 & -3.265777 & -0.800428 \\
-0.731283 & -2.409214 & -2.240378 \\
0.424358 & -2.883284 & -0.976773 \\
-2.238586 & -0.608062 & -0.681142 \\
-2.604432 & -0.583462 & -1.706814 \\
-3.152830 & -0.507930 & 0.380745 \\
-4.613169 & -0.190000 & 0.059699 \\
-4.803153 & -0.056106 & -1.010862 \\
-5.247071 & -1.005328 & 0.429572 \\
-4.923090 & 0.715793 & 0.595287 \\
-2.834072 & -0.606687 & 1.605458 \\
-1.453801 & 2.604531 & 1.396387 \\
0.457055 & 3.672547 & -0.456626 \\
1.590712 & -0.276962 & -0.239909 \\
2.728109 & -0.155095 & -1.053977 \\
4.010935 & -0.295912 & -0.524901 \\
4.178344 & -0.556488 & 0.835959 \\
3.056701 & -0.683538 & 1.658253 \\
1.774022 & -0.549864 & 1.126779 \\
2.604027 & 0.041311 & -2.116855 \\
4.876763 & -0.205907 & -1.175255 \\
5.175767 & -0.667602 & 1.252279 \\
3.179021 & -0.897459 & 2.716469 \\
0.912517 & -0.675936 & 1.775179 \\
-0.799105 & -1.339885 & 0.642628
\end{array}
$$

$\mathrm{E}(\mathrm{MeCN})=-1019.0717832$

NImag $=0$

24. I-2'c conformer2 (-1018.8577103)

$\begin{array}{cccc}6 & 1.766695 & 2.608820 & -0.017247 \\ 1 & 1.117321 & 3.169007 & 0.661205 \\ 1 & 2.639108 & 2.223770 & 0.507530 \\ 16 & 0.881840 & 1.160215 & -0.709612 \\ 6 & -0.454879 & 2.193901 & -1.528227 \\ 1 & -0.964758 & 2.861976 & -0.828301 \\ 1 & -1.171795 & 1.502980 & -1.972344 \\ 6 & -0.143999 & 0.483914 & 0.736995 \\ 1 & -0.270063 & 1.364276 & 1.379263\end{array}$




\begin{tabular}{|c|c|c|c|c|c|c|c|}
\hline 6 & 0.823412 & -0.552009 & 1.405075 & 1 & 4.159293 & 1.522250 & -1.623113 \\
\hline 6 & 0.859259 & -0.323256 & 2.921781 & 8 & 3.009460 & -0.827481 & -1.004843 \\
\hline 1 & 1.449375 & -1.106866 & 3.408143 & 1 & -1.795135 & -3.841853 & 0.608199 \\
\hline 1 & 1.321694 & 0.641881 & 3.170166 & 1 & -2.701713 & -2.967186 & -1.710164 \\
\hline 1 & -0.146799 & -0.336727 & 3.360550 & 6 & -0.883404 & 0.667586 & -0.190292 \\
\hline 6 & 2.180431 & -0.485859 & 0.746371 & 6 & -0.426458 & 1.858629 & -0.791603 \\
\hline 1 & 3.027183 & -0.147194 & 1.341762 & 6 & -1.160415 & 3.040614 & -0.700465 \\
\hline 6 & 2.404040 & -1.305407 & -0.383111 & 6 & -2.381767 & 3.066358 & -0.025521 \\
\hline 6 & 3.827061 & -1.443842 & -0.924097 & 6 & -2.850379 & 1.897418 & 0.578476 \\
\hline 1 & 4.570107 & -0.894734 & -0.335171 & 6 & -2.105270 & 0.721150 & 0.510324 \\
\hline 1 & 4.104483 & -2.504717 & -0.938268 & 1 & 0.516352 & 1.845103 & -1.329348 \\
\hline 1 & 3.863954 & -1.094607 & -1.963250 & 1 & -0.779055 & 3.943583 & -1.170471 \\
\hline 8 & 1.474863 & -1.869659 & -1.020168 & 1 & -2.959347 & 3.984616 & 0.034187 \\
\hline 1 & 0.030920 & 2.774201 & -2.317276 & 1 & -3.793063 & 1.903759 & 1.120123 \\
\hline 1 & 2.070358 & 3.248929 & -0.850854 & 1 & -2.475654 & -0.157834 & 1.030602 \\
\hline 6 & -1.497051 & -0.011695 & 0.297692 & 1 & 1.646899 & -1.553888 & 1.028186 \\
\hline 6 & -2.661611 & 0.622432 & 0.752494 & & & & \\
\hline 6 & -3.920882 & 0.149460 & 0.379247 & \multicolumn{4}{|c|}{$E(\mathrm{MeCN})=-1019.03595$} \\
\hline 6 & -4.024662 & -0.958387 & -0.462734 & \multicolumn{4}{|c|}{ NImag = $1(-357.4)$} \\
\hline 6 & -2.867179 & -1.594074 & -0.922197 & & & & \\
\hline 6 & -1.606851 & -1.132601 & -0.544297 & \multirow{2}{*}{\multicolumn{4}{|c|}{ 26. TS-2' $t$ conformer2 (-1018.8326419) }} \\
\hline 1 & -2.583718 & 1.485116 & 1.411474 & & & & \\
\hline 1 & -4.816023 & 0.646074 & 0.744296 & 6 & -1.654304 & 2.614400 & -0.110457 \\
\hline 1 & -5.003484 & -1.327427 & -0.757947 & 1 & -1.686287 & 2.900445 & -1.164581 \\
\hline 1 & -2.945993 & -2.459361 & -1.575029 & 1 & -2.313284 & 1.741744 & 0.043912 \\
\hline 1 & -0.693954 & -1.620948 & -0.885693 & 16 & 0.055439 & 2.127254 & 0.313587 \\
\hline \multirow[t]{2}{*}{1} & 0.391019 & -1.540174 & 1.208414 & 6 & 0.989120 & 3.131466 & -0.933775 \\
\hline & & & & 1 & 0.606369 & 2.917755 & -1.934372 \\
\hline \multicolumn{4}{|c|}{$\mathrm{E}(\mathrm{MeCN})=-1019.0658946$} & 1 & 2.037577 & 2.837333 & -0.859413 \\
\hline \multirow{2}{*}{\multicolumn{4}{|c|}{ NImag $=0$}} & 6 & 0.065229 & 0.400456 & -0.123046 \\
\hline & & & & 1 & -0.559002 & 0.262375 & -1.004014 \\
\hline \multirow{2}{*}{\multicolumn{4}{|c|}{ 25. TS-2't (-1018.82189) }} & 6 & -1.156003 & -0.651758 & 1.227849 \\
\hline & & & & 6 & -0.172038 & -1.188492 & 2.239483 \\
\hline 6 & -2.241904 & -1.977748 & -1.635710 & 1 & -0.724174 & -1.595642 & 3.096854 \\
\hline 1 & -2.923484 & -1.257397 & -1.180524 & 1 & 0.436526 & -1.995844 & 1.819861 \\
\hline 1 & -1.939437 & -1.632533 & -2.625956 & 1 & 0.505894 & -0.415590 & 2.616551 \\
\hline 16 & -0.698158 & -2.119347 & -0.635157 & 6 & -1.936595 & -1.532554 & 0.460095 \\
\hline 6 & -1.410453 & -2.855545 & 0.880445 & 1 & -1.627323 & -2.568566 & 0.345502 \\
\hline 1 & -2.205129 & -2.240576 & 1.304896 & 6 & -3.082133 & -1.056514 & -0.232592 \\
\hline 1 & -0.595531 & -2.967480 & 1.596052 & 6 & -3.955733 & -2.062584 & -0.977362 \\
\hline 6 & 0.006671 & -0.513809 & -0.241115 & 1 & -3.585140 & -3.090254 & -0.905662 \\
\hline 1 & 0.790955 & -0.459336 & -0.998982 & 1 & -4.974522 & -2.025276 & -0.573516 \\
\hline 6 & 1.328868 & -0.526485 & 1.211928 & 1 & -4.024727 & -1.780521 & -2.035073 \\
\hline 6 & 0.585621 & -0.315140 & 2.516001 & 8 & -3.427701 & 0.153049 & -0.271904 \\
\hline 1 & 1.274985 & -0.465950 & 3.356308 & 1 & 0.874004 & 4.190984 & -0.688102 \\
\hline 1 & 0.196970 & 0.706213 & 2.585631 & 1 & -1.933544 & 3.459120 & 0.522345 \\
\hline 1 & -0.257830 & -1.000440 & 2.653873 & 6 & 1.401594 & -0.209498 & -0.225186 \\
\hline 6 & 2.304902 & 0.435365 & 0.846842 & 6 & 1.561276 & -1.321916 & -1.079202 \\
\hline 1 & 2.310041 & 1.408977 & 1.330405 & 6 & 2.774093 & -2.002648 & -1.157856 \\
\hline 6 & 3.151554 & 0.176740 & -0.262868 & 6 & 3.873232 & -1.587595 & -0.402538 \\
\hline 6 & 4.266512 & 1.169679 & -0.589999 & 6 & 3.735102 & -0.486560 & 0.445830 \\
\hline 1 & 4.285812 & 2.033278 & 0.083511 & 6 & 2.519752 & 0.188316 & 0.539313 \\
\hline 1 & 5.234450 & 0.657227 & -0.530795 & 1 & 0.715198 & -1.650927 & -1.676649 \\
\hline
\end{tabular}




$\begin{array}{rrrr}1 & 2.863226 & -2.857883 & -1.823261 \\ 1 & 4.822292 & -2.111043 & -0.475329 \\ 1 & 4.578636 & -0.151112 & 1.044259 \\ 1 & 2.438471 & 1.027945 & 1.227472 \\ 1 & -1.691145 & 0.237396 & 1.557721\end{array}$

$\mathrm{E}(\mathrm{MeCN})=-1019.0408915$

NImag $=1(-277.6)$

27. I-2't conformer 1(-1018.84608)

$\begin{array}{crrr}6 & -1.996209 & 2.477093 & 0.051774 \\ 1 & -2.126265 & 3.332689 & -0.615230 \\ 1 & -2.478457 & 1.548168 & -0.320609 \\ 16 & -0.193392 & 2.136050 & 0.149177 \\ 6 & 0.304792 & 2.566018 & -1.552457 \\ 1 & -0.339752 & 2.030278 & -2.254278 \\ 1 & 1.344980 & 2.262147 & -1.677804 \\ 6 & -0.148484 & 0.361140 & 0.106332 \\ 1 & -0.775382 & 0.126081 & -0.762991 \\ 6 & -0.958674 & -0.273817 & 1.324244 \\ 6 & -0.096924 & -0.539016 & 2.561216 \\ 1 & -0.736527 & -0.912722 & 3.368130 \\ 1 & 0.665579 & -1.298623 & 2.360919 \\ 1 & 0.412181 & 0.362841 & 2.926344 \\ 6 & -1.648466 & -1.494330 & 0.791366 \\ 1 & -1.442591 & -2.454018 & 1.255257 \\ 6 & -2.525716 & -1.371711 & -0.269377 \\ 6 & -3.233406 & -2.611860 & -0.804474 \\ 1 & -2.963204 & -3.526095 & -0.264025 \\ 1 & -4.320207 & -2.473010 & -0.739229 \\ 1 & -2.999394 & -2.746030 & -1.868742 \\ 8 & -2.778279 & -0.261164 & -0.882771 \\ 1 & 0.207978 & 3.646646 & -1.677395 \\ 1 & -2.337249 & 2.736679 & 1.054592 \\ 6 & 1.263765 & -0.144044 & -0.081217 \\ 6 & 1.440468 & -1.293876 & -0.867890 \\ 6 & 2.708687 & -1.850374 & -1.037486 \\ 6 & 3.824092 & -1.258190 & -0.442983 \\ 6 & 3.661078 & -0.112665 & 0.337991 \\ 6 & 2.390362 & 0.433568 & 0.525420 \\ 1 & 0.569717 & -1.757421 & -1.320784 \\ 1 & 2.824458 & -2.745568 & -1.642641 \\ 1 & 4.812724 & -1.686179 & -0.584999 \\ 1 & 4.521084 & 0.351962 & 0.813009 \\ 1 & 2.282429 & 1.308830 & 1.162382 \\ 1 & -1.713779 & 0.478246 & 1.608670\end{array}$

$\mathrm{E}(\mathrm{MeCN})=-1019.0597942$

NImag $=0$
28. I-2' $t$ conformer2 (Optimized in $\mathrm{MeCN}$ at the B3LYP/6-31G* level)

$\begin{array}{crrc}6 & -1.753010 & 2.714405 & -0.188902 \\ 1 & -1.775172 & 3.542278 & -0.901730 \\ 1 & -2.287129 & 1.827847 & -0.559803 \\ 16 & -0.000308 & 2.265931 & 0.100699 \\ 6 & 0.713737 & 2.564641 & -1.549239 \\ 1 & 0.134698 & 2.031983 & -2.308306 \\ 1 & 1.743919 & 2.205202 & -1.530966 \\ 6 & -0.162084 & 0.317882 & 0.091276 \\ 1 & -0.738582 & 0.190759 & -0.826590 \\ 6 & -1.076410 & -0.156386 & 1.256611 \\ 6 & -0.389940 & -0.286016 & 2.618546 \\ 1 & -1.132342 & -0.586976 & 3.365972 \\ 1 & 0.395326 & -1.049552 & 2.604036 \\ 1 & 0.061556 & 0.655653 & 2.956216 \\ 6 & -1.688197 & -1.434671 & 0.752006 \\ 1 & -1.305100 & -2.382520 & 1.121921 \\ 6 & -2.641305 & -1.379788 & -0.249482 \\ 6 & -3.222975 & -2.690694 & -0.781667 \\ 1 & -2.826730 & -3.575466 & -0.270844 \\ 1 & -4.316523 & -2.686962 & -0.676629 \\ 1 & -3.014360 & -2.786136 & -1.856189 \\ 8 & -3.082236 & -0.291072 & -0.783556 \\ 1 & 0.697482 & 3.641714 & -1.733202 \\ 1 & -2.168189 & 3.042351 & 0.765965 \\ 6 & 1.219719 & -0.268987 & -0.043702 \\ 6 & 1.451852 & -1.205982 & -1.063251 \\ 6 & 2.703663 & -1.805875 & -1.211305 \\ 6 & 3.750912 & -1.461785 & -0.353560 \\ 6 & 3.537763 & -0.516686 & 0.653354 \\ 6 & 2.281714 & 0.072567 & 0.810667 \\ 1 & 0.640691 & -1.472499 & -1.736327 \\ 1 & 2.861078 & -2.536721 & -2.003051 \\ 1 & 4.730399 & -1.922480 & -0.472422 \\ 1 & 4.350032 & -0.236700 & 1.322241 \\ 1 & 2.136849 & 0.804892 & 1.602401 \\ 1 & -1.877718 & 0.591264 & 1.346449\end{array}$

$\mathrm{E}(\mathrm{MeCN})=-1019.0636208$

NImag $=0$

29. TS-2'R (-1018.84922)

$\begin{array}{cccc}16 & -0.299881 & 1.964527 & 0.264296 \\ 6 & -0.025155 & 0.077584 & 0.114793 \\ 6 & -0.753359 & -0.739097 & 1.256635 \\ 6 & -1.846561 & -1.564942 & 0.620007 \\ 6 & -2.696553 & -1.107806 & -0.362734\end{array}$




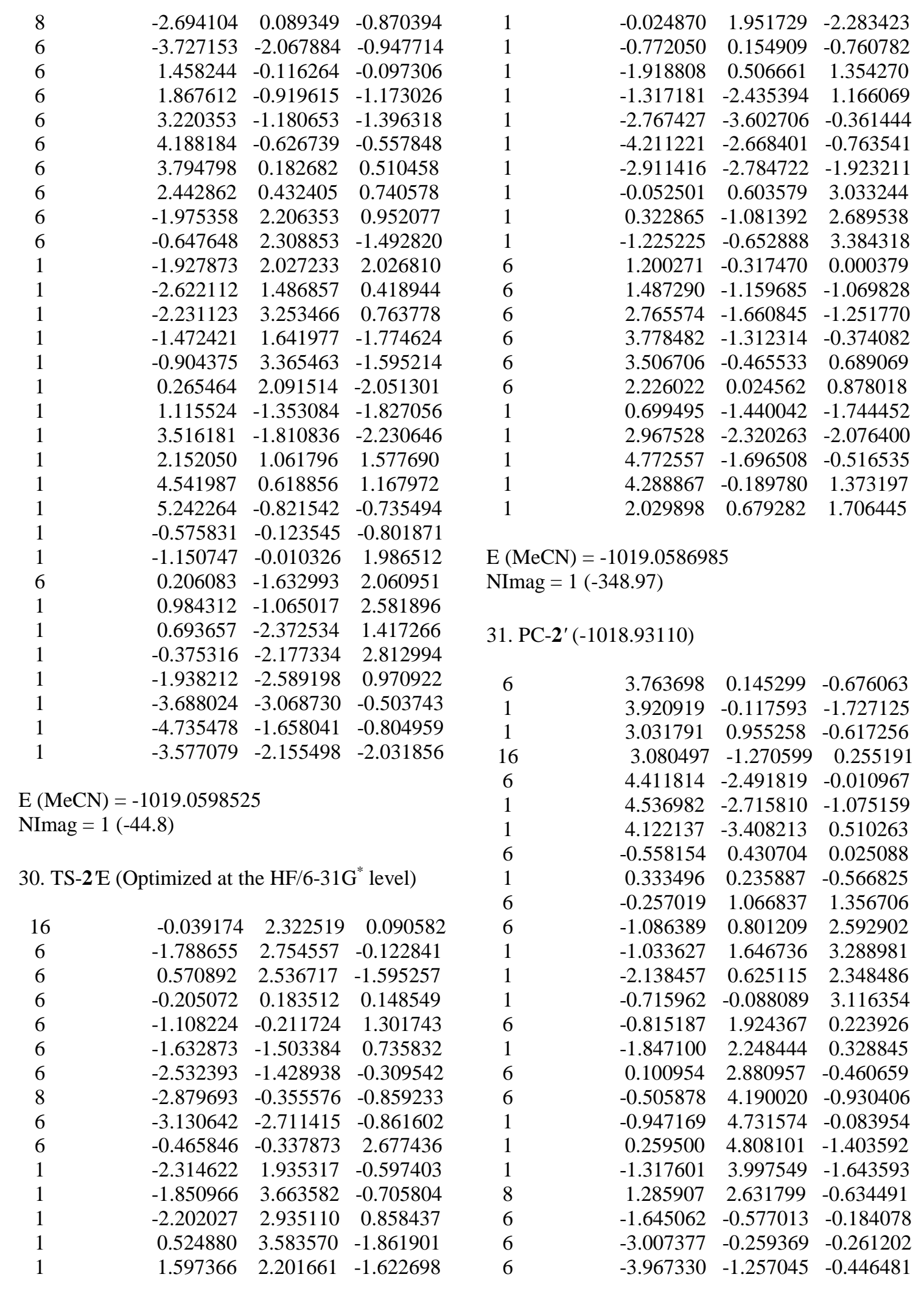




$\begin{array}{lrrr}6 & -3.581500 & -2.591673 & -0.565548 \\ 6 & -2.225396 & -2.920985 & -0.501246 \\ 6 & -1.269314 & -1.924743 & -0.313787 \\ 1 & -3.329839 & 0.775595 & -0.191099 \\ 1 & -5.018371 & -0.985572 & -0.504496 \\ 1 & -4.328274 & -3.367389 & -0.712332 \\ 1 & -1.910606 & -3.956837 & -0.599208 \\ 1 & -0.214214 & -2.184511 & -0.266440 \\ 1 & 0.803767 & 1.230587 & 1.537139 \\ 1 & 5.361620 & -2.137464 & 0.402087 \\ 1 & 4.708660 & 0.481349 & -0.237322\end{array}$

$\mathrm{E}(\mathrm{MeCN})=-1019.1242485$

NImag $=0$

\section{TS-3c (-1015.66131)}

$\begin{array}{crrr}6 & 0.796437 & 2.748014 & -0.907930 \\ 1 & 1.833217 & 2.560950 & -0.624166 \\ 1 & 0.648922 & 2.598354 & -1.979709 \\ 16 & -0.291014 & 1.552050 & -0.054690 \\ 6 & 0.142427 & 1.900447 & 1.680773 \\ 1 & 1.204211 & 1.703124 & 1.827926 \\ 1 & -0.480910 & 1.246341 & 2.290077 \\ 6 & 0.413480 & -0.045598 & -0.410717 \\ 1 & -0.036997 & -0.362305 & -1.351650 \\ 6 & -0.666503 & -1.352255 & 0.618638 \\ 6 & 0.086373 & -1.713141 & 1.882853 \\ 1 & -0.024958 & -0.960585 & 2.671539 \\ 1 & -0.322065 & -2.653052 & 2.275955 \\ 1 & 1.154394 & -1.868714 & 1.702735 \\ 1 & -0.514431 & -2.076083 & -0.181315 \\ 6 & -1.996407 & -0.862376 & 0.716238 \\ 1 & -2.395600 & -0.555712 & 1.680977 \\ 6 & -2.760183 & -0.700283 & -0.472523 \\ 6 & -4.223947 & -0.277586 & -0.357454 \\ 1 & -4.541458 & -0.096476 & 0.675171 \\ 1 & -4.396495 & 0.627958 & -0.951173 \\ 1 & -4.859102 & -1.062190 & -0.785777 \\ 8 & -2.276448 & -0.871153 & -1.617504 \\ 6 & 1.854066 & -0.174876 & -0.255015 \\ 8 & 2.610467 & 0.572496 & 0.369343 \\ 8 & 2.287929 & -1.324581 & -0.837712 \\ 6 & 3.681899 & -1.614622 & -0.667324 \\ 1 & 4.299431 & -0.827469 & -1.109298 \\ 1 & 3.935195 & -1.707066 & 0.392589 \\ 1 & 3.846271 & -2.561724 & -1.182601 \\ 1 & -0.112605 & 2.944674 & 1.876929 \\ 1 & 0.477771 & 3.756743 & -0.631415\end{array}$

$\mathrm{E}(\mathrm{MeCN})=-1019.88310975$

NImag $=1(-367.1)$
33. TS-3 $c$ conformer2 (-1015.6640743)

$\begin{array}{crrr}16 & 0.313326 & 1.622099 & -0.163162 \\ 6 & -0.686581 & 0.352239 & 0.622633 \\ 6 & -1.686873 & -0.105755 & -0.345594 \\ 8 & -1.603658 & 0.027915 & -1.560587 \\ 8 & -2.714300 & -0.756380 & 0.257363 \\ 6 & -3.693776 & -1.307476 & -0.636032 \\ 6 & 0.519690 & -1.171573 & 1.097046 \\ 6 & 1.281584 & -1.660148 & 0.009186 \\ 6 & 2.440823 & -0.968204 & -0.401805 \\ 8 & 2.793936 & 0.144954 & 0.083227 \\ 6 & 3.321171 & -1.574485 & -1.488742 \\ 6 & -0.428347 & -2.119254 & 1.808172 \\ 1 & -1.089083 & 0.692576 & 1.577127 \\ 1 & -4.155323 & -0.519665 & -1.238208 \\ 1 & -3.238276 & -2.041524 & -1.305759 \\ 1 & -4.436830 & -1.783406 & 0.004985 \\ 1 & -1.027503 & -2.699780 & 1.100316 \\ 1 & -1.116294 & -1.607277 & 2.489063 \\ 1 & 0.167070 & -2.826854 & 2.398751 \\ 1 & 1.099121 & -0.554966 & 1.784683 \\ 1 & 0.945751 & -2.539411 & -0.534149 \\ 1 & 4.340636 & -1.695260 & -1.103249 \\ 1 & 3.383417 & -0.885451 & -2.339856 \\ 1 & 2.958350 & -2.544601 & -1.843334 \\ 6 & -0.879999 & 2.989016 & -0.476411 \\ 6 & 1.219113 & 2.333669 & 1.244089 \\ 1 & -0.323545 & 3.850841 & -0.856061 \\ 1 & -1.420940 & 3.250627 & 0.436531 \\ 1 & -1.564284 & 2.621094 & -1.242015 \\ 1 & 1.660323 & 3.272993 & 0.902360 \\ 1 & 0.549297 & 2.508673 & 2.089666 \\ 1 & 2.006248 & 1.607734 & 1.454465\end{array}$

$\mathrm{E}(\mathrm{MeCN})=-1015.8860738$

NImag $=1(-322.0)$

34. I-3c conformer 1 (-1015.677948)

$\begin{array}{cccc}16 & -1.150021 & -1.256505 & -.397091 \\ 6 & -.583529 & -3.039999 & -.366764 \\ 6 & -2.476364 & -1.482655 & .836349 \\ 6 & .292648 & -.596216 & .608336 \\ 6 & -.028926 & .728394 & 1.370924 \\ 6 & .969841 & .962451 & 2.516690 \\ 6 & -.155621 & 1.898224 & .435711 \\ 6 & -1.220856 & 1.901016 & -.420848 \\ 8 & -2.060535 & .904476 & -.474628 \\ 6 & -1.491081 & 3.060642 & -1.360811 \\ 1 & .328360 & -3.096938 & -.963058\end{array}$




\begin{tabular}{|c|c|c|c|c|c|c|c|}
\hline 1 & -1.363216 & -3.637080 & -.848588 & 6 & 1.801030 & -0.063120 & -0.502399 \\
\hline 1 & -.406127 & -3.409341 & .648371 & 8 & 2.380184 & 0.975605 & -0.760817 \\
\hline 1 & -3.121895 & -2.282110 & .463357 & 8 & 2.423226 & -1.251698 & -0.450639 \\
\hline 1 & -2.060964 & -1.765720 & 1.807138 & 6 & 3.832893 & -1.230411 & -0.749689 \\
\hline 1 & -2.999317 & -.530097 & .876403 & 1 & 4.157559 & -2.268019 & -0.676972 \\
\hline 1 & .535174 & -1.398514 & 1.319307 & 1 & 4.001773 & -0.840009 & -1.756132 \\
\hline 1 & 1.052454 & .090890 & 3.179368 & 1 & 4.365570 & -0.605695 & -0.028653 \\
\hline 1 & 1.968505 & 1.202929 & 2.145628 & & & & \\
\hline 1 & .617554 & 1.809114 & 3.115536 & \multicolumn{4}{|c|}{$E(\mathrm{MeCN})=-1019.8983435$} \\
\hline 1 & -1.006075 & .540350 & 1.843273 & \multicolumn{4}{|c|}{ NImag $=0$} \\
\hline 1 & .551692 & 2.719020 & .499870 & & & & \\
\hline 1 & -1.478451 & 2.712489 & -2.401325 & \multirow{2}{*}{\multicolumn{4}{|c|}{ 36. I-3c conformer 3 (-1015.6762077) }} \\
\hline 1 & -2.494584 & 3.463545 & -1.174439 & & & & \\
\hline 1 & -.761431 & 3.869998 & -1.248592 & 16 & -0.372149 & 1.415264 & 0.654468 \\
\hline 6 & 1.428767 & -.475638 & -.407061 & 6 & 1.012754 & 2.600271 & 0.899790 \\
\hline 8 & 1.343532 & -.779934 & -1.579250 & 6 & -1.233130 & 2.192396 & -0.755209 \\
\hline 8 & 2.558637 & -.046081 & .170375 & 6 & 0.345232 & -0.142259 & -0.107101 \\
\hline 6 & 3.674130 & .144131 & -.722047 & 6 & -0.247151 & -1.313790 & 0.712214 \\
\hline 1 & 4.491968 & .491203 & -.090865 & 6 & 0.306666 & -1.432950 & 2.136655 \\
\hline 1 & 3.934896 & -.796361 & -1.213510 & 6 & -1.749823 & -1.167368 & 0.681353 \\
\hline \multirow[t]{2}{*}{1} & 3.424677 & .890921 & -1.479260 & 6 & -2.451259 & -0.888533 & -0.480409 \\
\hline & & & & 8 & -1.909040 & -0.609113 & -1.621454 \\
\hline \multicolumn{4}{|c|}{$E(\mathrm{MeCN})=-1015.8991136$} & 6 & -3.974267 & -0.841177 & -0.433883 \\
\hline \multirow{2}{*}{\multicolumn{4}{|c|}{ NImag $=0$}} & 1 & 1.661639 & 2.195829 & 1.679703 \\
\hline & & & & 1 & 0.546095 & 3.521703 & 1.258084 \\
\hline \multirow{2}{*}{\multicolumn{4}{|c|}{ 35. I-3c conformer 2(-1015.67987) }} & 1 & 1.578790 & 2.752708 & -0.016604 \\
\hline & & & & 1 & -1.960901 & 2.899606 & -0.352599 \\
\hline 16 & -0.508647 & 1.254858 & 0.547237 & 1 & -0.504336 & 2.695419 & -1.394570 \\
\hline 6 & 0.859608 & 1.855230 & 1.672059 & 1 & -1.721719 & 1.366410 & -1.300028 \\
\hline 6 & -0.400540 & 2.626580 & -0.652288 & 1 & -0.257207 & -0.211268 & -1.055768 \\
\hline 6 & 0.291921 & -0.192398 & -0.319966 & 1 & 1.395560 & -1.550475 & 2.142243 \\
\hline 6 & -0.268429 & -1.497677 & 0.288948 & 1 & 0.042035 & -0.557812 & 2.745103 \\
\hline 6 & 0.189241 & -1.746121 & 1.742090 & 1 & -0.130972 & -2.307368 & 2.631217 \\
\hline 6 & -1.773148 & -1.567926 & 0.142690 & 1 & 0.074725 & -2.201900 & 0.145085 \\
\hline 6 & -2.582044 & -0.622068 & -0.424827 & 1 & -2.292153 & -1.401472 & 1.592749 \\
\hline 8 & -2.190279 & 0.552524 & -0.859611 & 1 & -4.333412 & 0.154044 & -0.727828 \\
\hline 6 & -4.070763 & -0.889995 & -0.569074 & 1 & -4.380132 & -1.547479 & -1.168271 \\
\hline 1 & 1.090721 & 1.050693 & 2.374155 & 1 & -4.381761 & -1.086396 & 0.552787 \\
\hline 1 & 0.437942 & 2.697074 & 2.229296 & 6 & 1.808167 & -0.090380 & -0.465403 \\
\hline 1 & 1.755390 & 2.160026 & 1.131490 & 8 & 2.398740 & 0.879943 & -0.906079 \\
\hline 1 & -0.577931 & 3.549337 & -0.093703 & 8 & 2.386335 & -1.299215 & -0.329912 \\
\hline 1 & 0.591271 & 2.637316 & -1.104790 & 6 & 3.745840 & -1.392097 & -0.793523 \\
\hline 1 & -1.195127 & 2.445709 & -1.372870 & 1 & 4.031928 & -2.432322 & -0.638883 \\
\hline 1 & -0.150173 & -0.074333 & -1.313603 & 1 & 3.807092 & -1.127236 & -1.851924 \\
\hline 1 & 1.281278 & -1.750281 & 1.845645 & 1 & 4.392669 & -0.723825 & -0.21933 \\
\hline 1 & -0.238397 & -1.001957 & 2.424908 & \multirow{2}{*}{\multicolumn{4}{|c|}{$E(\mathrm{MeCN})=-1015.8969819$}} \\
\hline 1 & -0.179987 & -2.722131 & 2.075041 & & & & \\
\hline 1 & 0.198224 & -2.289494 & -0.320428 & \multicolumn{4}{|c|}{ NImag $=0$} \\
\hline 1 & -2.218371 & -2.498525 & 0.485866 & & & & \\
\hline 1 & -4.641023 & -0.128100 & -0.022198 & & & & \\
\hline 1 & -4.360899 & -0.802643 & -1.623917 & & & & \\
\hline 1 & -4.363142 & -1.880713 & -0.205658 & & & & \\
\hline
\end{tabular}


37. I-3 $c$ conformer 4 (-1015.6761385)

$\begin{array}{crrr}16 & 0.097427 & 1.656348 & 0.634647 \\ 6 & 0.253629 & 2.620078 & -0.909684 \\ 6 & -1.713250 & 1.783409 & 0.824131 \\ 6 & 0.417576 & -0.074611 & -0.020709 \\ 6 & -0.226027 & -1.230260 & 0.788816 \\ 6 & 0.064909 & -1.163244 & 2.300028 \\ 6 & -1.688635 & -1.411834 & 0.456259 \\ 6 & -2.323212 & -0.913793 & -0.657977 \\ 8 & -1.824666 & -0.066567 & -1.519403 \\ 6 & -3.772074 & -1.308491 & -0.914358 \\ 1 & 1.306855 & 2.614616 & -1.186038 \\ 1 & -0.084222 & 3.637713 & -0.700383 \\ 1 & -0.376339 & 2.141190 & -1.663720 \\ 1 & -1.911590 & 2.830840 & 1.068667 \\ 1 & -2.162811 & 1.462843 & -0.122852 \\ 1 & -2.022479 & 1.124467 & 1.632960 \\ 1 & -0.164344 & -0.073316 & -0.986466 \\ 1 & 1.141299 & -1.111151 & 2.510551 \\ 1 & -0.419977 & -0.302070 & 2.777798 \\ 1 & -0.328677 & -2.059792 & 2.790840 \\ 1 & 0.344967 & -2.095516 & 0.410779 \\ 1 & -2.214107 & -2.129930 & 1.080960 \\ 1 & -4.410001 & -0.414192 & -0.926886 \\ 1 & -3.855086 & -1.762459 & -1.909756 \\ 1 & -4.164835 & -2.011702 & -0.171790 \\ 6 & 1.913199 & -0.112742 & -0.200279 \\ 8 & 2.645651 & 0.862256 & -0.123404 \\ 8 & 2.349303 & -1.338938 & -0.522534 \\ 6 & 3.758042 & -1.446686 & -0.802322 \\ 1 & 3.923129 & -2.496755 & -1.042296 \\ 1 & 4.027602 & -0.809892 & -1.648501 \\ 1 & 4.344517 & -1.151433 & 0.071083\end{array}$

$\mathrm{E}(\mathrm{MeCN})=-1015.8965228$

NImag $=0$

38. I-3c conformer 5 (-1015.6746847)

$\begin{array}{cccc}16 & .791727 & 1.568116 & .603145 \\ 6 & 1.228839 & 1.881137 & -1.153607 \\ 6 & -.363194 & 3.026885 & .791209 \\ 6 & -.540350 & .255395 & .808967 \\ 6 & .086761 & -1.087930 & 1.289474 \\ 6 & -.919915 & -1.954822 & 2.062178 \\ 6 & .786044 & -1.817787 & .176649 \\ 6 & 1.935472 & -1.255855 & -.299247 \\ 8 & 2.355018 & -.089785 & .117459 \\ 6 & 2.802315 & -1.948553 & -1.334909 \\ 1 & .975045 & 1.003145 & -1.738743 \\ 1 & 2.307992 & 2.020705 & -1.149087\end{array}$

$\begin{array}{lccc}1 & .684423 & 2.753961 & -1.515748 \\ 1 & .236917 & 3.930120 & .649289 \\ 1 & -1.177595 & 2.996188 & .064846 \\ 1 & -.753030 & 3.023690 & 1.812968 \\ 1 & -1.128029 & .695797 & 1.627360 \\ 1 & -1.438470 & -1.387720 & 2.846599 \\ 1 & -1.674290 & -2.393625 & 1.405752 \\ 1 & -.373059 & -2.772172 & 2.544390 \\ 1 & .849482 & -.762955 & 2.015647 \\ 1 & .428846 & -2.791186 & -.144235 \\ 1 & 2.906086 & -1.323756 & -2.231872 \\ 1 & 3.813418 & -2.090761 & -.933330 \\ 1 & 2.401928 & -2.924611 & -1.629352 \\ 6 & -1.464752 & .176661 & -.407989 \\ 8 & -1.462284 & .928328 & -1.361374 \\ 8 & -2.360568 & -.808887 & -.256009 \\ 6 & -3.289149 & -.977671 & -1.344350 \\ 1 & -3.931672 & -1.806357 & -1.047673 \\ 1 & -3.873935 & -.066569 & -1.492935 \\ 1 & -2.751449 & -1.215762 & -2.265044\end{array}$

$\mathrm{E}(\mathrm{MeCN})=-1015.8932651$

NImag $=0$

39. TS-3t (-1015.65647)

$\begin{array}{crrr}6 & -2.513139 & -0.706783 & 1.479526 \\ 16 & -2.440049 & -0.469488 & -0.331962 \\ 6 & -3.406939 & 1.101795 & -0.448179 \\ 6 & -0.672204 & -0.204754 & -0.653325 \\ 6 & 0.405831 & -1.570083 & 0.001255 \\ 6 & -0.250894 & -2.862832 & -0.468738 \\ 6 & 1.755840 & -1.374016 & -0.416843 \\ 6 & 2.615746 & -0.534187 & 0.329476 \\ 8 & 2.240383 & 0.139448 & 1.322247 \\ 6 & 4.082931 & -0.441180 & -0.092765 \\ 1 & -1.989884 & -1.634470 & 1.712075 \\ 1 & -3.566657 & -0.794307 & 1.754210 \\ 1 & -2.029182 & 0.145287 & 1.957221 \\ 1 & -4.413565 & 0.902953 & -0.069553 \\ 1 & -2.898795 & 1.870802 & 0.133974 \\ 1 & -3.453713 & 1.369169 & -1.505604 \\ 1 & -0.553466 & -0.312237 & -1.730593 \\ 1 & 0.286242 & -1.372450 & 1.068732 \\ 1 & -1.311876 & -2.953198 & -0.199167 \\ 1 & 0.276029 & -3.711948 & -0.019697 \\ 1 & -0.176441 & -2.971064 & -1.557518 \\ 1 & 2.102543 & -1.840372 & -1.336259 \\ 1 & 4.342550 & -1.122079 & -0.910575 \\ 1 & 4.721150 & -0.660904 & 0.770888 \\ 1 & 4.315152 & 0.585182 & -0.405035\end{array}$




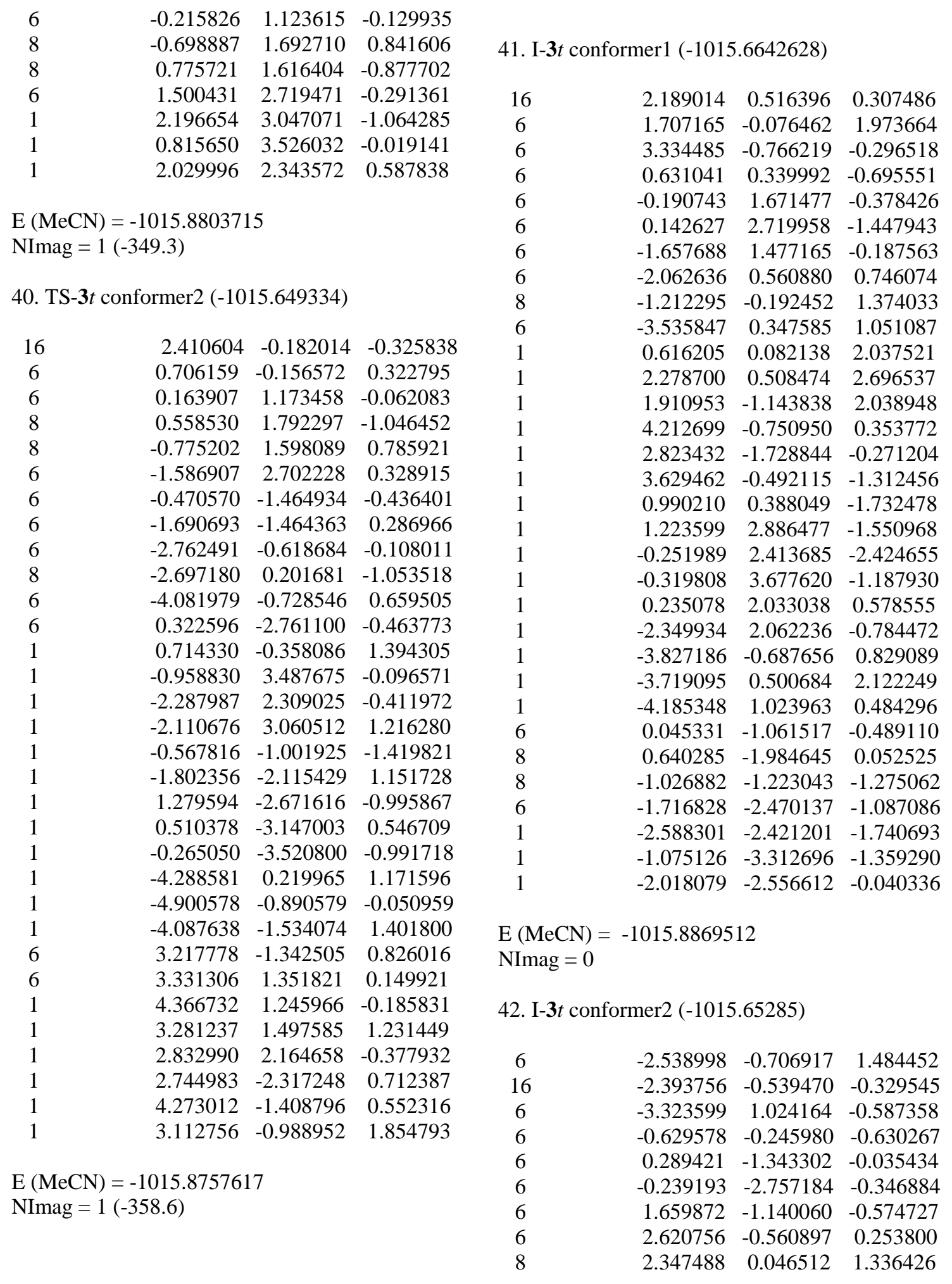




$\begin{array}{lrrr}6 & 4.087963 & -0.618503 & -0.177031 \\ 1 & -1.986947 & -1.601036 & 1.774912 \\ 1 & -3.600392 & -0.837086 & 1.707117 \\ 1 & -2.116540 & 0.181031 & 1.953245 \\ 1 & -4.370584 & 0.835655 & -0.335902 \\ 1 & -2.892733 & 1.806614 & 0.037625 \\ 1 & -3.241394 & 1.275826 & -1.647038 \\ 1 & -0.554030 & -0.273223 & -1.720854 \\ 1 & 0.334983 & -1.169955 & 1.047111 \\ 1 & -1.246495 & -2.974721 & 0.042981 \\ 1 & 0.449608 & -3.485253 & 0.090042 \\ 1 & -0.264852 & -2.936199 & -1.429545 \\ 1 & 1.923472 & -1.637680 & -1.505324 \\ 1 & 4.241765 & -1.141544 & -1.127949 \\ 1 & 4.673137 & -1.124221 & 0.601217 \\ 1 & 4.493171 & 0.398105 & -0.262659 \\ 6 & -0.226682 & 1.133323 & -0.079102 \\ 8 & -0.716226 & 1.621319 & 0.926741 \\ 8 & 0.698132 & 1.707761 & -0.830369 \\ 6 & 1.452170 & 2.766167 & -0.185377 \\ 1 & 2.117166 & 3.150597 & -0.958475 \\ 1 & 0.780938 & 3.547945 & 0.177244 \\ 1 & 2.011878 & 2.307057 & 0.634157\end{array}$

$\mathrm{E}(\mathrm{MeCN})=-1015.8844918$ NImag $=0$

43. I-3c conformer6(-1015.6685403)

$\begin{array}{crrc}6 & 3.089578 & -1.344252 & -0.252233 \\ 1 & 3.029369 & -0.713803 & -1.139055 \\ 1 & 3.718480 & -0.888050 & 0.516153 \\ 16 & 1.418534 & -1.578793 & 0.444852 \\ 6 & 0.439490 & -2.197330 & -0.954382 \\ 1 & 0.650906 & -1.591964 & -1.834826 \\ 1 & -0.644623 & -2.130659 & -0.643822 \\ 6 & 0.822023 & 0.130952 & 0.818509 \\ 1 & 1.521841 & 0.452209 & 1.602633 \\ 6 & -0.671081 & 0.078997 & 1.366018 \\ 6 & -0.751566 & 0.954060 & 2.629809 \\ 1 & -0.565847 & 2.007164 & 2.390078 \\ 1 & -1.758873 & 0.878358 & 3.049269 \\ 1 & -0.032907 & 0.647278 & 3.402700 \\ 1 & -0.866823 & -0.966171 & 1.648038 \\ 6 & -1.686396 & 0.440470 & 0.324537 \\ 1 & -1.960850 & 1.487877 & 0.227212 \\ 6 & -2.445147 & -0.544779 & -0.286684 \\ 6 & -3.595725 & -0.126480 & -1.199184 \\ 1 & -3.742132 & 0.958857 & -1.242605 \\ 1 & -3.419683 & -0.500231 & -2.216341 \\ 1 & -4.523637 & -0.595671 & -0.849693 \\ 8 & -2.249078 & -1.808071 & -0.159424 \\ 6 & 0.953838 & 1.072292 & -0.376804 \\ 8 & 1.246631 & 0.762561 & -1.514234 \\ 1 & 0.744477 & -3.239550 & -1.083673\end{array}$

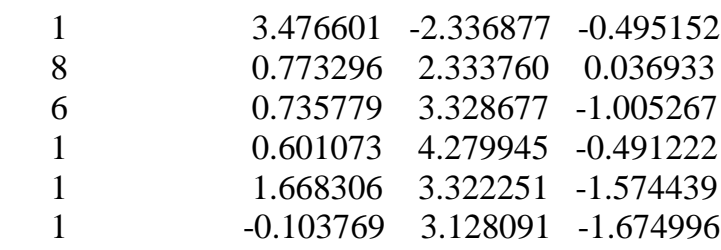

$\mathrm{E}(\mathrm{MeCN})=-1015.8958163$

$\mathrm{NImag}=0$

44. TS-3R (Optimized at the mPW1PW91/6-31G* level)

$\begin{array}{crrr}6 & 1.747383 & -0.591388 & 2.238803 \\ 1 & 1.993775 & 0.448821 & 2.022843 \\ 1 & 0.903537 & -0.639266 & 2.928336 \\ 16 & 1.228473 & -1.425660 & 0.721473 \\ 6 & 2.740134 & -1.297812 & -0.265954 \\ 1 & 3.153034 & -0.290036 & -0.207707 \\ 1 & 2.476638 & -1.525922 & -1.298410 \\ 6 & 0.017590 & -0.201400 & -0.015033 \\ 1 & -0.490853 & 0.199247 & 0.864582 \\ 6 & -1.014809 & -0.897700 & -0.955548 \\ 6 & -0.620296 & -2.279025 & -1.470727 \\ 1 & -0.581782 & -3.027588 & -0.671401 \\ 1 & -1.386560 & -2.612039 & -2.176180 \\ 1 & 0.333412 & -2.280313 & -2.013170 \\ 1 & -1.029301 & -0.227439 & -1.826991 \\ 6 & -2.335240 & -0.888972 & -0.255940 \\ 1 & -2.960700 & -1.774634 & -0.246349 \\ 6 & -2.727299 & 0.333621 & 0.238515 \\ 6 & -4.081095 & 0.507051 & 0.898307 \\ 1 & -4.661397 & -0.420309 & 0.921887 \\ 1 & -3.955379 & 0.874689 & 1.923144 \\ 1 & -4.656911 & 1.267990 & 0.360292 \\ 8 & -1.955558 & 1.360832 & 0.194598 \\ 6 & 0.779630 & 0.945807 & -0.647071 \\ 8 & 1.069361 & 1.021229 & -1.816513 \\ 1 & 3.454878 & -2.031973 & 0.109211 \\ 1 & 2.597741 & -1.129731 & 2.661116 \\ 8 & 1.156364 & 1.850789 & 0.272325 \\ 6 & 1.727542 & 3.048086 & -0.259216 \\ 1 & 0.999579 & 3.549897 & -0.897894 \\ 1 & 1.966230 & 3.667818 & 0.603258 \\ 1 & 2.625921 & 2.828947 & -0.840235 \\ \mathrm{E}(\mathrm{MeCN})=-1015.8805001 & \\ \mathrm{NImag}=1 & (-80.2) & & \\ & & & \\ & & & \\ 1 & & & \\ 1 & & & \\ 1 & & & \\ 1 & & & \\ 1 & & & \end{array}$

45. TS-3E (-1015.66247)

$\begin{array}{cccc}16 & 2.292893 & -0.737977 & -0.050478 \\ 6 & 1.631203 & -1.149306 & -1.717092 \\ 6 & 3.185352 & 0.824225 & -0.357448 \\ 6 & 0.523195 & -0.163841 & 0.888978 \\ 6 & -0.406925 & -1.376613 & 0.917916\end{array}$




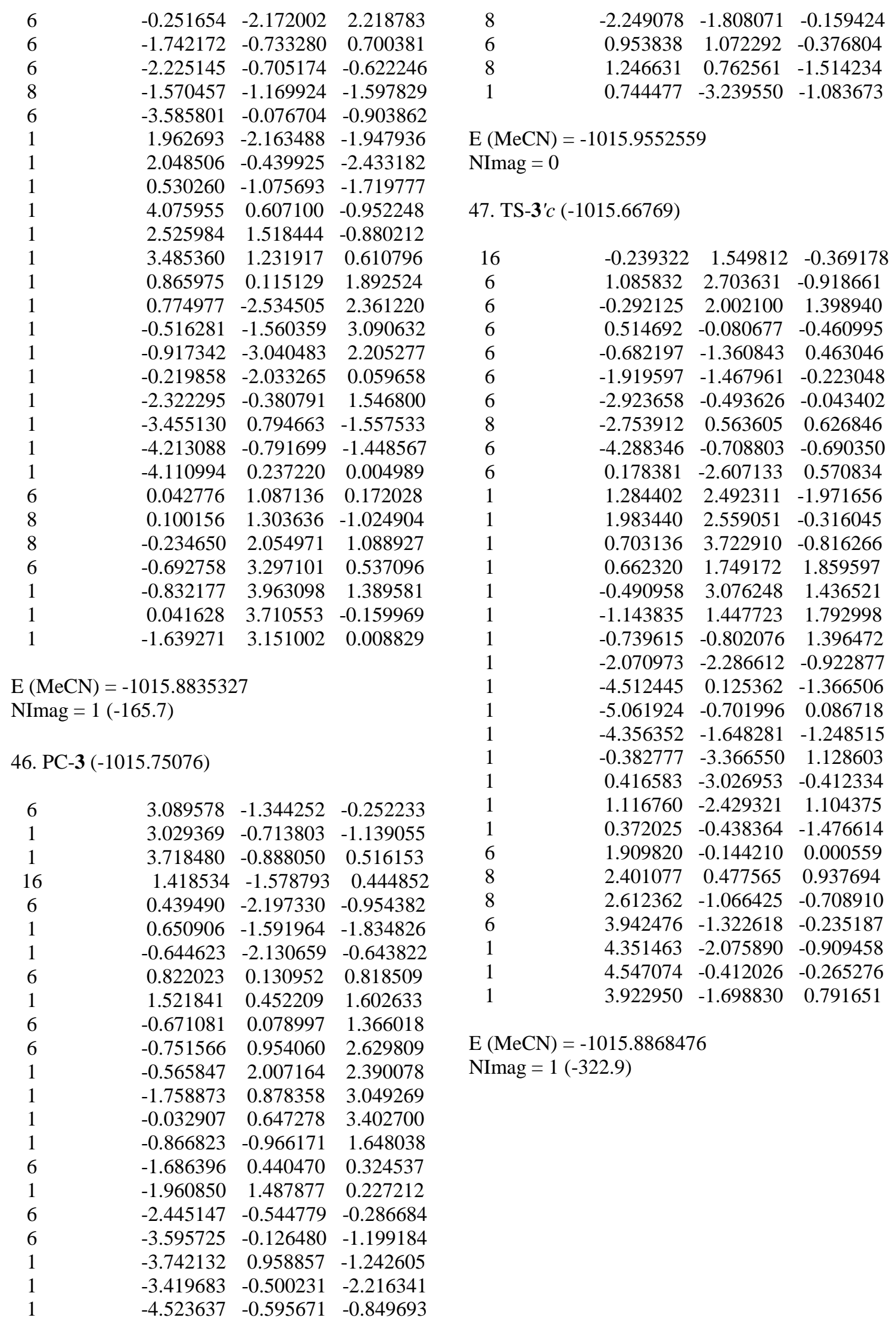


48. TS-3'c conformer2 (-1015.660134)

$\begin{array}{crrr}16 & 0.087622 & 1.551257 & -0.260628 \\ 6 & -0.651956 & 0.064145 & 0.427846 \\ 6 & -1.987632 & -0.108638 & -0.127263 \\ 8 & -2.359477 & 0.315608 & -1.218869 \\ 8 & -2.787875 & -0.850604 & 0.686130 \\ 6 & -4.084165 & -1.153608 & 0.152106 \\ 6 & 0.565556 & -1.467315 & -0.234117 \\ 6 & 1.865614 & -1.435003 & 0.321280 \\ 6 & 2.861948 & -0.587929 & -0.227539 \\ 8 & 2.600969 & 0.355523 & -1.017828 \\ 6 & -0.288072 & -2.686200 & 0.053745 \\ 6 & 4.320891 & -0.822792 & 0.157173 \\ 1 & 0.505829 & -1.106769 & -1.260400 \\ 1 & -1.282772 & -2.627747 & -0.393186 \\ 1 & 0.214528 & -3.567463 & -0.364508 \\ 1 & -0.406228 & -2.855046 & 1.129892 \\ 1 & 2.115853 & -2.121204 & 1.127419 \\ 1 & 4.445076 & -1.595551 & 0.922768 \\ 1 & 4.770791 & 0.112625 & 0.510894 \\ 1 & -0.573570 & 0.023481 & 1.511832 \\ 1 & -4.639668 & -0.236680 & -0.064105 \\ 1 & -4.589654 & -1.733241 & 0.925654 \\ 1 & -3.999967 & -1.738097 & -0.768571 \\ 1 & 4.881706 & -1.124183 & -0.735909 \\ 6 & 1.337217 & 1.940600 & 1.010574 \\ 6 & -1.129343 & 2.886378 & 0.106867 \\ 1 & -0.660278 & 3.843034 & -0.140977 \\ 1 & -1.427099 & 2.860809 & 1.157953 \\ 1 & -1.986093 & 2.706513 & -0.543116 \\ 1 & 2.131562 & 2.507789 & 0.526975 \\ 1 & 0.871619 & 2.499937 & 1.825375 \\ 1 & 1.737128 & 0.989620 & 1.362575\end{array}$

$\mathrm{E}(\mathrm{MeCN})=-1015.8799472$

NImag $=1(-310.1)$

49. I-3'c conformer1 (-1015.6793554)

$\begin{array}{rrrr}16 & -0.276996 & 1.456207 & -0.655156 \\ 6 & 1.167240 & 2.641587 & -0.810599 \\ 6 & -0.771649 & 1.815707 & 1.062821 \\ 6 & 0.644282 & -0.152465 & -0.601664 \\ 6 & -0.418755 & -1.190064 & -0.099458 \\ 6 & -1.763563 & -0.784253 & -0.628603 \\ 6 & -2.835389 & -0.551086 & 0.250985 \\ 8 & -2.711485 & -0.393754 & 1.501770 \\ 6 & -4.239628 & -0.415083 & -0.338835 \\ 6 & 0.012876 & -2.617320 & -0.477719\end{array}$

$\begin{array}{rrr}1.673258 & 2.435119 & -1.757843 \\ 1.856248 & 2.549479 & 0.029951 \\ 0.734193 & 3.644032 & -0.847202 \\ 0.121659 & 1.849891 & 1.685521 \\ -1.272416 & 2.786851 & 1.024252 \\ -1.478166 & 1.030814 & 1.397367 \\ -0.468837 & -1.104974 & 0.993045 \\ -1.965341 & -0.973719 & -1.682103 \\ -4.672000 & 0.549404 & -0.046039 \\ -4.884326 & -1.191710 & 0.090456 \\ -4.263810 & -0.502632 & -1.430494 \\ -0.707621 & -3.326526 & -0.060126 \\ 0.018497 & -2.749408 & -1.567284 \\ 1.009739 & -2.878196 & -0.101399 \\ 0.874361 & -0.340232 & -1.656217 \\ 1.927428 & -0.128614 & 0.205340 \\ 2.098826 & 0.457908 & 1.256155 \\ 2.855636 & -0.916762 & -0.370053 \\ 4.075072 & -1.090944 & 0.379605 \\ 4.686123 & -1.768319 & -0.216354 \\ 4.578868 & -0.130383 & 0.512852 \\ 3.860211 & -1.522729 & 1.359908\end{array}$

$\mathrm{E}(\mathrm{MeCN})=-1015.9019754$

$\mathrm{NImag}=0$

50. I-3' $c$ conformer2 (-1015.68029)

$\begin{array}{crrr}16 & 0.460036 & -1.392374 & -0.387632 \\ 6 & -1.001580 & -2.266797 & -1.182457 \\ 6 & 0.268477 & -2.196330 & 1.248687 \\ 6 & -0.363913 & 0.301664 & -0.375303 \\ 6 & 0.502949 & 1.420155 & 0.281799 \\ 6 & 1.883746 & 1.481036 & -0.306342 \\ 6 & 2.737798 & 0.464211 & 0.002966 \\ 8 & 2.309059 & -0.577538 & 0.668100 \\ 6 & 4.195900 & 0.462437 & -0.406486 \\ 6 & -0.237081 & 2.763219 & 0.158718 \\ 1 & -1.211742 & -1.784516 & -2.140291 \\ 1 & -1.897864 & -2.263730 & -0.556245 \\ 1 & -0.685605 & -3.297734 & -1.365109 \\ 1 & -0.519292 & -1.688204 & 1.806192 \\ 1 & -0.002073 & -3.239099 & 1.066258 \\ 1 & 1.235607 & -2.098330 & 1.733462 \\ 1 & 0.570592 & 1.147881 & 1.344742 \\ 1 & 2.185360 & 2.350521 & -0.882494 \\ 1 & 4.413285 & -0.406864 & -1.040444 \\ 1 & 4.830553 & 0.369604 & 0.483848 \\ 1 & 4.479260 & 1.373475 & -0.944433 \\ 1 & 0.380189 & 3.546743 & 0.609732\end{array}$




$\begin{array}{rrrr}1 & -0.404346 & 3.034458 & -0.891111 \\ 1 & -1.205791 & 2.765804 & 0.671721 \\ 1 & -0.447863 & 0.503221 & -1.446051 \\ 6 & -1.755467 & 0.231852 & 0.229454 \\ 8 & -2.013481 & -0.063346 & 1.379504 \\ 8 & -2.688475 & 0.606641 & -0.669831 \\ 6 & -4.036188 & 0.688661 & -0.167782 \\ 1 & -4.640313 & 1.021086 & -1.011735 \\ 1 & -4.372536 & -0.288826 & 0.187485 \\ 1 & -4.092657 & 1.405787 & 0.654884\end{array}$

$\mathrm{E}(\mathrm{MeCN})=-1015.8987749$ NImag $=0$

51. I-3'c conformer3 (-1015.6693755)

$\begin{array}{rrrr}16 & -0.437121 & 1.383504 & 0.150575 \\ 6 & -1.181669 & 2.234691 & -1.295537 \\ 6 & 0.913893 & 2.716260 & 0.377505 \\ 6 & 0.668637 & 0.020457 & -0.475271 \\ 6 & -0.297122 & -1.194908 & -0.332248 \\ 6 & -1.672077 & -0.606346 & -0.516650 \\ 6 & -2.656943 & -0.842273 & 0.504237 \\ 8 & -2.385568 & -1.255960 & 1.643279 \\ 6 & -4.114037 & -0.494676 & 0.179613 \\ 6 & 0.080625 & -2.341333 & -1.278204 \\ 1 & -2.242170 & 1.988264 & -1.290497 \\ 1 & -0.724718 & 1.865094 & -2.216459 \\ 1 & -1.017254 & 3.312265 & -1.207828 \\ 1 & 1.504861 & 2.879737 & -0.529649 \\ 1 & 0.394716 & 3.638051 & 0.652832 \\ 1 & 1.551088 & 2.394758 & 1.199989 \\ 1 & -0.245740 & -1.536758 & 0.709273 \\ 1 & -2.027614 & -0.508709 & -1.544555 \\ 1 & -4.509256 & 0.179993 & 0.947360 \\ 1 & -4.717909 & -1.409310 & 0.219220 \\ 1 & -4.245081 & -0.036378 & -0.807550 \\ 1 & -0.584699 & -3.192886 & -1.105750 \\ 1 & -0.028847 & -2.038957 & -2.328036 \\ 1 & 1.113630 & -2.680594 & -1.133320 \\ 1 & 0.875284 & 0.235230 & -1.529128 \\ 6 & 1.967033 & -0.128317 & 0.295953 \\ 8 & 2.115092 & 0.094269 & 1.478226 \\ 8 & 2.926333 & -0.638726 & -0.502765 \\ 6 & 4.165048 & -0.972266 & 0.155695 \\ 1 & 4.800755 & -1.397291 & -0.620796 \\ 1 & 4.621300 & -0.075282 & 0.581853 \\ 1 & 3.988392 & -1.697678 & 0.953377\end{array}$

$\mathrm{E}(\mathrm{MeCN})=-1015.8910967$ NImag $=0$
52. I-3'c conformer4 (-1015.6767493)

$\begin{array}{lrrr}16 & -0.324546 & 1.452251 & 0.355332 \\ 6 & -1.427884 & 1.927414 & -1.024900 \\ 6 & 1.098122 & 2.562881 & -0.161006 \\ 6 & 0.440588 & -0.150589 & -0.270345 \\ 6 & -0.347521 & -1.426584 & 0.174579 \\ 6 & -1.758316 & -1.476910 & -0.345643 \\ 6 & -2.670557 & -0.601712 & 0.174900 \\ 8 & -2.306839 & 0.385868 & 0.943098 \\ 6 & -4.153718 & -0.713432 & -0.129417 \\ 6 & 0.445657 & -2.677474 & -0.243539 \\ 1 & -1.781177 & 1.003416 & -1.487835 \\ 1 & -0.889409 & 2.550513 & -1.741908 \\ 1 & -2.270508 & 2.451849 & -0.577837 \\ 1 & 1.481167 & 2.331319 & -1.159428 \\ 1 & 0.713624 & 3.586472 & -0.136299 \\ 1 & 1.888591 & 2.461853 & 0.584467 \\ 1 & -0.364750 & -1.373357 & 1.273532 \\ 1 & -2.050035 & -2.296854 & -0.995173 \\ 1 & -4.521471 & 0.209760 & -0.596592 \\ 1 & -4.711231 & -0.833225 & 0.807707 \\ 1 & -4.385711 & -1.559510 & -0.785130 \\ 1 & -0.124005 & -3.565305 & 0.049069 \\ 1 & 0.590551 & -2.717078 & -1.330148 \\ 1 & 1.428473 & -2.740125 & 0.236437 \\ 1 & 0.453030 & -0.071225 & -1.360384 \\ 6 & 1.859176 & -0.188139 & 0.268611 \\ 8 & 2.160524 & -0.071321 & 1.437747 \\ 8 & 2.746193 & -0.417623 & -0.721780 \\ 6 & 4.112679 & -0.583856 & -0.297070 \\ 1 & 4.680131 & -0.766138 & -1.209520 \\ 1 & 4.468560 & 0.318319 & 0.207020 \\ 1 & 4.198528 & -1.431685 & 0.387165\end{array}$

$\mathrm{E}(\mathrm{MeCN})=-1015.896434$

NImag $=0$

53. TS-3't (-1015.65622)

$\begin{array}{lccc}16 & -1.498212 & -1.512555 & -0.204053 \\ 6 & -2.626477 & -1.216180 & -1.619407 \\ 6 & -2.648405 & -1.221489 & 1.181382 \\ 6 & -0.293762 & -0.182781 & -0.171280 \\ 6 & 0.932593 & -0.376049 & 1.276735 \\ 6 & 2.229310 & -0.020548 & 0.798872 \\ 6 & 2.778614 & -0.745303 & -0.278425 \\ 8 & 2.128034 & -1.642887 & -0.887359 \\ 6 & 4.183068 & -0.411299 & -0.771359 \\ 6 & 0.403442 & 0.397825 & 2.472227 \\ 1 & -2.029577 & -1.341139 & -2.525158\end{array}$




$\begin{array}{lrrr}1 & -3.419299 & -1.968781 & -1.592546 \\ 1 & -3.025729 & -0.203942 & -1.546072 \\ 1 & -3.470345 & -1.933929 & 1.076974 \\ 1 & -2.993504 & -0.188517 & 1.143351 \\ 1 & -2.103813 & -1.425852 & 2.103952 \\ 1 & 0.455959 & -0.511692 & -0.902524 \\ 1 & 0.787603 & -1.457712 & 1.353143 \\ 1 & -0.644376 & 0.180743 & 2.704474 \\ 1 & 1.001559 & 0.152044 & 3.357944 \\ 1 & 0.492442 & 1.476803 & 2.304889 \\ 1 & 2.688172 & 0.905955 & 1.133702 \\ 1 & 4.664244 & 0.379866 & -0.186405 \\ 1 & 4.807337 & -1.311840 & -0.726018 \\ 1 & 4.145024 & -0.104702 & -1.823975 \\ 6 & -0.869259 & 1.163163 & -0.295090 \\ 8 & -2.047417 & 1.480142 & -0.123612 \\ 8 & 0.093543 & 2.069716 & -0.568878 \\ 6 & -0.337073 & 3.438508 & -0.610268 \\ 1 & 0.564490 & 4.018209 & -0.810226 \\ 1 & -1.072818 & 3.588259 & -1.405441 \\ 1 & -0.781579 & 3.736254 & 0.343254\end{array}$

$\mathrm{E}(\mathrm{MeCN})=-1015.880081$

NImag $=1(-343.3)$

54. TS-3't conformer2 (-1015.6644002)

$\begin{array}{crrr}16 & 0.087622 & 1.551257 & -0.260628 \\ 6 & -0.651956 & 0.064145 & 0.427846 \\ 6 & -1.987632 & -0.108638 & -0.127263 \\ 8 & -2.359477 & 0.315608 & -1.218869 \\ 8 & -2.787875 & -0.850604 & 0.686130 \\ 6 & -4.084165 & -1.153608 & 0.152106 \\ 6 & 0.565556 & -1.467315 & -0.234117 \\ 6 & 1.865614 & -1.435003 & 0.321280 \\ 6 & 2.861948 & -0.587929 & -0.227539 \\ 8 & 2.600969 & 0.355523 & -1.017828 \\ 6 & -0.288072 & -2.686200 & 0.053745 \\ 6 & 4.320891 & -0.822792 & 0.157173 \\ 1 & 0.505829 & -1.106769 & -1.260400 \\ 1 & -1.282772 & -2.627747 & -0.393186 \\ 1 & 0.214528 & -3.567463 & -0.364508 \\ 1 & -0.406228 & -2.855046 & 1.129892 \\ 1 & 2.115853 & -2.121204 & 1.127419 \\ 1 & 4.445076 & -1.595551 & 0.922768 \\ 1 & 4.770791 & 0.112625 & 0.510894 \\ 1 & -0.573570 & 0.023481 & 1.511832 \\ 1 & -4.639668 & -0.236680 & -0.064105 \\ 1 & -4.589654 & -1.733241 & 0.925654 \\ 1 & -3.999967 & -1.738097 & -0.768571\end{array}$

$\begin{array}{rrr}4.881706 & -1.124183 & -0.735909 \\ 1.337217 & 1.940600 & 1.010574 \\ -1.129343 & 2.886378 & 0.106867 \\ -0.660278 & 3.843034 & -0.140977 \\ -1.427099 & 2.860809 & 1.157953 \\ -1.986093 & 2.706513 & -0.543116 \\ 2.131562 & 2.507789 & 0.526975 \\ 0.871619 & 2.499937 & 1.825375 \\ 1.737128 & 0.989620 & 1.362575\end{array}$

$\mathrm{E}(\mathrm{MeCN})=-1015.8846098$

NImag $=1(-322.0)$

55. I-3't (-1015.67304)

$\begin{array}{crrr}16 & -1.298232 & -1.699035 & 0.336554 \\ 6 & 0.078717 & -2.899291 & 0.521514 \\ 6 & -1.776107 & -2.069678 & -1.383841 \\ 6 & -0.290053 & -0.094407 & 0.089594 \\ 6 & 0.710400 & 0.183496 & 1.272742 \\ 6 & 1.930404 & 0.777410 & 0.641205 \\ 6 & 2.566162 & 0.083104 & -0.372137 \\ 8 & 2.164185 & -1.064404 & -0.814249 \\ 6 & 3.778331 & 0.692496 & -1.063103 \\ 6 & 0.107729 & 1.015142 & 2.409011 \\ 1 & 0.243937 & -3.052621 & 1.588805 \\ 1 & -0.245046 & -3.837944 & 0.064636 \\ 1 & 0.970587 & -2.468527 & 0.035367 \\ 1 & -2.196821 & -3.077410 & -1.411680 \\ 1 & -0.888897 & -2.002934 & -2.019750 \\ 1 & -2.526331 & -1.332724 & -1.668161 \\ 1 & 0.357027 & -0.392294 & -0.754850 \\ 1 & 0.951014 & -0.809561 & 1.683440 \\ 1 & -0.809698 & 0.567768 & 2.814084 \\ 1 & 0.833886 & 1.091800 & 3.225157 \\ 1 & -0.123105 & 2.032218 & 2.076671 \\ 1 & 2.229559 & 1.782113 & 0.921679 \\ 1 & 4.062098 & 1.664873 & -0.645608 \\ 1 & 4.633701 & 0.010183 & -0.980393 \\ 1 & 3.574612 & 0.812504 & -2.135105 \\ 6 & -1.317933 & 0.952797 & -0.239038 \\ 8 & -2.515206 & 0.723457 & -0.349956 \\ 8 & -0.777281 & 2.163143 & -0.416382 \\ 6 & -1.705578 & 3.219564 & -0.728729 \\ 1 & -1.092848 & 4.113976 & -0.837844 \\ 1 & -2.235905 & 2.998273 & -1.658253 \\ 1 & -2.432077 & 3.343499 & 0.078331\end{array}$

$\mathrm{E}(\mathrm{MeCN})=-1015.8951576$

NImag $=0$ 


\begin{tabular}{|c|c|c|c|c|c|c|c|}
\hline \multicolumn{4}{|c|}{ 56. TS-3'R (-1015.66564) } & \multirow{3}{*}{$\begin{array}{l}6 \\
8 \\
8\end{array}$} & \multirow{2}{*}{$\begin{array}{l}0.228992 \\
2.122877\end{array}$} & \multirow{2}{*}{$\begin{array}{r}0.917644 \\
-1.346920\end{array}$} & \multirow{2}{*}{$\begin{array}{r}2.445512 \\
-0.714065\end{array}$} \\
\hline & & & & & & & \\
\hline 6 & 1.345942 & 2.224699 & .276210 & & -2.352316 & 0.999529 & -0.506266 \\
\hline 1 & 1.314647 & 2.136586 & 1.364898 & 1 & -2.626452 & -1.074341 & -1.753085 \\
\hline 1 & 2.122654 & 1.538875 & -.177921 & 1 & -2.485932 & -2.844509 & -1.489080 \\
\hline 16 & -.254879 & 1.754119 & -.429876 & 1 & -1.051061 & -1.904403 & -2.021470 \\
\hline 6 & -1.424283 & 2.749925 & .554413 & 1 & -0.754178 & -3.817040 & 0.144168 \\
\hline 1 & -1.297410 & 2.541500 & 1.619787 & 1 & 0.612589 & -2.610473 & 0.086641 \\
\hline 1 & -2.432206 & 2.503017 & .219413 & 1 & -0.198058 & -3.030446 & 1.643571 \\
\hline 6 & -.517667 & .034326 & .203979 & 1 & 0.302464 & -0.474125 & -0.751373 \\
\hline 1 & -.292700 & .036962 & 1.272035 & 1 & -0.716298 & 0.530049 & 2.848659 \\
\hline 6 & .538413 & -.961532 & -.536103 & 1 & 0.971767 & 0.896458 & 3.249800 \\
\hline 6 & -.180788 & -2.228455 & -1.031299 & 1 & 0.076500 & 1.964302 & 2.164056 \\
\hline 1 & .534816 & -2.815729 & -1.613193 & 1 & 2.284476 & 1.627612 & 0.775943 \\
\hline 1 & -.520083 & -2.849817 & -.195410 & 1 & 4.630525 & -0.389877 & -0.979237 \\
\hline 1 & -1.043207 & -2.010208 & -1.675645 & 1 & 3.600059 & 0.366203 & -2.189857 \\
\hline 6 & 1.722573 & -1.264267 & .310627 & 1 & 4.127124 & 1.306568 & -0.768410 \\
\hline 1 & 1.640143 & -2.091100 & 1.013488 & 1 & 0.925912 & -0.935155 & 1.622161 \\
\hline 6 & 2.946806 & -.660031 & .076921 & 1 & -0.632596 & 4.274067 & -0.626320 \\
\hline 6 & 4.168663 & -1.156941 & .846340 & 1 & -1.769312 & 3.306996 & -1.624830 \\
\hline 1 & 3.944812 & -1.985069 & 1.528381 & 1 & -2.111651 & 3.562588 & 0.104416 \\
\hline 1 & 4.936339 & -1.483963 & .134052 & & & & \\
\hline 1 & 4.608422 & -.331538 & 1.421520 & \multicolumn{4}{|c|}{$\mathrm{E}(\mathrm{MeCN})=-1015.8938961$} \\
\hline 8 & 3.144106 & .312085 & -.741246 & \multirow{2}{*}{\multicolumn{4}{|c|}{ NImag $=1(-84.1)$}} \\
\hline 6 & -1.983949 & -.267848 & -.028513 & \multirow{3}{*}{\multicolumn{4}{|c|}{ 58. PC-3' (-1015.75355) }} \\
\hline 8 & -2.682378 & .247549 & -.883608 & & & & \\
\hline 1 & -1.207794 & 3.801049 & .348935 & & & & \\
\hline 1 & 1.514822 & 3.261432 & -.028379 & 6 & 2.532029 & -1.459228 & -1.197125 \\
\hline 8 & -2.408155 & -1.212385 & .824900 & 16 & 3.068240 & -1.359589 & 0.547506 \\
\hline 1 & .897610 & -.406391 & -1.414040 & 6 & -0.674087 & -0.062275 & 0.290686 \\
\hline 6 & -3.754732 & -1.682287 & .616404 & 6 & -1.465991 & -1.230777 & -0.183413 \\
\hline 1 & -3.927985 & -2.417890 & 1.401249 & 8 & -2.794338 & -0.979374 & -0.303807 \\
\hline 1 & -4.464069 & -.854920 & .695290 & 6 & -3.585685 & -2.087393 & -0.765392 \\
\hline 1 & -3.845214 & -2.142234 & -.370689 & 6 & 4.064144 & 0.171521 & 0.477821 \\
\hline & & & & 6 & -1.087734 & 0.901291 & 1.377426 \\
\hline \multicolumn{4}{|c|}{$\mathrm{E}(\mathrm{MeCN})=-1015.8939684$} & 6 & -1.095561 & 1.370140 & -0.064614 \\
\hline \multicolumn{4}{|c|}{ NImag = $1(-20.1)$} & 6 & -0.027493 & 2.308368 & -0.529113 \\
\hline \multirow{3}{*}{\multicolumn{4}{|c|}{ 57. TS-3'E (-1015.67288) }} & 6 & -0.456606 & 3.396374 & -1.495095 \\
\hline & & & & 6 & -2.360222 & 0.801277 & 2.189940 \\
\hline & & & & 8 & 1.127269 & 2.208459 & -0.143846 \\
\hline 6 & -1.971469 & -1.882563 & -1.430377 & 8 & -0.974364 & -2.306839 & -0.456808 \\
\hline 16 & -1.551472 & -1.555968 & 0.313607 & 1 & 1.734978 & -2.205564 & -1.246134 \\
\hline 6 & -0.267336 & -0.050681 & 0.086837 & 1 & 3.355960 & -1.754004 & -1.854489 \\
\hline 6 & -1.157339 & 1.111894 & -0.269281 & 1 & 2.128325 & -0.496201 & -1.525975 \\
\hline 8 & -0.509178 & 2.282104 & -0.309494 & 1 & 4.896577 & 0.072583 & -0.226236 \\
\hline 6 & -1.319469 & 3.428169 & -0.636415 & 1 & 3.436493 & 1.024699 & 0.205590 \\
\hline 6 & -0.332717 & -2.902185 & 0.568445 & 1 & 4.471544 & 0.333518 & 1.479653 \\
\hline 6 & 0.737405 & 0.087063 & 1.264992 & 1 & 0.393077 & -0.254232 & 0.248876 \\
\hline 6 & 1.965529 & 0.607815 & 0.588871 & 1 & -2.226880 & 0.098617 & 3.021124 \\
\hline 6 & 2.570980 & -0.191380 & -0.375469 & 1 & -2.611259 & 1.778881 & 2.618075 \\
\hline 6 & 3.803192 & 0.318109 & -1.112261 & 1 & -3.204650 & 0.459078 & 1.588647 \\
\hline
\end{tabular}




$\begin{array}{rrrr}1 & -2.072994 & 1.472325 & -0.527278 \\ 1 & 0.394854 & 4.033154 & -1.742400 \\ 1 & -0.862351 & 2.950431 & -2.412299 \\ 1 & -1.258153 & 4.003359 & -1.055359 \\ 1 & -0.222097 & 1.260147 & 1.931865 \\ 1 & -4.610931 & -1.717976 & -0.803324 \\ 1 & -3.254607 & -2.408247 & -1.756415 \\ 1 & -3.505032 & -2.930896 & -0.075052\end{array}$

$\mathrm{E}(\mathrm{MeCN})=-1015.9590585$

NImag $=0$

59. TS-4c (-1132.1769664)

\begin{tabular}{cccc}
6 & 1.216061 & -2.542494 & -1.944887 \\
1 & 0.294223 & -3.023596 & -1.615045 \\
1 & 1.036616 & -1.898423 & -2.808186 \\
16 & 1.834570 & -1.467083 & -0.602654 \\
6 & 2.005515 & -2.700286 & 0.734344 \\
1 & 1.019975 & -3.109672 & 0.951817 \\
1 & 2.426538 & -2.185626 & 1.597338 \\
6 & 0.452925 & -0.411486 & -0.186837 \\
1 & 0.523075 & 0.435993 & -0.877887 \\
6 & 0.992685 & 0.745648 & 1.290807 \\
6 & 0.894367 & 0.001268 & 2.608524 \\
1 & 1.825208 & -0.519193 & 2.862049 \\
1 & 0.708765 & 0.726011 & 3.411134 \\
1 & 0.073498 & -0.723317 & 2.622547 \\
1 & 0.118514 & 1.369865 & 1.108045 \\
6 & 2.219927 & 1.370306 & 0.931675 \\
1 & 3.139256 & 1.110576 & 1.451343 \\
6 & 2.245197 & 2.222875 & -0.198427 \\
6 & 3.534916 & 2.955589 & -0.552754 \\
1 & 4.361675 & 2.720013 & 0.125649 \\
1 & 3.832140 & 2.711646 & -1.579691 \\
1 & 3.355255 & 4.036960 & -0.524045 \\
8 & 1.242803 & 2.390314 & -0.946969 \\
6 & -0.843747 & -1.064037 & -0.015740 \\
8 & -0.959713 & -2.281710 & 0.235542 \\
1 & 2.705372 & -3.461683 & 0.380868 \\
1 & 1.993682 & -3.272838 & -2.183497 \\
6 & -2.060750 & -0.192404 & -0.080080 \\
6 & -3.296030 & -0.786181 & 0.233331 \\
6 & -4.471766 & -0.045592 & 0.181195 \\
6 & -4.432302 & 1.303313 & -0.186466 \\
6 & -3.211653 & 1.902346 & -0.500055 \\
6 & -2.027132 & 1.165253 & -0.450198 \\
1 & -3.302469 & -1.833970 & 0.514691 \\
1 & -5.420365 & -0.515996 & 0.426971 \\
1 & -5.350694 & 1.883369 & -0.228686 \\
1 & -3.175727 & 2.949542 & -0.787635 \\
1 & -1.091665 & 1.660139 & -0.700488 \\
& & & \\
\hline
\end{tabular}

$\mathrm{E}(\mathrm{MeCN})=-1132.4161151$

NImag $=1(-350.5)$
60. TS-4 $c$ conformer2 (-1132.1783136)

$$
\begin{array}{ccc}
3.019419 & -1.260624 & -.879085 \\
2.757150 & -.853017 & -1.857979 \\
3.373919 & -. .55311 & -.232659 \\
1.549415 & -1.977734 & -.078742 \\
1.006658 & -3.124506 & -1.403722 \\
.888161 & -2.575314 & -2.340361 \\
.056732 & -3.538881 & -1.068435 \\
.419164 & -.586731 & -.096378 \\
.602724 & .108738 & -.915386 \\
.959550 & .690877 & 1.344869 \\
.914174 & -.069967 & 2.654318 \\
1.831423 & -.647035 & 2.822600 \\
.823665 & .643879 & 3.482830 \\
.063603 & -.756142 & 2.708712 \\
.052201 & 1.256726 & 1.140892 \\
2.151943 & 1.370154 & .978854 \\
3.067101 & 1.196250 & 1.542029 \\
2.161005 & 2.208473 & -.164896 \\
3.408543 & 3.035552 & -.467727 \\
4.223560 & 2.863145 & .243426 \\
3.763321 & 2.819713 & -1.482787 \\
3.147008 & 4.100194 & -.445875 \\
1.191399 & 2.287289 & -.964078 \\
-.920109 & -1.092803 & .162929 \\
-1.075292 & -2.252991 & .603888 \\
1.763570 & -3.907520 & -1.501271 \\
3.769779 & -2.049448 & -.970133 \\
-2.089542 & -.189422 & -.045427 \\
-3.362222 & -.688966 & .281370 \\
-4.496221 & .094515 & .098011 \\
-4.374273 & 1.390210 & -.415167 \\
-3.11408 & 1.894949 & -.739920 \\
-1.970686 & 1.114925 & -.559766 \\
-3.431095 & -1.696586 & .677971 \\
-5.475920 & -.300436 & .354146 \\
-5.260242 & 2.003333 & -.559987 \\
-3.015491 & 2.902124 & -1.135470 \\
-.998632 & 1.535127 & -.810444 \\
& &
\end{array}
$$

$\mathrm{E}(\mathrm{MeCN})=-1132.4207129$

NImag $=1$

61. I-4 $c$ conformer1 (-1132.1904898)

$\begin{array}{cccc}6 & -2.621422 & 0.751739 & -1.701164 \\ 1 & -2.392167 & 1.335057 & -2.595606 \\ 1 & -2.260701 & -0.281975 & -1.795080 \\ 16 & -1.804169 & 1.516805 & -0.246885 \\ 6 & -1.044310 & 2.963828 & -1.113755 \\ 1 & -0.397690 & 2.641588 & -1.932779 \\ 1 & -0.467879 & 3.525496 & -0.382550 \\ 6 & -0.364286 & 0.382818 & 0.115063\end{array}$




\begin{tabular}{|c|c|c|c|c|c|c|c|}
\hline 1 & -0.431230 & -0.363599 & -0.685578 & 6 & -2.211045 & -1.094841 & 1.000304 \\
\hline 6 & -0.813209 & -0.404717 & 1.395230 & 1 & -2.812153 & -1.075727 & 1.905701 \\
\hline 6 & -0.744972 & 0.425880 & 2.680559 & 6 & -2.686779 & -1.711228 & -0.166045 \\
\hline 1 & -1.437054 & 1.276267 & 2.649551 & 6 & -4.133151 & -2.195750 & -0.213751 \\
\hline 1 & -1.038446 & -0.200741 & 3.529912 & 1 & -4.670713 & -2.045545 & 0.728960 \\
\hline 1 & 0.259993 & 0.814594 & 2.875200 & 1 & -4.670748 & -1.677156 & -1.017444 \\
\hline 1 & -0.074090 & -1.218642 & 1.466866 & 1 & -4.149134 & -3.263827 & -0.462092 \\
\hline 6 & -2.185407 & -0.956347 & 1.118429 & 8 & -1.992117 & -1.831799 & -1.223880 \\
\hline 1 & -2.935946 & -0.862548 & 1.898165 & 6 & 0.930138 & 0.914731 & 0.037316 \\
\hline 6 & -2.392765 & -1.819242 & 0.044682 & 8 & 0.959588 & 2.119604 & 0.294484 \\
\hline 6 & -3.745123 & -2.510998 & -0.103006 & 1 & -2.789612 & 3.151365 & 0.332016 \\
\hline 1 & -4.447216 & -2.259628 & 0.699310 & 1 & -2.004894 & 2.850132 & -2.216033 \\
\hline 1 & -4.199569 & -2.253621 & -1.068623 & 6 & 2.198590 & 0.133098 & -0.087211 \\
\hline 1 & -3.594868 & -3.597449 & -0.110696 & 6 & 3.396913 & 0.778397 & 0.266827 \\
\hline 8 & -1.531083 & -2.030640 & -0.882259 & 6 & 4.613728 & 0.114603 & 0.161895 \\
\hline 6 & 1.001719 & 1.027098 & 0.220906 & 6 & 4.652460 & -1.204421 & -0.302388 \\
\hline 8 & 1.151949 & 2.197856 & 0.559902 & 6 & 3.469896 & -1.853140 & -0.660609 \\
\hline 1 & -1.880550 & 3.553999 & -1.497068 & 6 & 2.246372 & -1.191447 & -0.556335 \\
\hline 1 & -3.693152 & 0.757412 & -1.498470 & 1 & 3.343896 & 1.802229 & 0.621883 \\
\hline 6 & 2.176817 & 0.135332 & -0.029283 & 1 & 5.533574 & 0.621184 & 0.441033 \\
\hline 6 & 3.457073 & 0.682844 & 0.167243 & 1 & 5.603625 & -1.723327 & -0.386458 \\
\hline 6 & 4.594224 & -0.083385 & -0.060447 & 1 & 3.497368 & -2.875507 & -1.026497 \\
\hline 6 & 4.467054 & -1.410106 & -0.485470 & 1 & 1.340296 & -1.712449 & -0.849319 \\
\hline 6 & 3.200769 & -1.963532 & -0.680197 & & & & \\
\hline 6 & 2.054537 & -1.199966 & -0.457026 & \multicolumn{4}{|c|}{$\mathrm{E}(\mathrm{MeCN})=-1132.4269517$} \\
\hline 1 & 3.530613 & 1.713359 & 0.498855 & \multicolumn{4}{|c|}{ NImag $=0$} \\
\hline 1 & 5.579599 & 0.347951 & 0.093434 & & & & \\
\hline 1 & 5.355300 & -2.010628 & -0.663717 & \multirow{2}{*}{\multicolumn{4}{|c|}{ 63. I-4c conformer3 (-1132.1835951) }} \\
\hline 1 & 3.100329 & -2.993855 & -1.009205 & & & & \\
\hline \multirow[t]{2}{*}{1} & 1.080292 & -1.657122 & -0.613490 & 6 & -0.473238 & 3.091042 & -0.137572 \\
\hline & & & & 16 & -1.449238 & 1.516921 & 0.200050 \\
\hline \multicolumn{4}{|c|}{$\mathrm{E}(\mathrm{MeCN})=-1132.4282528$} & 6 & -0.173589 & 0.299700 & -0.372656 \\
\hline \multirow{2}{*}{\multicolumn{4}{|c|}{ NImag $=0$}} & 6 & 0.980291 & 0.391990 & 0.619717 \\
\hline & & & & 6 & -2.558322 & 1.613410 & -1.243337 \\
\hline \multirow{2}{*}{\multicolumn{4}{|c|}{ 62. I-4 $c$ conformer2 (-1132.1868746) }} & 6 & -0.962770 & -1.080162 & -0.396832 \\
\hline & & & & 6 & -2.116335 & -1.011729 & 0.559306 \\
\hline 6 & -1.183354 & 2.221462 & -1.863145 & 6 & -3.432164 & -1.171955 & 0.084425 \\
\hline 1 & -0.352172 & 2.823261 & -1.494267 & 6 & -4.548808 & -1.387582 & 1.105556 \\
\hline 1 & -0.862929 & 1.565783 & -2.676696 & 6 & -0.045673 & -2.295817 & -0.181543 \\
\hline 16 & -1.876796 & 1.126911 & -0.543135 & 8 & -3.763302 & -1.100738 & -1.136349 \\
\hline 6 & -2.109315 & 2.402221 & 0.747074 & 8 & 0.758420 & 0.782380 & 1.761885 \\
\hline 1 & -1.148305 & 2.841951 & 1.009494 & 1 & 0.302961 & 3.167154 & 0.625032 \\
\hline 1 & -2.578994 & 1.905009 & 1.595223 & 1 & -1.176178 & 3.920318 & -0.027318 \\
\hline 6 & -0.357974 & 0.144480 & -0.109699 & 1 & -0.038112 & 3.102279 & -1.140876 \\
\hline 1 & -0.380280 & -0.536679 & -0.968276 & 1 & -3.311762 & 2.366331 & -0.996725 \\
\hline 6 & -0.750028 & -0.774867 & 1.115246 & 1 & -1.991595 & 1.928075 & -2.123719 \\
\hline 6 & -0.357241 & -0.200729 & 2.482058 & 1 & -3.043870 & 0.631090 & -1.398008 \\
\hline 1 & -0.865593 & 0.743692 & 2.705210 & 1 & 0.143894 & 0.605789 & -1.374622 \\
\hline 1 & -0.642758 & -0.916245 & 3.260916 & 1 & 0.779642 & -2.351435 & -0.901938 \\
\hline 1 & 0.721541 & -0.029347 & 2.566102 & 1 & 0.384038 & -2.304114 & 0.827331 \\
\hline 1 & -0.138312 & -1.677217 & 0.946635 & 1 & -0.649297 & -3.201941 & -0.289089 \\
\hline
\end{tabular}




$\begin{array}{lrrr}1 & -1.401253 & -1.149735 & -1.398990 \\ 1 & -1.907102 & -1.160015 & 1.616680 \\ 1 & -5.333893 & -0.635837 & 0.960013 \\ 1 & -5.013247 & -2.365441 & 0.927655 \\ 1 & -4.200730 & -1.348392 & 2.143199 \\ 6 & 2.360787 & 0.006029 & 0.197928 \\ 6 & 2.713996 & -0.302775 & -1.126215 \\ 6 & 4.031512 & -0.624572 & -1.448036 \\ 6 & 5.010919 & -0.641929 & -0.453148 \\ 6 & 4.670330 & -0.334383 & 0.867472 \\ 6 & 3.356775 & -0.011044 & 1.190045 \\ 1 & 1.968017 & -0.299139 & -1.914245 \\ 1 & 4.292725 & -0.862094 & -2.475287 \\ 1 & 6.037195 & -0.893817 & -0.706152 \\ 1 & 5.430603 & -0.347897 & 1.643368\end{array}$

$\mathrm{E}(\mathrm{MeCN})=-1132.4263796$

NImag $=0$

64. I-4c conformer4 (-1132.1823627)

$\begin{array}{crrrr}6 & 0.483861 & 2.825029 & -0.839671 & 6 \\ 16 & 1.651174 & 1.420959 & -0.424890 & 8 \\ 6 & 0.347225 & 0.392257 & 0.457333 & 6 \\ 6 & -0.649621 & -0.034673 & -0.608966 & 1 \\ 6 & 2.487683 & 2.313314 & 0.932498 & 1 \\ 6 & 0.966946 & -0.840971 & 1.241215 & 1 \\ 6 & 1.738713 & -1.790658 & 0.387464 & 1 \\ 6 & 2.935364 & -1.356562 & -0.112808 & 1 \\ 6 & 3.851649 & -2.259601 & -0.913936 & 1 \\ 6 & -0.126501 & -1.535497 & 2.068900 & 1 \\ 8 & 3.334006 & -0.130670 & 0.075066 & 1 \\ 8 & -0.239768 & -0.375470 & -1.711901 & 1 \\ 1 & -0.185172 & 2.476180 & -1.628479 & 1 \\ 1 & 1.090638 & 3.648269 & -1.227601 & 1 \\ 1 & -0.094179 & 3.164709 & 0.025802 & 1 \\ 1 & 2.971952 & 3.188445 & 0.491698 & 1 \\ 1 & 1.762319 & 2.632334 & 1.686263 & 1 \\ 1 & 3.223758 & 1.618698 & 1.330154 & 1 \\ 1 & -0.093592 & 1.084401 & 1.182604 & 6 \\ 1 & -0.687149 & -0.838640 & 2.704054 & 8 \\ 1 & -0.844919 & -2.067357 & 1.435997 & 6 \\ 1 & 0.350452 & -2.276537 & 2.718608 & 6 \\ 1 & 1.663182 & -0.357274 & 1.942871 & 6 \\ 1 & 1.347617 & -2.784264 & 0.193141 & 6 \\ 1 & 4.007152 & -1.843599 & -1.917282 & 6 \\ 1 & 4.837565 & -2.308330 & -0.434427 & 6 \\ 1 & 3.456890 & -3.276679 & -1.009856 & 1 \\ 6 & -2.112600 & -0.093276 & -0.291713 & 1 \\ 6 & -2.708178 & 0.533593 & 0.815293 & 1 \\ 6 & -4.086379 & 0.460342 & 1.013646 & 1\end{array}$

$$
\begin{array}{rrr}
-4.887558 & -0.245856 & 0.114951 \\
-4.305744 & -0.874288 & -0.990177 \\
-2.932724 & -0.792695 & -1.195388 \\
-2.107857 & 1.090439 & 1.527267 \\
-4.533889 & 0.954782 & 1.871349 \\
-5.960929 & -0.304981 & 0.273562 \\
-4.925464 & -1.425348 & -1.692164 \\
-2.463880 & -1.264221 & -2.052482
\end{array}
$$

$\mathrm{E}(\mathrm{MeCN})=-1132.4239486$

NImag $=0$

65. TS-4t (-1132.1622755)

$\begin{array}{rrr}3.439382 & -0.153995 & 1.170306 \\ 2.863384 & -0.615233 & -0.501885 \\ 2.927810 & -2.450099 & -0.358088 \\ 1.144538 & -0.037274 & -0.570394 \\ 1.081779 & 1.817200 & -0.248544 \\ 2.113229 & 2.433954 & -1.186572 \\ -0.246659 & 2.322754 & -0.367457 \\ -1.136353 & 2.128242 & 0.717451 \\ -0.824329 & 1.431507 & 1.713102 \\ -2.524571 & 2.760643 & 0.668649 \\ 3.484313 & 0.934539 & 1.214796 \\ 4.440970 & -0.572453 & 1.293973 \\ 2.728173 & -0.549091 & 1.894653 \\ 3.968645 & -2.726554 & -0.168854 \\ 2.265565 & -2.765116 & 0.447349 \\ 2.606599 & -2.853861 & -1.320317 \\ 0.900119 & -0.072027 & -1.631152 \\ 1.391995 & 1.773182 & 0.797017 \\ 3.118413 & 2.002924 & -1.097594 \\ 2.196492 & 3.504076 & -0.966426 \\ 1.805323 & 2.340412 & -2.235114 \\ -0.567739 & 2.809585 & -1.285380 \\ -2.667635 & 3.414818 & -0.198576 \\ -2.691052 & 3.340662 & 1.583638 \\ -3.285594 & 1.971420 & 0.645530 \\ 0.242847 & -0.847622 & 0.318409 \\ 0.701334 & -1.444803 & 1.303316 \\ -1.188474 & -1.003850 & -0.066348 \\ -2.009294 & -1.753030 & 0.795677 \\ -3.346682 & -1.968646 & 0.491172 \\ -3.893757 & -1.437021 & -0.683283 \\ -3.091229 & -0.691965 & -1.545425 \\ -1.744236 & -0.477282 & -1.243377 \\ -1.569683 & -2.142549 & 1.707052 \\ -3.970564 & -2.544347 & 1.169796 \\ -4.940661 & -1.605637 & -0.922378 \\ -3.510078 & -0.275438 & -2.457662\end{array}$




\begin{tabular}{|c|c|c|c|c|c|c|c|}
\hline 1 & -1.143765 & 0.113497 & -1.924598 & $\begin{array}{l}1 \\
1\end{array}$ & $\begin{array}{l}-3.991527 \\
-2.590852\end{array}$ & $\begin{array}{r}-0.178498 \\
0.896294\end{array}$ & $\begin{array}{l}-1.484710 \\
-0.992437\end{array}$ \\
\hline \multicolumn{4}{|c|}{$\mathrm{E}(\mathrm{MeCN})=-1132.4049269$} & 16 & -2.117979 & -1.508104 & -0.832470 \\
\hline \multicolumn{4}{|c|}{ NImag $=1(-371)$} & 6 & -3.353571 & -2.018636 & 0.408982 \\
\hline & & & & 1 & -3.610214 & -1.184661 & 1.064870 \\
\hline \multirow{2}{*}{\multicolumn{4}{|c|}{ 66. TS-4 $t$ conformer2 (-1132.1557383) }} & 1 & -2.935200 & -2.843760 & 0.989491 \\
\hline & & & & 6 & -0.733802 & -0.912594 & 0.312398 \\
\hline 6 & -2.873756 & -2.439193 & -.177220 & 1 & -0.410367 & -1.859093 & 0.773623 \\
\hline 16 & -2.842167 & -.594790 & -.241890 & 6 & -1.118317 & 0.180141 & 1.347816 \\
\hline 6 & -3.817824 & -.283714 & 1.270601 & 6 & -1.038670 & -0.334059 & 2.793188 \\
\hline 6 & -1.198968 & .033178 & .248794 & 1 & -0.013343 & -0.610986 & 3.067913 \\
\hline 6 & -.990643 & 1.899324 & -.199953 & 1 & -1.355834 & 0.456814 & 3.480004 \\
\hline 6 & -2.245123 & 2.599600 & .298719 & 1 & -1.678148 & -1.211086 & 2.965605 \\
\hline 6 & .250590 & 2.388082 & .283499 & 1 & -2.146133 & 0.511986 & 1.149041 \\
\hline 6 & 1.413513 & 2.192331 & -.512400 & 6 & -0.238191 & 1.367211 & 1.054678 \\
\hline 8 & 1.399530 & 1.513587 & -1.562807 & 1 & 0.715208 & 1.457850 & 1.563455 \\
\hline 6 & 2.730050 & 2.824837 & -.064325 & 6 & -0.768622 & 2.430223 & 0.314022 \\
\hline 1 & -2.234930 & -2.778055 & -.990535 & 6 & 0.095982 & 3.661969 & 0.071389 \\
\hline 1 & -3.909614 & -2.755451 & -.327057 & 1 & 1.067045 & 3.611251 & 0.575465 \\
\hline 1 & -2.494221 & -2.782868 & .787649 & 1 & 0.263779 & 3.784061 & -1.005711 \\
\hline 1 & -4.827806 & -.663409 & 1.100394 & 1 & -0.438019 & 4.556331 & 0.414048 \\
\hline 1 & -3.363110 & -.789763 & 2.126100 & 8 & -1.933261 & 2.424559 & -0.194491 \\
\hline 1 & -3.853674 & .790517 & 1.444041 & 6 & 0.324473 & -0.380293 & -0.681732 \\
\hline 1 & -1.119091 & -.004024 & 1.334696 & 8 & -0.053555 & -0.143723 & -1.835515 \\
\hline 1 & -.980330 & 1.739370 & -1.279140 & 1 & -4.238734 & -2.365178 & -0.128947 \\
\hline 1 & -3.170930 & 2.182757 & -.118065 & 1 & -2.573315 & 0.029511 & -2.572318 \\
\hline 1 & -2.205326 & 3.649038 & -.014389 & 6 & 1.775097 & -0.458276 & -0.348059 \\
\hline 1 & -2.312724 & 2.595292 & 1.394320 & 6 & 2.688646 & -0.021623 & -1.323041 \\
\hline 1 & .304129 & 2.902606 & 1.240566 & 6 & 4.058873 & -0.109438 & -1.098820 \\
\hline 1 & 2.622555 & 3.465622 & .818013 & 6 & 4.543778 & -0.650170 & 0.095580 \\
\hline 1 & 3.144448 & 3.418350 & -.887203 & 6 & 3.645006 & -1.099994 & 1.065255 \\
\hline 1 & 3.456524 & 2.034155 & .158442 & 6 & 2.272046 & -1.003326 & 0.847278 \\
\hline 6 & -.229455 & -.772578 & -.538854 & 1 & 2.296503 & 0.383415 & -2.249677 \\
\hline 8 & -.624154 & -1.288820 & -1.599788 & 1 & 4.752725 & 0.239893 & -1.858921 \\
\hline 6 & 1.139935 & -1.026522 & -.016990 & 1 & 5.614128 & -0.721047 & 0.269260 \\
\hline 6 & 2.007288 & -1.792512 & -.815187 & 1 & 4.013495 & -1.524723 & 1.995262 \\
\hline 6 & 3.282238 & -2.112131 & -.36 & 1 & 1.591593 & -1.345511 & 1.620754 \\
\hline 6 & 3.716153 & -1.671601 & .888837 & \multirow{2}{*}{\multicolumn{4}{|c|}{$\mathrm{E}(\mathrm{MeCN})=-1132.419925$}} \\
\hline 6 & 2.864140 & -.911427 & 1.689322 & & & & \\
\hline 6 & 1.579506 & -.593779 & 1.244156 & \multicolumn{4}{|c|}{ NImag $=0$} \\
\hline 1 & 1.656467 & -2.111974 & -1.790279 & \multirow{3}{*}{\multicolumn{4}{|c|}{ 68. I-4 $t$ conformer2 (-1132.1766205) }} \\
\hline 1 & 3.946093 & -2.698641 & -.996904 & & & & \\
\hline 1 & 4.713830 & -1.922436 & 1.239987 & & & & \\
\hline 1 & 3.196348 & -.565503 & 2.664652 & 16 & -2.322213 & -1.582714 & 0.201096 \\
\hline \multirow[t]{2}{*}{1} & \multirow[t]{2}{*}{.937162} & .009533 & 1.875301 & 6 & -3.100350 & -0.541427 & -1.087035 \\
\hline & & & & 6 & -1.700360 & -3.017222 & -0.738117 \\
\hline \multicolumn{4}{|c|}{$\mathrm{E}(\mathrm{MeCN})=-1132.4031308$} & 6 & -0.780808 & -0.674021 & 0.749507 \\
\hline \multirow{2}{*}{\multicolumn{4}{|c|}{ NImag = $1(-376.3)$}} & 6 & -1.163021 & 0.658042 & 1.451157 \\
\hline & & & & 6 & -0.997809 & 0.589938 & 2.974043 \\
\hline \multirow{2}{*}{\multicolumn{4}{|c|}{ 67. I-4t (-1132.176878) }} & 6 & -0.319465 & 1.700817 & 0.756988 \\
\hline & & & & 6 & -0.890719 & 2.439607 & -0.290070 \\
\hline 6 & -2.911161 & -0.017781 & -1.538350 & 8 & -2.047526 & 2.215959 & -0.756208 \\
\hline
\end{tabular}




\begin{tabular}{|c|c|c|c|}
\hline 6 & -0.066062 & 3.537121 & -0.951469 \\
\hline 1 & -4.178514 & -0.682707 & -0.982813 \\
\hline 1 & -2.733391 & -0.859311 & -2.061195 \\
\hline 1 & -2.792916 & 0.512384 & -0.916146 \\
\hline 1 & -2.565602 & -3.576895 & -1.101083 \\
\hline 1 & -1.081420 & -2.654484 & -1.559039 \\
\hline 1 & -1.121129 & -3.641639 & -0.053899 \\
\hline 1 & -0.333411 & -1.402163 & 1.441819 \\
\hline 1 & -1.607244 & -0.206391 & 3.421553 \\
\hline 1 & 0.047296 & 0.415829 & 3.259399 \\
\hline 1 & -1.303851 & 1.541207 & 3.420894 \\
\hline 1 & -2.211406 & 0.898961 & 1.226229 \\
\hline 1 & 0.623468 & 1.999331 & 1.202935 \\
\hline 1 & 0.131654 & 3.261104 & -1.994682 \\
\hline 1 & -0.644479 & 4.468124 & -0.970482 \\
\hline 1 & 0.889794 & 3.719198 & -0.448992 \\
\hline 6 & 0.170532 & -0.368815 & -0.446321 \\
\hline 8 & -0.276929 & -0.411094 & -1.598607 \\
\hline 6 & 1.649532 & -0.393573 & -0.204201 \\
\hline 6 & 2.483087 & -0.261028 & -1.328393 \\
\hline 6 & 3.867547 & -0.314591 & -1.198137 \\
\hline 6 & 4.449892 & -0.517606 & 0.055996 \\
\hline 6 & 3.632286 & -0.660779 & 1.178904 \\
\hline 6 & 2.245542 & -0.596549 & 1.051810 \\
\hline 1 & 2.015304 & -0.116400 & -2.296248 \\
\hline 1 & 4.496030 & -0.202508 & -2.077955 \\
\hline 1 & 5.530878 & -0.562350 & 0.157927 \\
\hline 1 & 4.074932 & -0.818042 & 2.159103 \\
\hline 1 & 1.636117 & -0.688225 & 1.945676 \\
\hline
\end{tabular}

$\mathrm{E}(\mathrm{MeCN})=-1132.4183484$

\begin{tabular}{|c|c|c|c|c|c|c|c|}
\hline \multicolumn{4}{|c|}{ NImag $=0$} & 16 & -2.188692 & -1.835058 & 0.240811 \\
\hline & & & & 6 & -3.105086 & -0.931484 & -1.068757 \\
\hline \multirow{3}{*}{\multicolumn{4}{|c|}{$\begin{array}{l}\text { 69. TS-4R (Optimized at the mPW1PW91/6-31G* } \\
\text { level) }\end{array}$}} & 6 & -1.230441 & -3.050411 & -0.728755 \\
\hline & & & & 6 & -0.841532 & -0.447676 & 0.784513 \\
\hline & & & & 6 & -1.549725 & 0.846077 & 1.174439 \\
\hline 6 & 2.937542 & 1.887908 & 0.816542 & 6 & -1.948418 & 0.906691 & 2.651062 \\
\hline 6 & 1.693041 & 1.415705 & 0.430837 & 6 & -0.497674 & 1.826666 & 0.722218 \\
\hline 6 & 1.606188 & 0.370122 & -0.484678 & 6 & -0.714826 & 2.489790 & -0.512949 \\
\hline 6 & 2.773359 & -0.169526 & -1.024278 & 8 & -1.686677 & 2.219216 & -1.258201 \\
\hline 6 & 4.012787 & 0.298175 & -0.626479 & 6 & 0.312781 & 3.510803 & -0.986898 \\
\hline 6 & 4.094263 & 1.326736 & 0.300101 & 1 & -4.040178 & -0.587996 & -0.623258 \\
\hline 6 & 0.312973 & -0.250045 & -0.919543 & 1 & -3.318555 & -1.645091 & -1.867800 \\
\hline 6 & -0.854972 & -0.395620 & 0.063788 & 1 & -2.497242 & -0.095311 & -1.431445 \\
\hline 16 & -0.655400 & -2.071673 & 0.914109 & 1 & -1.914769 & -3.829610 & -1.073067 \\
\hline 6 & 0.745985 & -1.871618 & 2.036466 & 1 & -0.746282 & -2.533962 & -1.557530 \\
\hline 8 & 0.225896 & -0.801477 & -1.977330 & 1 & -0.483726 & -3.487895 & -0.061789 \\
\hline 6 & -2.285159 & -0.228647 & -0.581333 & 1 & -0.380618 & -0.935996 & 1.651644 \\
\hline 6 & -3.218005 & -1.439898 & -0.484088 & 1 & -2.660590 & 0.115524 & 2.921275 \\
\hline 6 & -2.934791 & 0.993447 & 0.012428 & 1 & -1.072904 & 0.804486 & 3.305514 \\
\hline
\end{tabular}




$\begin{array}{lrrr}1 & -2.417288 & 1.870669 & 2.873071 \\ 1 & -2.424626 & 1.024747 & 0.539699 \\ 1 & 0.284586 & 2.135357 & 1.408330 \\ 1 & 0.830246 & 3.111869 & -1.868156 \\ 1 & -0.198415 & 4.427794 & -1.300071 \\ 1 & 1.059560 & 3.756186 & -0.224383 \\ 6 & 0.167243 & -0.236158 & -0.360761 \\ 8 & -0.226304 & -0.277565 & -1.535888 \\ 6 & 1.637485 & -0.339146 & -0.059119 \\ 6 & 2.507107 & -0.455038 & -1.156997 \\ 6 & 3.880459 & -0.587796 & -0.969319 \\ 6 & 4.414360 & -0.619858 & 0.321211 \\ 6 & 3.561405 & -0.505575 & 1.421287 \\ 6 & 2.187833 & -0.359686 & 1.233461 \\ 1 & 2.075264 & -0.438578 & -2.151979 \\ 1 & 4.536859 & -0.670881 & -1.831895 \\ 1 & 5.485701 & -0.726670 & 0.469220 \\ 1 & 3.967397 & -0.518440 & 2.429523 \\ 1 & 1.552677 & -0.231680 & 2.105014\end{array}$

$\mathrm{E}(\mathrm{MeCN})=-1132.4149782$

NImag $=1(-98.0)$

71. PC-4 (-1132.2572074)

$\begin{array}{lrrr}16 & -3.655699 & -0.134235 & -0.805740 \\ 6 & -3.689192 & -1.410846 & 0.502675 \\ 6 & -3.623770 & 1.360046 & 0.245969 \\ 6 & 0.246693 & -1.175381 & -0.424444 \\ 6 & 0.400417 & -2.554697 & 0.102035 \\ 6 & -0.259587 & -3.742355 & -0.562142 \\ 6 & 1.604260 & -1.874530 & -0.559693 \\ 6 & 2.748043 & -1.462391 & 0.309508 \\ 8 & 2.569616 & -0.978278 & 1.415518 \\ 6 & 4.140385 & -1.689942 & -0.249821 \\ 1 & -3.714511 & -2.383553 & 0.003274 \\ 1 & -4.585985 & -1.312619 & 1.122702 \\ 1 & -2.791389 & -1.342273 & 1.123792 \\ 1 & -4.549920 & 1.453807 & 0.821973 \\ 1 & -2.765970 & 1.333119 & 0.923689 \\ 1 & -3.531540 & 2.223430 & -0.418323 \\ 1 & -0.291214 & -1.058437 & -1.365182 \\ 1 & -1.285888 & -3.866890 & -0.197200 \\ 1 & -0.306240 & -3.617871 & -1.650427 \\ 1 & 0.286124 & -4.669612 & -0.351410 \\ 1 & 0.482285 & -2.602466 & 1.186375 \\ 1 & 1.865780 & -2.219646 & -1.558097 \\ 1 & 4.888981 & -1.396306 & 0.488370 \\ 1 & 4.280086 & -2.743826 & -0.521822 \\ 1 & 4.279617 & -1.102011 & -1.166404 \\ 6 & 0.019127 & -0.011416 & 0.524042\end{array}$

$\begin{array}{lrrr}8 & -0.633315 & -0.164361 & 1.547222 \\ 6 & 0.531708 & 1.338248 & 0.133026 \\ 6 & 0.392795 & 2.395289 & 1.046349 \\ 6 & 0.843911 & 3.670823 & 0.722288 \\ 6 & 1.435219 & 3.908412 & -0.522506 \\ 6 & 1.577211 & 2.864249 & -1.438210 \\ 6 & 1.132352 & 1.583832 & -1.110334 \\ 1 & -0.066168 & 2.187854 & 2.007371 \\ 1 & 0.738193 & 4.481783 & 1.437854 \\ 1 & 1.786056 & 4.905232 & -0.776675 \\ 1 & 2.035236 & 3.046874 & -2.406563 \\ 1 & 1.249814 & 0.776241 & -1.825844\end{array}$

$\mathrm{E}(\mathrm{MeCN})=-1132.4840367$

NImag $=0$

72. TS-4'c (-1132.176964)

\begin{tabular}{crrr}
16 & 1.837503 & -1.464517 & -0.597498 \\
6 & 1.229789 & -2.537359 & -1.946652 \\
6 & 2.006411 & -2.701404 & 0.736375 \\
6 & 0.453023 & -0.412098 & -0.185993 \\
6 & 0.988434 & 0.748422 & 1.292135 \\
6 & 2.214874 & 1.374936 & 0.934036 \\
6 & 2.240968 & 2.224659 & -0.198188 \\
6 & 3.530727 & 2.957020 & -0.553067 \\
6 & 0.889912 & 0.004798 & 2.610201 \\
1 & 1.053243 & -1.890967 & -2.808799 \\
1 & 0.307473 & -3.022271 & -1.623727 \\
1 & 2.010804 & -3.264721 & -2.183257 \\
1 & 1.021304 & -3.114600 & 0.948632 \\
1 & 2.710053 & -3.459419 & 0.383174 \\
1 & 2.422278 & -2.188252 & 1.602812 \\
1 & 0.523893 & 0.435630 & -0.876776 \\
1 & 0.069974 & -0.720843 & 2.623925 \\
1 & 1.821301 & -0.514121 & 2.864984 \\
1 & 0.702569 & 0.729840 & 3.412160 \\
1 & 0.112957 & 1.370160 & 1.107230 \\
1 & 3.133970 & 1.117024 & 1.455039 \\
8 & 1.239509 & 2.389174 & -0.948655 \\
1 & 3.830216 & 2.708756 & -1.578324 \\
1 & 4.356344 & 2.725045 & 0.127957 \\
1 & 3.350141 & 4.038347 & -0.529537 \\
6 & -0.843104 & -1.066389 & -0.018278 \\
8 & -0.958252 & -2.284672 & 0.230115 \\
6 & -2.060662 & -0.195388 & -0.082239 \\
6 & -3.295651 & -0.789637 & 0.231305 \\
6 & -4.471552 & -0.049195 & 0.180278 \\
6 & -4.432576 & 1.299869 & -0.186855 \\
6 & -3.212219 & 1.899323 & -0.500760 \\
6 & -2.027528 & 1.162434 & -0.451837 \\
& & & \\
\hline
\end{tabular}




$\begin{array}{rrrr}1 & -3.301733 & -1.837580 & 0.512110 \\ 1 & -5.419942 & -0.519869 & 0.426338 \\ 1 & -5.351087 & 1.879805 & -0.228172 \\ 1 & -3.176633 & 2.946667 & -0.787854 \\ 1 & -1.092294 & 1.657676 & -0.702399\end{array}$

$\mathrm{E}(\mathrm{MeCN})=-1132.4158436$

NImag $=1(-373.4)$

73. TS-4'c conformer2 (-1132.171686)

$\begin{array}{crrr}16 & 1.379332 & 1.650935 & -0.092330 \\ 6 & 2.418689 & 1.501006 & 1.402034 \\ 6 & 0.451477 & 3.174545 & 0.359919 \\ 6 & 0.184774 & 0.316353 & 0.186381 \\ 6 & 1.091035 & -1.282212 & -0.680005 \\ 6 & 2.218639 & -1.743701 & 0.043467 \\ 6 & 3.488848 & -1.132186 & -0.118023 \\ 6 & 4.725222 & -1.859738 & 0.405167 \\ 1 & 2.517633 & 0.437792 & 1.618757 \\ 1 & 1.948600 & 2.030767 & 2.234137 \\ 1 & 3.399951 & 1.908586 & 1.161604 \\ 1 & -0.051379 & 3.050938 & 1.321989 \\ 1 & 1.172990 & 3.995318 & 0.402148 \\ 1 & -0.272416 & 3.342953 & -0.436483 \\ 1 & 0.130727 & 0.056367 & 1.239812 \\ 1 & 2.120610 & -2.633618 & 0.661638 \\ 8 & 3.649174 & -0.015628 & -0.673758 \\ 1 & 5.376674 & -2.111069 & -0.440471 \\ 1 & 4.486792 & -2.780771 & 0.946849 \\ 1 & 5.301164 & -1.194526 & 1.059711 \\ 6 & -1.040890 & 0.617153 & -0.537445 \\ 8 & -0.998335 & 1.324437 & -1.563134 \\ 6 & -2.350413 & 0.043985 & -0.078330 \\ 6 & -3.470026 & 0.261110 & -0.898277 \\ 6 & -4.725309 & -0.212288 & -0.527089 \\ 6 & -4.885111 & -0.908121 & 0.674209 \\ 6 & -3.780502 & -1.127407 & 1.499907 \\ 6 & -2.522541 & -0.655644 & 1.127444 \\ 1 & -3.324249 & 0.807560 & -1.823992 \\ 1 & -5.581484 & -0.039035 & -1.173693 \\ 1 & -5.864963 & -1.276440 & 0.966292 \\ 1 & -3.898155 & -1.666755 & 2.435983 \\ 1 & -1.678585 & -0.838417 & 1.785225 \\ 1 & 1.334780 & -0.704768 & -1.572238 \\ 6 & -0.042866 & -2.267480 & -0.887357 \\ 1 & -0.410167 & -2.677542 & 0.060217 \\ 1 & -0.889172 & -1.836468 & -1.427271 \\ 1 & 0.338450 & -3.108550 & -1.479820\end{array}$

$\mathrm{E}(\mathrm{MeCN})=-1132.4161512$

NImag $=1(-325.8)$
74. I-4'c conformer1 (-1132.185755)

$\begin{array}{lrrr}16 & -1.434013 & 1.360817 & -0.849692 \\ 6 & -0.324549 & 2.830760 & -1.155717 \\ 6 & -2.206062 & 1.886473 & 0.716052 \\ 6 & -0.170280 & 0.065019 & -0.436067 \\ 6 & -1.014542 & -1.102516 & 0.210874 \\ 6 & -2.348419 & -1.168243 & -0.465122 \\ 6 & -3.539810 & -1.042257 & 0.270121 \\ 6 & -4.856616 & -1.387829 & -0.427232 \\ 1 & 0.321733 & 2.599974 & -2.007037 \\ 1 & 0.266604 & 3.074005 & -0.271924 \\ 1 & -0.984685 & 3.660148 & -1.420613 \\ 1 & -1.432916 & 2.277920 & 1.375151 \\ 1 & -2.929826 & 2.656805 & 0.435048 \\ 1 & -2.727911 & 1.015787 & 1.159773 \\ 1 & 0.148480 & -0.244295 & -1.436338 \\ 1 & -2.380218 & -1.591365 & -1.468565 \\ 8 & -3.606520 & -0.620400 & 1.462438 \\ 1 & -5.345907 & -2.205361 & 0.116195 \\ 1 & -4.730138 & -1.687694 & -1.473227 \\ 1 & -5.536189 & -0.528197 & -0.380120 \\ 6 & 0.992463 & 0.553618 & 0.416642 \\ 8 & 0.786720 & 1.333154 & 1.343994 \\ 6 & 2.373054 & 0.033573 & 0.163449 \\ 6 & 3.370357 & 0.390704 & 1.089616 \\ 6 & 4.684650 & -0.026270 & 0.912517 \\ 6 & 5.027957 & -0.806246 & -0.196104 \\ 6 & 4.048904 & -1.166660 & -1.123348 \\ 6 & 2.729995 & -0.751064 & -0.946608 \\ 1 & 3.084322 & 0.997137 & 1.942177 \\ 1 & 5.443669 & 0.253900 & 1.637568 \\ 1 & 6.054995 & -1.132355 & -0.335884 \\ 1 & 4.310728 & -1.773484 & -1.985433 \\ 1 & 1.988424 & -1.051152 & -1.678989 \\ 1 & -1.190972 & -0.825300 & 1.256909 \\ 6 & -0.223953 & -2.420672 & 0.170677 \\ 1 & -0.811395 & -3.195382 & 0.671990 \\ 1 & -0.055475 & -2.751996 & -0.862667 \\ 1 & 0.750401 & -2.354537 & 0.671111 \\ & & & \\ 6 & & & \end{array}$

$\mathrm{E}(\mathrm{MeCN})=-1132.429134$

NImag $=0$

75. I-4'c conformer2 (-1132.18575)

$\begin{array}{cccc}16 & -1.433432 & 1.360615 & -0.849890 \\ 6 & -0.323462 & 2.830130 & -1.155949 \\ 6 & -2.205706 & 1.886952 & 0.715499 \\ 6 & -0.170300 & 0.064491 & -0.435494 \\ 6 & -1.015145 & -1.102347 & 0.211926 \\ 6 & -2.348655 & -1.168302 & -0.464753\end{array}$




\begin{tabular}{|c|c|c|c|c|c|c|c|}
\hline 6 & -3.540480 & -1.041541 & 0.269635 & 1 & -0.598499 & 2.900873 & -0.580451 \\
\hline 6 & -4.856917 & -1.387176 & -0.428388 & 1 & 0.171949 & -0.095255 & 1.091589 \\
\hline 1 & 0.322448 & 2.599260 & -2.007529 & 1 & 2.388130 & -2.569313 & 0.769019 \\
\hline 1 & 0.268060 & 3.072955 & -0.272284 & 8 & 3.364793 & 0.262255 & -0.657664 \\
\hline 1 & -0.983337 & 3.659855 & -1.420435 & 1 & 5.499478 & -1.375568 & -0.357008 \\
\hline 1 & -1.432466 & 2.277575 & 1.374981 & 1 & 4.794211 & -2.213321 & 1.046868 \\
\hline 1 & -2.928531 & 2.658059 & 0.434193 & 1 & 5.231641 & -0.485340 & 1.136799 \\
\hline 1 & -2.728624 & 1.016746 & 1.158932 & 6 & -0.944431 & 0.234958 & -0.774677 \\
\hline 1 & 0.148371 & -0.245481 & -1.435584 & 8 & -0.849295 & 0.644095 & -1.928306 \\
\hline 1 & -2.379935 & -1.591969 & -1.467982 & 6 & -2.284035 & -0.052777 & -0.173284 \\
\hline 8 & -3.607890 & -0.618943 & 1.461654 & 6 & -3.407734 & 0.132579 & -0.999249 \\
\hline 1 & -5.347034 & -2.203895 & 0.115513 & 6 & -4.690702 & -0.087546 & -0.511034 \\
\hline 1 & -4.729792 & -1.688166 & -1.473980 & 6 & -4.875085 & -0.496638 & 0.813441 \\
\hline 1 & -5.536105 & -0.527159 & -0.382684 & 6 & -3.768770 & -0.686220 & 1.643017 \\
\hline 6 & 0.992585 & 0.553021 & 0.417069 & 6 & -2.481050 & -0.466707 & 1.154946 \\
\hline 8 & 0.786906 & 1.332474 & 1.344516 & 1 & -3.243093 & 0.451907 & -2.022849 \\
\hline 6 & 2.373183 & 0.033209 & 0.163529 & 1 & -5.549420 & 0.059098 & -1.160303 \\
\hline 6 & 3.370952 & 0.391586 & 1.088718 & 1 & -5.877454 & -0.667635 & 1.196556 \\
\hline 6 & 4.685272 & -0.025113 & 0.911196 & 1 & -3.906833 & -1.007227 & 2.671653 \\
\hline 6 & 5.028132 & -0.806128 & -0.196835 & 1 & -1.637942 & -0.628955 & 1.817951 \\
\hline 6 & 4.048604 & -1.167847 & -1.123067 & 1 & 1.296401 & -1.061585 & -1.564237 \\
\hline 6 & 2.729678 & -0.752471 & -0.945938 & 6 & 0.090418 & -2.498580 & -0.500456 \\
\hline 1 & 3.085246 & 0.998762 & 1.940861 & 1 & 0.617780 & -3.379708 & -0.880218 \\
\hline 1 & 5.444665 & 0.256071 & 1.635462 & 1 & -0.247424 & -2.731810 & 0.517297 \\
\hline 1 & 6.055191 & -1.132034 & -0.336934 & 1 & -0.794052 & -2.340900 & -1.126364 \\
\hline 1 & 4.310069 & -1.775518 & -1.984664 & & & & \\
\hline 1 & 1.987732 & -1.053596 & -1.677510 & \multicolumn{4}{|c|}{$\mathrm{E}(\mathrm{MeCN})=-1132.4241812$} \\
\hline 1 & -1.192025 & -0.824288 & 1.257658 & \multicolumn{4}{|c|}{ NImag $=0$} \\
\hline 6 & -0.224760 & -2.420668 & 0.173163 & & & & \\
\hline 1 & -0.812623 & -3.194905 & 0.674714 & \multicolumn{4}{|c|}{ 77. TS-4't (-1132.17911) } \\
\hline 1 & -0.055731 & -2.752804 & -0.859831 & & & & \\
\hline \multirow[t]{2}{*}{1} & 0.749317 & -2.354317 & 0.674119 & 6 & -1.910278 & 0.895097 & -0.672638 \\
\hline & & & & 6 & -1.959671 & -0.390449 & -0.107531 \\
\hline \multicolumn{4}{|c|}{$\mathrm{E}(\mathrm{MeCN})=-1132.4289696$} & 6 & -3.210423 & -0.959267 & 0.190702 \\
\hline \multirow{2}{*}{\multicolumn{4}{|c|}{ NImag $=0$}} & 6 & -4.384295 & -0.262872 & -0.074247 \\
\hline & & & & 6 & -4.327227 & 1.016340 & -0.637753 \\
\hline \multirow{2}{*}{\multicolumn{4}{|c|}{ 76. I-4'c conformer3 (-1132.1832801) }} & 6 & -3.090699 & 1.593360 & -0.929936 \\
\hline & & & & 6 & -0.742913 & -1.198977 & 0.193966 \\
\hline 16 & 1.386945 & 1.536509 & -0.207773 & 8 & -0.842553 & -2.329103 & 0.713636 \\
\hline 6 & 2.308668 & 1.622465 & 1.369082 & 6 & 0.577110 & -0.626941 & -0.066616 \\
\hline 6 & 0.066445 & 2.768819 & 0.273293 & 16 & 1.810154 & -1.945149 & 0.025504 \\
\hline 6 & 0.332253 & -0.014681 & 0.014945 & 6 & 1.313525 & -3.271713 & -1.135591 \\
\hline 6 & 1.064581 & -1.307833 & -0.517637 & 6 & 3.197405 & -1.189663 & -0.890352 \\
\hline 6 & 2.331556 & -1.638454 & 0.211790 & 6 & 1.114452 & 0.661224 & 1.330551 \\
\hline 6 & 3.440232 & -0.861405 & -0.017200 & 6 & 0.991488 & 1.989521 & 0.843106 \\
\hline 6 & 4.812974 & -1.269202 & 0.491999 & 6 & 1.921173 & 2.492601 & -0.097873 \\
\hline 1 & 2.445453 & 0.596853 & 1.720645 & 8 & 2.840831 & 1.800723 & -0.614195 \\
\hline 1 & 1.758341 & 2.223241 & 2.096446 & 6 & 0.344645 & 0.262711 & 2.574891 \\
\hline 1 & 3.281224 & 2.050692 & 1.133028 & 6 & 1.817073 & 3.954246 & -0.523970 \\
\hline 1 & -0.500591 & 2.455776 & 1.154840 & 1 & 3.227972 & -0.118069 & -0.627922 \\
\hline 1 & 0.583675 & 3.710568 & 0.477019 & 1 & 4.112294 & -1.712699 & -0.604729 \\
\hline
\end{tabular}




$\begin{array}{rrrr}1 & 3.018802 & -1.288817 & -1.963965 \\ 1 & 0.739652 & -0.024904 & -0.955740 \\ 1 & 2.132373 & -3.994448 & -1.181551 \\ 1 & 1.117581 & -2.844841 & -2.121671 \\ 1 & 0.414413 & -3.722364 & -0.717303 \\ 1 & 2.151913 & 0.319244 & 1.349452 \\ 1 & 0.354167 & -0.817843 & 2.748711 \\ 1 & -0.699361 & 0.588597 & 2.519854 \\ 1 & 0.798336 & 0.751029 & 3.446171 \\ 1 & 0.152580 & 2.601100 & 1.163606 \\ 1 & 2.744979 & 4.474812 & -0.258301 \\ 1 & 1.721618 & 4.016405 & -1.614737 \\ 1 & 0.974946 & 4.478277 & -0.060267 \\ 1 & -3.229928 & -1.949432 & 0.633692 \\ 1 & -5.345807 & -0.712772 & 0.158628 \\ 1 & -5.244614 & 1.560112 & -0.847898 \\ 1 & -3.042258 & 2.589382 & -1.361655 \\ 1 & -0.956529 & 1.365375 & -0.888221\end{array}$

$\mathrm{E}(\mathrm{MeCN})=-1132.4172092$

NImag $=1(-330.3)$

78. TS-4' $t$ conformer2 (-1132.17078)

$\begin{array}{crrr}6 & -3.377236 & 0.873301 & -0.661078 \\ 6 & -2.036373 & 0.494431 & -0.574435 \\ 6 & -1.692197 & -0.796218 & -0.137374 \\ 6 & -2.719019 & -1.696769 & 0.201627 \\ 6 & -4.052733 & -1.317040 & 0.109573 \\ 6 & -4.386167 & -0.028614 & -0.323743 \\ 6 & -0.283747 & -1.281115 & -0.023669 \\ 6 & 0.791168 & -0.295835 & -0.191255 \\ 6 & 1.021992 & 0.935611 & 1.286561 \\ 6 & 0.496898 & 0.260825 & 2.541526 \\ 8 & -0.056996 & -2.474368 & 0.264071 \\ 16 & 2.424329 & -0.977147 & -0.532108 \\ 6 & 2.189928 & -2.258236 & -1.815862 \\ 6 & 2.857982 & -2.029432 & 0.895071 \\ 6 & 0.470108 & 2.203065 & 0.938676 \\ 6 & 0.993408 & 2.895863 & -0.178870 \\ 6 & 0.488975 & 4.302214 & -0.491379 \\ 8 & 1.845590 & 2.378418 & -0.950522 \\ 1 & 1.901994 & -1.737160 & -2.731264 \\ 1 & 3.146727 & -2.765094 & -1.967135 \\ 1 & 1.410688 & -2.948181 & -1.490537 \\ 1 & 0.703573 & 0.485569 & -0.944547 \\ 1 & 3.804962 & -2.519650 & 0.655955 \\ 1 & 2.050552 & -2.739392 & 1.067392 \\ 1 & 2.995968 & -1.370296 & 1.753307 \\ 1 & 2.113802 & 0.926230 & 1.210029 \\ 1 & 0.843244 & -0.771614 & 2.652527\end{array}$

$\begin{array}{rrrr}1 & -0.597732 & 0.244888 & 2.546856 \\ 1 & 0.824897 & 0.826221 & 3.422570 \\ 1 & -0.414877 & 2.564552 & 1.455330 \\ 1 & 1.329116 & 5.005658 & -0.443945 \\ 1 & 0.102378 & 4.341268 & -1.516947 \\ 1 & -0.289696 & 4.641942 & 0.199826 \\ 1 & -2.437861 & -2.689436 & 0.537031 \\ 1 & -4.836481 & -2.021490 & 0.375753 \\ 1 & -5.429298 & 0.267588 & -0.399123 \\ 1 & -3.630504 & 1.874822 & -0.997982 \\ 1 & -1.267384 & 1.213219 & -0.831986\end{array}$

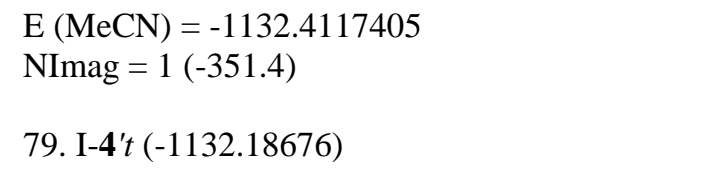

$\begin{array}{crrr}16 & 2.120177 & -1.698568 & 0.070374 \\ 6 & 3.588597 & -0.620818 & -0.176718 \\ 6 & 1.948899 & -2.360319 & -1.620170 \\ 6 & 0.735478 & -0.429024 & 0.124741 \\ 6 & 0.979643 & 0.551649 & 1.361722 \\ 6 & 0.760276 & 1.971353 & 0.939790 \\ 6 & 1.321070 & 2.479654 & -0.220047 \\ 6 & 1.064716 & 3.934347 & -0.597512 \\ 1 & 3.243514 & 0.345696 & -0.585053 \\ 1 & 4.269504 & -1.153112 & -0.845897 \\ 1 & 4.065301 & -0.490776 & 0.795834 \\ 1 & 1.906699 & -1.531369 & -2.331142 \\ 1 & 2.815419 & -2.994811 & -1.819764 \\ 1 & 1.033819 & -2.950574 & -1.639748 \\ 1 & 0.925607 & 0.186396 & -0.770425 \\ 1 & 0.136970 & 2.594979 & 1.573492 \\ 8 & 2.029897 & 1.790394 & -1.050436 \\ 1 & 2.021472 & 4.464000 & -0.686522 \\ 1 & 0.437455 & 4.462674 & 0.128924 \\ 1 & 0.586915 & 3.983602 & -1.584353 \\ 6 & -0.529544 & -1.257353 & 0.105231 \\ 8 & -0.443021 & -2.484314 & 0.246506 \\ 6 & -1.846319 & -0.604802 & -0.097532 \\ 6 & -2.997552 & -1.402099 & 0.055266 \\ 6 & -4.259967 & -0.857464 & -0.139569 \\ 6 & -4.392594 & 0.491107 & -0.493603 \\ 6 & -3.258242 & 1.287480 & -0.647897 \\ 6 & -1.985589 & 0.746376 & -0.457591 \\ 1 & -2.872175 & -2.443927 & 0.330739 \\ 1 & -5.143542 & -1.477730 & -0.015884 \\ 1 & -5.380780 & 0.915909 & -0.650542 \\ 1 & -3.358984 & 2.333713 & -0.922740 \\ 1 & -1.107600 & 1.376766 & -0.556477 \\ 1 & 2.042639 & 0.399771 & 1.618230\end{array}$




\begin{tabular}{|c|c|c|c|}
\hline 6 & 0.176636 & 0.154449 & 2.608357 \\
\hline 1 & 0.487696 & 0.782471 & 3.450129 \\
\hline 1 & -0.896695 & 0.311105 & 2.460510 \\
\hline 1 & 0.335878 & -0.893553 & 2.889349 \\
\hline \multicolumn{4}{|c|}{$E(\mathrm{MeCN})=-1132.4253641$} \\
\hline \multicolumn{4}{|c|}{ 80. TS-4'R (-1132.17822) } \\
\hline 6 & 2.673389 & -1.175079 & -1.729749 \\
\hline 1 & 1.947508 & -1.420049 & -2.509673 \\
\hline 1 & 2.838572 & -0.090831 & -1.633138 \\
\hline 16 & 2.017414 & -1.681975 & -0.105223 \\
\hline 6 & 1.342720 & -3.325723 & -0.545791 \\
\hline 1 & 0.580300 & -3.244411 & -1.321745 \\
\hline 1 & 0.913157 & -3.769449 & 0.348582 \\
\hline 6 & 0.621085 & -0.427936 & 0.157506 \\
\hline 1 & 0.701855 & 0.173491 & -0.743844 \\
\hline 6 & 1.043732 & 0.528109 & 1.403785 \\
\hline 6 & 0.219942 & 0.258590 & 2.668273 \\
\hline 1 & 0.639515 & 0.847846 & 3.490205 \\
\hline 1 & -0.825523 & 0.563758 & 2.546262 \\
\hline 1 & 0.232644 & -0.796402 & 2.962995 \\
\hline 6 & 1.030224 & 1.945019 & 0.951868 \\
\hline 1 & 0.370981 & 2.661342 & 1.432264 \\
\hline 6 & 1.944498 & 2.305831 & -0.025743 \\
\hline 6 & 2.045149 & 3.753175 & -0.491163 \\
\hline 1 & 1.333149 & 4.414396 & 0.015226 \\
\hline 1 & 3.061059 & 4.126319 & -0.311082 \\
\hline 1 & 1.876090 & 3.813168 & -1.574126 \\
\hline 8 & 2.732697 & 1.452295 & -0.572911 \\
\hline 6 & -0.726600 & -1.101370 & 0.323542 \\
\hline 8 & -0.817003 & -2.225821 & 0.818591 \\
\hline 1 & 2.198219 & -3.904299 & -0.905442 \\
\hline 1 & 3.609023 & -1.716923 & -1.885997 \\
\hline 6 & -1.941447 & -0.333969 & -0.071318 \\
\hline 1 & 2.083460 & 0.229889 & 1.605680 \\
\hline 6 & -1.897112 & 0.988021 & -0.547957 \\
\hline 6 & -3.080402 & 1.642287 & -0.893285 \\
\hline 6 & -4.306266 & 0.984845 & -0.786637 \\
\hline 6 & -4.356817 & -0.331075 & -0.311702 \\
\hline 6 & -3.184628 & -0.983205 & 0.049653 \\
\hline 1 & -0.951659 & 1.523191 & -0.597761 \\
\hline 1 & -3.041002 & 2.668047 & -1.249123 \\
\hline 1 & -5.223272 & 1.494985 & -1.070116 \\
\hline 1 & -5.311978 & -0.841122 & -0.220984 \\
\hline 1 & -3.201071 & -1.998270 & 0.432258 \\
\hline
\end{tabular}

81. TS-4'E (-1132.18090)

$\begin{array}{crrr}6 & -2.106891 & 0.581044 & -0.662409 \\ 6 & -1.837957 & -0.642217 & -0.029295 \\ 6 & -2.909423 & -1.480760 & 0.330910 \\ 6 & -4.221063 & -1.093220 & 0.083145 \\ 6 & -4.480694 & 0.127756 & -0.548751 \\ 6 & -3.424038 & 0.958087 & -0.924775 \\ 6 & -0.457964 & -1.155175 & 0.224515 \\ 8 & -0.293008 & -2.345497 & 0.486392 \\ 6 & 0.765384 & -0.245370 & 0.155438 \\ 16 & 2.370012 & -1.692379 & -0.078201 \\ 6 & 1.911808 & -2.398362 & -1.694697 \\ 6 & 3.667880 & -0.505878 & -0.596656 \\ 6 & 1.113724 & 0.641722 & 1.346638 \\ 6 & 0.437778 & 1.900708 & 0.907552 \\ 6 & 1.039667 & 2.656746 & -0.113266 \\ 8 & 2.095739 & 2.293153 & -0.713794 \\ 6 & 0.401424 & 3.978344 & -0.534149 \\ 1 & 4.216876 & -0.925687 & -1.443348 \\ 1 & 3.210814 & 0.463164 & -0.843863 \\ 1 & 1.616136 & -1.605165 & -2.387936 \\ 1 & 1.078477 & -3.079276 & -1.520364 \\ 1 & 0.962150 & 0.261363 & -0.787621 \\ 1 & -0.488170 & 2.206258 & 1.383502 \\ 1 & -0.516496 & 4.208377 & 0.017997 \\ 1 & 1.118137 & 4.793400 & -0.377442 \\ 1 & 0.177602 & 3.958979 & -1.608144 \\ 1 & 2.765539 & -2.947228 & -2.099349 \\ 1 & 4.350105 & -0.384325 & 0.247160 \\ 1 & -2.687215 & -2.430380 & 0.805779 \\ 1 & -5.042552 & -1.740137 & 0.378379 \\ 1 & -5.505974 & 0.429038 & -0.747392 \\ 1 & -3.623027 & 1.904150 & -1.420265 \\ 1 & -1.287543 & 1.239104 & -0.924947 \\ 6 & 0.672539 & 0.090185 & 2.703345 \\ 1 & -0.418483 & -0.000672 & 2.765044 \\ 1 & 1.103241 & -0.897381 & 2.905541 \\ 1 & 0.990934 & 0.773778 & 3.496955 \\ 1 & 2.204344 & 0.787804 & 1.345485 \\ & & & \\ 6 & & \end{array}$

$\mathrm{E}(\mathrm{MeCN})=-1132.4149782$

NImag $=1(-226.8)$

82. PC-4' (-1132.26580)

$\begin{array}{lrrr}6 & -2.551454 & -1.375098 & -0.340034 \\ 6 & -2.858950 & -0.046962 & -0.006354 \\ 6 & -4.206340 & 0.344675 & 0.061348 \\ 6 & -5.221803 & -0.565959 & -0.210870 \\ 6 & -4.905657 & -1.886609 & -0.544510 \\ 6 & -3.570783 & -2.290037 & -0.603447\end{array}$




\begin{tabular}{|c|c|c|c|c|c|c|c|}
\hline 6 & -1.817488 & 0.984491 & 0.307383 & 6 & -1.863147 & -0.564364 & 0.738658 \\
\hline 8 & -2.143972 & 2.067134 & 0.785205 & 1 & -2.131353 & -0.216973 & 1.734111 \\
\hline 6 & -0.380884 & 0.663901 & 0.033416 & 6 & -2.625873 & -0.180369 & -0.398405 \\
\hline 16 & 3.871216 & -1.921522 & 0.803090 & 6 & -3.940259 & 0.568079 & -0.189455 \\
\hline 6 & 2.884849 & -2.587013 & -0.584669 & 1 & -4.165158 & 0.757517 & 0.865642 \\
\hline 6 & 4.983182 & -0.816518 & -0.138007 & 1 & -3.913424 & 1.522969 & -0.727869 \\
\hline 6 & 0.674263 & 0.820343 & 1.114765 & 1 & -4.760168 & -0.016775 & -0.622898 \\
\hline 6 & 0.604658 & 1.831561 & -0.010344 & 8 & -2.250075 & -0.414635 & -1.573177 \\
\hline 6 & 1.661408 & 1.803463 & -1.062974 & 6 & 1.962524 & -0.772139 & -0.481564 \\
\hline 8 & 2.123421 & 0.752523 & -1.487874 & 8 & 2.899483 & -0.252355 & 0.147234 \\
\hline 6 & 2.143685 & 3.146446 & -1.573605 & 1 & 0.982771 & 2.583728 & 2.009257 \\
\hline 1 & 5.641978 & -1.391770 & -0.796393 & 1 & 1.595737 & 3.401824 & -0.496290 \\
\hline 1 & 4.401888 & -0.101362 & -0.726262 & 6 & 2.184433 & -2.057589 & -1.268357 \\
\hline 1 & 3.515112 & -3.149875 & -1.280593 & 1 & 1.320458 & -2.730187 & -1.224091 \\
\hline 1 & 2.379650 & -1.773119 & -1.111593 & 1 & 2.352782 & -1.817377 & -2.326348 \\
\hline 1 & -0.174271 & -0.115689 & -0.691630 & 1 & 3.072979 & -2.564700 & -0.884162 \\
\hline 1 & 0.170155 & 2.795929 & 0.237632 & & & & \\
\hline 1 & 2.527594 & 3.753366 & -0.743829 & \multicolumn{4}{|c|}{$\mathrm{E}(\mathrm{MeCN})=-940.6324255$} \\
\hline 1 & 2.926531 & 3.004470 & -2.321123 & \multicolumn{4}{|c|}{ NImag = $1(-360.2)$} \\
\hline 1 & 1.308203 & 3.705139 & -2.014297 & & & & \\
\hline 1 & 2.141844 & -3.266363 & -0.157360 & \multirow{2}{*}{\multicolumn{4}{|c|}{ 84. TS-5 $c$ conformer2 (-940.4344539) }} \\
\hline 1 & 5.596340 & -0.277443 & 0.589398 & & & $0.4344539)$ & \\
\hline 1 & -4.428667 & 1.372036 & 0.330185 & & & & \\
\hline 1 & -6.260513 & -0.250569 & -0.162318 & 6 & -0.648169 & 2.102616 & -1.006594 \\
\hline 1 & -5.698418 & -2.599414 & -0.755678 & 1 & -0.018017 & 2.310935 & -1.875562 \\
\hline 1 & -3.322008 & -3.317472 & -0.854305 & 1 & -1.466753 & 1.402962 & -1.249225 \\
\hline 1 & -1.519508 & -1.708319 & -0.377977 & 16 & 0.357937 & 1.347097 & 0.308385 \\
\hline 6 & 0.346486 & 1.217269 & 2.537376 & 6 & 1.756538 & 2.532155 & 0.340342 \\
\hline 1 & -0.490373 & 1.918109 & 2.581998 & 1 & 2.152907 & 2.692997 & -0.664903 \\
\hline 1 & 0.088312 & 0.332346 & 3.131022 & 1 & 2.511694 & 2.102424 & 0.998608 \\
\hline 1 & 1.219275 & 1.686879 & 3.005654 & 6 & 1.041777 & -0.095790 & -0.536077 \\
\hline \multirow[t]{2}{*}{1} & 1.490821 & 0.106493 & 1.012534 & 1 & 1.153198 & 0.089306 & -1.604713 \\
\hline & & & & 6 & -0.477064 & -1.378175 & -0.437033 \\
\hline \multicolumn{4}{|c|}{$\mathrm{E}(\mathrm{MeCN})=-1132.4900764$} & 6 & 0.213670 & -2.703972 & -0.176571 \\
\hline \multirow{2}{*}{\multicolumn{4}{|c|}{ NImag $=0$}} & 1 & 0.643548 & -2.741287 & 0.830624 \\
\hline & & & & 1 & -0.533582 & -3.503995 & -0.244907 \\
\hline \multirow{2}{*}{\multicolumn{4}{|c|}{ 83. TS-5c (-940.4338199) }} & 1 & 1.002881 & -2.923022 & -0.902759 \\
\hline & & & & 1 & -0.783853 & -1.238661 & -1.473968 \\
\hline 6 & 1.628473 & 2.368154 & -0.850682 & 6 & -1.491645 & -0.982022 & 0.480291 \\
\hline 1 & 2.598064 & 1.905310 & -0.660815 & 1 & -1.422022 & -1.318377 & 1.512183 \\
\hline 1 & 1.377883 & 2.337888 & -1.913304 & 6 & -2.682992 & -0.340770 & 0.044126 \\
\hline 16 & 0.329154 & 1.426463 & 0.024520 & 6 & -3.840727 & -0.236825 & 1.036463 \\
\hline 6 & 0.953491 & 1.526258 & 1.735208 & 1 & -3.580532 & -0.574731 & 2.044653 \\
\hline 1 & 1.938156 & 1.061373 & 1.774621 & 1 & -4.193507 & 0.800088 & 1.082274 \\
\hline 1 & 0.230323 & 1.002343 & 2.359554 & 1 & -4.680624 & -0.839710 & 0.670308 \\
\hline 6 & 0.598248 & -0.270258 & -0.493982 & 8 & -2.862055 & 0.125598 & -1.110940 \\
\hline 1 & -0.007280 & -0.386201 & -1.396478 & 6 & 2.234409 & -0.550801 & 0.175122 \\
\hline 6 & -0.688649 & -1.342478 & 0.547232 & 8 & 2.422659 & -0.276667 & 1.367360 \\
\hline 6 & 0.007057 & -1.936207 & 1.756536 & 1 & 1.381452 & 3.470846 & 0.756557 \\
\hline 1 & 0.075860 & -1.228923 & 2.590371 & 1 & -1.057703 & 3.031551 & -0.602129 \\
\hline 1 & -0.578986 & -2.793429 & 2.111788 & 6 & 3.246291 & -1.396596 & -0.585408 \\
\hline 1 & 1.017218 & -2.293969 & 1.533547 & 1 & 2.925960 & -1.654751 & -1.598422 \\
\hline 1 & -0.765380 & -2.046437 & -0.282543 & 1 & 4.187879 & -0.836885 & -0.647137 \\
\hline
\end{tabular}




\begin{tabular}{|c|c|c|c|c|c|c|c|}
\hline 1 & 3.452712 & -2.313613 & -0.024975 & 1 & 0.101101 & -0.308314 & $\begin{array}{r}-1.428214 \\
0.323845\end{array}$ \\
\hline & & & & 6 & -0.252962 & -1.455567 & 0.323845 \\
\hline E $(N$ & 40.633133 & & & 6 & 0.442415 & -1.985632 & 1.586212 \\
\hline NIm & 20.1) & & & 1 & 0.541816 & -1.214555 & 2.357867 \\
\hline & & & & 1 & -0.161930 & -2.793698 & 2.012364 \\
\hline 85.1 & mer1 $(-940$ & 4477665) & & 1 & 1.441538 & -2.390031 & 1.385086 \\
\hline & & & & 1 & -0.273209 & -2.277234 & -0.415526 \\
\hline 6 & -1.016674 & 2.163133 & -0.415851 & 6 & -1.637924 & -0.945951 & 0.605330 \\
\hline 1 & -1.418255 & 1.433796 & -1.133287 & 1 & -2.097530 & -1.243485 & 1.544151 \\
\hline 1 & -1.804422 & 2.477830 & 0.269462 & 6 & -2.411812 & -0.381305 & -0.413946 \\
\hline 16 & 0.255925 & 1.327607 & 0.604077 & 6 & -3.868480 & -0.028935 & -0.127291 \\
\hline 6 & 1.679188 & 2.430326 & 0.154667 & 1 & -4.189380 & -0.303768 & 0.883461 \\
\hline 1 & 1.844554 & 2.444514 & -0.925294 & 1 & -4.022668 & 1.048032 & -0.270168 \\
\hline 1 & 2.564807 & 2.062432 & 0.666715 & 1 & -4.514454 & -0.538172 & -0.852769 \\
\hline 6 & 0.648386 & -0.264245 & -0.304883 & 8 & -1.950179 & -0.064924 & -1.559506 \\
\hline 1 & 0.106620 & -0.184449 & -1.256302 & 6 & 2.051413 & -0.504106 & -0.457907 \\
\hline 6 & -0.145345 & -1.357601 & 0.500491 & 8 & 2.846575 & 0.227565 & 0.117196 \\
\hline 6 & 0.564437 & -1.798155 & 1.785479 & 1 & 0.527055 & 2.693051 & 2.019934 \\
\hline 1 & 0.683204 & -0.963245 & 2.486604 & 1 & 0.649851 & 3.467424 & -0.556846 \\
\hline 1 & -0.040739 & -2.561533 & 2.286037 & 6 & 2.529463 & -1.682625 & -1.287856 \\
\hline 1 & 1.557301 & -2.222483 & 1.599094 & 1 & 1.942522 & -2.585966 & -1.091026 \\
\hline 1 & -0.186982 & -2.209982 & -0.197465 & 1 & 2.406792 & -1.449669 & -2.353611 \\
\hline 6 & -1.528962 & -0.827705 & 0.761373 & 1 & 3.586441 & -1.864394 & -1.081284 \\
\hline 1 & -1.927730 & -0.949301 & 1.764919 & & & & \\
\hline 6 & -2.388457 & -0.557518 & -0.306843 & & $32.0+4 J J^{2}$ & & \\
\hline 6 & -3.859762 & -0.263984 & -0.019711 & & & & \\
\hline 1 & -4.108269 & -0.299068 & 1.046558 & & & & \\
\hline 1 & -4.134291 & 0.720853 & -0.419179 & & ner3 $(-94$ & 475009) & \\
\hline
\end{tabular}

$\begin{array}{llll}-4.480655 & -0.997316 & -0.548633\end{array}$

$\begin{array}{llll}-2.002748 & -0.479504 & -1.520592\end{array}$

$\begin{array}{llll}2.120807 & -0.584871 & -0.471163\end{array}$

$\begin{array}{lll}2.999884 & -0.120738 & 0.236939\end{array}$

$\begin{array}{llll}1.405878 & 3.427588 & 0.508258\end{array}$

$\begin{array}{llll}-0.569663 & 3.022313 & -0.920148\end{array}$

$\begin{array}{llll}2.422114 & -1.613263 & -1.545404\end{array}$

$\begin{array}{llll}1.814364 & -2.514597 & -1.403706\end{array}$

$\begin{array}{llll}2.165101 & -1.213351 & -2.534104\end{array}$

$\begin{array}{llll}3.482904 & -1.870494 & -1.514471\end{array}$

$\mathrm{E}(\mathrm{MeCN})=-940.6447126$

NImag $=0$

86. I-5c conformer2 (-940.44621)

$\begin{array}{crrr}6 & 0.943785 & 2.449879 & -0.826639 \\ 1 & 2.007946 & 2.287381 & -0.654034 \\ 1 & 0.677693 & 2.269447 & -1.871031 \\ 16 & -0.102069 & 1.307682 & 0.177252 \\ 6 & 0.657918 & 1.627019 & 1.813491 \\ 1 & 1.711757 & 1.353285 & 1.802767 \\ 1 & 0.091510 & 1.042449 & 2.538804 \\ 6 & 0.552032 & -0.328745 & -0.426487\end{array}$

$\begin{array}{crrr}6 & 0.462314 & 2.492501 & -0.742983 \\ 1 & 1.491098 & 2.353577 & -1.075800 \\ 1 & -0.264379 & 2.380307 & -1.546695 \\ 16 & 0.011918 & 1.220866 & 0.485583 \\ 6 & 1.354627 & 1.564245 & 1.717090 \\ 1 & 2.339378 & 1.604948 & 1.256626 \\ 1 & 1.315659 & 0.777059 & 2.472672 \\ 6 & 0.516411 & -0.360244 & -0.370252 \\ 1 & 0.014036 & -0.167963 & -1.329084 \\ 6 & -0.269126 & -1.535137 & 0.271085 \\ 6 & 0.277325 & -1.957107 & 1.649488 \\ 1 & 0.102372 & -1.177838 & 2.399959 \\ 1 & -0.252876 & -2.851069 & 1.994976 \\ 1 & 1.350139 & -2.193587 & 1.632106 \\ 1 & -0.073554 & -2.382616 & -0.412728 \\ 6 & -1.750902 & -1.241082 & 0.314427 \\ 1 & -2.344120 & -1.965335 & 0.867009 \\ 6 & -2.396303 & -0.233634 & -0.362927 \\ 6 & -3.912810 & -0.138533 & -0.285514 \\ 1 & -4.365040 & -0.945891 & 0.300358 \\ 1 & -4.202265 & 0.824709 & 0.154086 \\ 1 & -4.333868 & -0.160278 & -1.298594 \\ 8 & -1.813180 & 0.710565 & -1.049060\end{array}$




\begin{tabular}{|c|c|c|c|c|c|c|c|}
\hline 6 & 1.998808 & -0.557484 & -0.654691 & 1 & -2.380699 & 2.411414 & 0.656513 \\
\hline 8 & 2.838092 & 0.315957 & -0.502266 & 16 & -2.258130 & 0.234955 & -0.355079 \\
\hline 1 & 1.091500 & 2.516761 & 2.186609 & 6 & -3.372812 & -1.162294 & 0.114665 \\
\hline 1 & 0.340984 & 3.463619 & -0.256168 & 1 & -3.345165 & -1.313920 & 1.196124 \\
\hline 6 & 2.381537 & -1.891658 & -1.274286 & 1 & -2.994598 & -2.036301 & -0.412660 \\
\hline 1 & 2.283002 & -2.701834 & -0.542976 & 6 & -0.590759 & 0.000685 & 0.354216 \\
\hline 1 & 1.728266 & -2.140195 & -2.118316 & 1 & -0.605095 & 0.255314 & 1.415916 \\
\hline 1 & 3.418396 & -1.831604 & -1.611369 & 6 & 0.715358 & 1.179519 & -0.407492 \\
\hline & & & & 6 & 0.060300 & 2.550999 & -0.459083 \\
\hline E ( & 40.6435426 & & & 1 & -0.094162 & 2.971133 & 0.543219 \\
\hline NIn & & & & 1 & 0.731397 & 3.234599 & -0.991400 \\
\hline & & & & 1 & -0.894049 & 2.558676 & -1.002157 \\
\hline 88. & $.4250486)$ & & & 1 & 0.763991 & 0.691298 & -1.381975 \\
\hline & & & & 6 & 1.945255 & 1.088778 & 0.300224 \\
\hline 6 & 3.439747 & -0.847139 & -0.401723 & 1 & 2.157291 & 1.781684 & 1.111759 \\
\hline 1 & 3.035890 & -1.713530 & 0.120425 & 6 & 2.911343 & 0.129430 & -0.110985 \\
\hline 1 & 3.548958 & -1.040767 & -1.470738 & 6 & 4.297340 & 0.168704 & 0.536746 \\
\hline 16 & 2.262395 & 0.562275 & -0.231170 & 1 & 4.398923 & 0.949475 & 1.298544 \\
\hline 6 & 2.219739 & 0.698119 & 1.591557 & 1 & 4.519475 & -0.804429 & 0.991153 \\
\hline 1 & 1.829248 & -0.235601 & 1.995074 & 1 & 5.053260 & 0.333666 & -0.240167 \\
\hline 1 & 1.571093 & 1.537172 & 1.844569 & 8 & 2.683742 & -0.742078 & -0.979698 \\
\hline 6 & 0.577817 & 0.057283 & -0.673973 & 6 & -0.203028 & -1.397696 & 0.020091 \\
\hline 1 & 0.507707 & 0.216438 & -1.751273 & 8 & -0.741479 & -1.969338 & -0.931682 \\
\hline 6 & -0.672390 & 1.320813 & -0.053887 & 1 & -4.382405 & -0.906913 & -0.218634 \\
\hline 6 & -0.163292 & 2.665839 & -0.559386 & 1 & -3.992699 & 1.671447 & 0.466107 \\
\hline 1 & -0.244283 & 2.737756 & -1.650761 & 6 & 0.784946 & -2.088009 & 0.928383 \\
\hline 1 & -0.784953 & 3.461364 & -0.133611 & 1 & 1.474691 & -1.374163 & 1.383878 \\
\hline 1 & 0.878044 & 2.883301 & -0.289312 & 1 & 0.219968 & -2.581439 & 1.733441 \\
\hline 1 & -0.514115 & 1.152981 & 1.012856 & 1 & 1.335824 & -2.841725 & 0.365323 \\
\hline 6 & -2.006788 & 0.981256 & -0.431050 & & & & \\
\hline 1 & -2.460931 & 1.443030 & -1.304579 & \multicolumn{4}{|c|}{$\mathrm{E}(\mathrm{MeCN})=-940.6244492$} \\
\hline 6 & -2.712064 & 0.041308 & 0.360300 & \multicolumn{4}{|c|}{ NImag $=1(-364.9)$} \\
\hline 6 & -4.198208 & -0.190280 & 0.086729 & & & & \\
\hline 1 & -4.584737 & 0.416526 & -0.739551 & \multicolumn{4}{|c|}{ 90. I-5t (-940.43826) } \\
\hline 1 & -4.374370 & -1.249794 & -0.135096 & & & & \\
\hline 1 & -4.771601 & 0.042000 & 0.992107 & 6 & 3.316451 & -0.272452 & -0.420756 \\
\hline 8 & -2.159317 & -0.616081 & 1.274971 & 1 & 2.940543 & -0.245925 & -1.443416 \\
\hline 6 & 0.263057 & -1.348743 & -0.243420 & 1 & 3.783359 & 0.675961 & -0.144863 \\
\hline 8 & 0.870759 & -1.902943 & 0.671552 & 16 & 1.918317 & -0.565278 & 0.715548 \\
\hline 1 & 3.239413 & 0.902579 & 1.925801 & 6 & 1.159433 & -2.090544 & 0.062912 \\
\hline 1 & 4.398007 & -0.524746 & 0.014884 & 1 & 1.277531 & -2.105507 & -1.019098 \\
\hline 6 & -0.769818 & -2.067710 & -1.078390 & 1 & 0.072828 & -2.061984 & 0.318601 \\
\hline 1 & -1.571615 & -1.385381 & -1.377058 & 6 & 0.726627 & 0.813136 & 0.361325 \\
\hline 1 & -0.288400 & -2.441099 & -1.994023 & 1 & 1.322353 & 1.697085 & 0.644525 \\
\hline \multirow[t]{2}{*}{1} & -1.170012 & -2.910916 & -0.513717 & 6 & -0.564998 & 0.649173 & 1.228255 \\
\hline & & & & 6 & -0.716599 & 1.805673 & 2.226589 \\
\hline E ( & 40.6276601 & & & 1 & -0.833148 & 2.768087 & 1.711259 \\
\hline \multirow{2}{*}{\multicolumn{4}{|c|}{ NImag $=1(-356.2)$}} & 1 & -1.612861 & 1.648886 & 2.834629 \\
\hline & & & & 1 & 0.144898 & 1.888634 & 2.902574 \\
\hline \multirow{2}{*}{\multicolumn{4}{|c|}{ 89. TS-5 $t$ conformer $2(-940.4182558)$}} & 1 & -0.492502 & -0.289797 & 1.796440 \\
\hline & & & & 6 & -1.722086 & 0.489188 & 0.271451 \\
\hline 6 & -2.957369 & 1.494439 & 0.765976 & 1 & -2.312651 & 1.366844 & 0.024770 \\
\hline 1 & -2.917428 & 1.144910 & 1.800879 & 6 & -2.231767 & -0.788215 & 0.016346 \\
\hline
\end{tabular}




$\begin{array}{rrrr}6 & -3.488082 & -0.916622 & -0.840276 \\ 1 & -3.909470 & 0.049935 & -1.137400 \\ 1 & -3.258402 & -1.494378 & -1.744010 \\ 1 & -4.248071 & -1.481401 & -0.287092 \\ 8 & -1.709086 & -1.869577 & 0.435582 \\ 6 & 0.324070 & 0.908908 & -1.134820 \\ 8 & 0.666294 & 0.061207 & -1.953101 \\ 1 & 1.673252 & -2.918673 & 0.557150 \\ 1 & 4.031199 & -1.086093 & -0.275736 \\ 6 & -0.159857 & 2.277167 & -1.584980 \\ 1 & -0.600588 & 2.869560 & -0.781165 \\ 1 & 0.702374 & 2.826039 & -1.995193 \\ 1 & -0.890532 & 2.151667 & -2.386871\end{array}$

92. TS-5R (-940.41880)

$\mathrm{E}(\mathrm{MeCN})=-940.6393289$

NImag $=0$

91. I-5 $t$ conformer2 (-940.4454203)

$\begin{array}{ccccc}16 & -1.723922 & -0.976750 & -0.577935 & 6 \\ 6 & -2.879243 & -0.808435 & 0.818549 & 6 \\ 6 & -0.871896 & -2.560308 & -0.229103 & 1 \\ 6 & -0.323569 & 0.233203 & -0.307617 & 1 \\ 6 & 0.403701 & 0.163839 & 1.108665 & 1 \\ 6 & 1.872593 & 0.326597 & 0.877656 & 8 \\ 6 & 2.520889 & -0.496886 & -0.027092 & 6 \\ 8 & 1.922132 & -1.400520 & -0.726434 & 8 \\ 6 & 4.014772 & -0.324931 & -0.268985 & 1 \\ 6 & -0.153231 & 1.155669 & 2.140518 & 1 \\ 1 & -3.341844 & 0.173186 & 0.714538 & 6 \\ 1 & -3.627995 & -1.598442 & 0.722089 & 1 \\ 1 & -2.347393 & -0.896437 & 1.766725 & 1 \\ 1 & -1.307123 & -3.317391 & -0.884385 & 1\end{array}$

$\begin{array}{crrr}6 & 2.868066 & 0.394829 & 1.700098 \\ 1 & 3.056454 & 1.295695 & 1.115041 \\ 1 & 2.242334 & 0.616956 & 2.567205 \\ 16 & 1.966137 & -0.821636 & 0.677990 \\ 6 & 3.083331 & -0.926347 & -0.766580 \\ 1 & 3.110200 & 0.044660 & -1.258287 \\ 1 & 2.691795 & -1.700188 & -1.425386 \\ 6 & 0.379124 & 0.115943 & 0.204185 \\ 1 & -0.049485 & 0.223936 & 1.204744 \\ 6 & -0.725752 & -0.737198 & -0.637078 \\ 6 & -0.131920 & -1.964368 & -1.344697 \\ 1 & 0.252138 & -2.711112 & -0.635482 \\ 1 & -0.936298 & -2.448403 & -1.905273 \\ 1 & 0.660797 & -1.711440 & -2.061518 \\ 1 & -1.049621 & -0.024342 & -1.406099 \\ 6 & -1.916410 & -1.026144 & 0.196746 \\ 1 & -1.950431 & -1.955741 & 0.761530 \\ 6 & -2.999470 & -0.147198 & 0.180581 \\ 6 & -4.253739 & -0.538940 & 0.965208 \\ 1 & -4.161915 & -1.501422 & 1.481769 \\ 1 & -4.486206 & 0.239755 & 1.702784 \\ 1 & -5.108699 & -0.586626 & 0.279561 \\ 8 & -3.043553 & 0.960977 & -0.444573 \\ 6 & 0.670877 & 1.496177 & -0.402829 \\ 8 & 1.723908 & 1.740097 & -0.979513 \\ 1 & 4.060872 & -1.228082 & -0.382216 \\ 1 & 3.801623 & -0.065008 & 2.033223 \\ 6 & -0.448807 & 2.480305 & -0.255790 \\ 1 & -1.437668 & 2.008116 & -0.455956 \\ 1 & -0.485852 & 2.816153 & 0.791034 \\ 1 & -0.262875 & 3.342320 & -0.900885 \\ & & & \end{array}$

$\begin{array}{llll}1 & -1.004452 & -2.838323 & 0.818003\end{array}$

$\begin{array}{lllll}1 & 0.198321 & -2.382780 & -0.442709\end{array}$

$\begin{array}{lllll}1 & 0.420358 & -0.214812 & -0.996286\end{array}$

$\begin{array}{llll}1 & 0.190138 & -0.854634 & 1.473705\end{array}$

$\begin{array}{llll}1 & -1.234710 & 1.063710 & 2.293278\end{array}$

$\begin{array}{lllll}1 & 0.340456 & 0.981257 & 3.102635\end{array}$

$\begin{array}{llll}1 & 0.053518 & 2.193746 & 1.857383\end{array}$

$\begin{array}{llll}1 & 2.402205 & 1.100950 & 1.424616\end{array}$

$\begin{array}{lllll}1 & 4.458163 & 0.467847 & 0.343646\end{array}$

$\begin{array}{lllll}1 & 4.533520 & -1.267563 & -0.054457\end{array}$

$\begin{array}{lllll}1 & 4.196625 & -0.100153 & -1.327582\end{array}$

$\begin{array}{llll}6 & -0.853309 & 1.576959 & -0.725690\end{array}$

$\begin{array}{lllll}8 & -2.059676 & 1.771461 & -0.850796\end{array}$

$\begin{array}{llll}6 & 0.176567 & 2.645282 & -0.997886\end{array}$

$\begin{array}{lllll}1 & -0.275143 & 3.635143 & -0.892926\end{array}$

$\begin{array}{llll}1 & 1.055582 & 2.519516 & -0.355831\end{array}$

$\begin{array}{lllll}1 & 0.529242 & 2.529719 & -2.031739\end{array}$

$\mathrm{E}(\mathrm{MeCN})=-940.6293894$

NImag $=1(-58.9)$

93. TS-5E (-940.43372)

$\mathrm{E}(\mathrm{MeCN})=-940.6441594$

$\begin{array}{lll}3.130941 & -1.073453 & 0.034524\end{array}$

$\begin{array}{llll}2.273470 & 0.536709 & 0.127470\end{array}$

$\begin{array}{llll}0.507385 & 0.257253 & -0.860967\end{array}$

$\begin{array}{llll}-0.466059 & 1.359648 & -0.466350\end{array}$

$\begin{array}{llll}-1.749985 & 0.577241 & -0.570188\end{array}$

$\begin{array}{llll}-2.311937 & 0.102424 & 0.640885\end{array}$

$\begin{array}{lll}-1.735490 & 0.229259 & 1.748959\end{array}$

$\begin{array}{lll}1.660878 & 0.540804 & 1.860548\end{array}$

$\begin{array}{llll}-0.358553 & 2.610939 & -1.341330\end{array}$

$\begin{array}{lll}-3.643958 & -0.639543 & 0.592118\end{array}$

$\begin{array}{llll}2.471300 & -1.849776 & 0.422267\end{array}$

$\begin{array}{llll}3.370841 & -1.259031 & -1.015206\end{array}$

NImag $=0$ 


$\begin{array}{rrrr}1 & 0.657428 & 0.101875 & 1.904048 \\ 1 & 1.657056 & 1.580281 & 2.193190 \\ 1 & 0.823588 & 0.350875 & -1.909802 \\ 1 & -0.545037 & 2.374854 & -2.396982 \\ 1 & -1.103167 & 3.350141 & -1.029236 \\ 1 & 0.631673 & 3.080990 & -1.274222 \\ 1 & -0.354913 & 1.626502 & 0.590590 \\ 1 & -2.298509 & 0.558968 & -1.507239 \\ 1 & -4.103827 & -0.637536 & -0.402138 \\ 1 & -3.489469 & -1.678186 & 0.909064 \\ 1 & -4.339500 & -0.187033 & 1.307766 \\ 1 & 2.371220 & -0.033795 & 2.459412 \\ 1 & 4.056891 & -1.004245 & 0.610859 \\ 6 & -0.014423 & -1.161513 & -0.575556 \\ 8 & 0.138878 & -1.693912 & 0.523241 \\ 6 & -0.406824 & -1.998689 & -1.787805 \\ 1 & -0.739796 & -1.401047 & -2.639384 \\ 1 & 0.453084 & -2.610856 & -2.101698 \\ 1 & -1.206271 & -2.683771 & -1.493691\end{array}$

$\mathrm{E}(\mathrm{MeCN})=-940.6319994$

NImag $=1(-158.6)$

94. TS-5E (syn) (-940.41799)

$\begin{array}{crrr}6 & -0.171135 & 2.408036 & -0.756323 \\ 16 & 0.789300 & 1.507318 & 0.503192 \\ 6 & 0.336789 & -0.680787 & -0.337077 \\ 6 & 1.762824 & -1.087143 & -0.469514 \\ 6 & 2.013149 & -1.997636 & -1.667359 \\ 6 & 2.445638 & 2.213406 & 0.150652 \\ 6 & -0.565056 & -1.373629 & 0.669179 \\ 6 & -1.644445 & -0.335378 & 0.811904 \\ 6 & -2.606528 & -0.235245 & -0.214603 \\ 8 & -2.523904 & -0.925826 & -1.265468 \\ 6 & 0.092696 & -1.812842 & 1.977659 \\ 6 & -3.779646 & 0.730020 & -0.052110 \\ 8 & 2.648287 & -0.807857 & 0.325272 \\ 1 & 0.290998 & 2.304868 & -1.742664 \\ 1 & -1.168925 & 1.967860 & -0.762470 \\ 1 & 2.749744 & 1.993135 & -0.876020 \\ 1 & 3.141544 & 1.737920 & 0.841378 \\ 1 & -0.216212 & -0.516362 & -1.263607 \\ 1 & 0.519903 & -0.963246 & 2.521508 \\ 1 & -0.660938 & -2.280019 & 2.619787 \\ 1 & 0.897524 & -2.536594 & 1.810385 \\ 1 & -0.985610 & -2.247185 & 0.145158 \\ 1 & -1.621585 & 0.336598 & 1.663960 \\ 1 & -3.732072 & 1.313679 & 0.873835 \\ 1 & -3.824822 & 1.415446 & -0.907416 \\ 1 & -4.716196 & 0.160097 & -0.057303 \\ 1 & 2.426858 & 3.295956 & 0.310201\end{array}$

$\begin{array}{rrrr}1 & -0.235886 & 3.464221 & -0.482117 \\ 1 & 1.368421 & -2.884193 & -1.628065 \\ 1 & 1.776960 & -1.476160 & -2.603064 \\ 1 & 3.060715 & -2.306449 & -1.667428\end{array}$

$\mathrm{E}(\mathrm{MeCN})=-940.61593$

NImag $=1(-245.5)$

95. PC-5 (-940.51847)

$\begin{array}{crrr}6 & 3.823650 & -1.279895 & 0.231429 \\ 16 & 3.484519 & 0.239993 & -0.726131 \\ 6 & -0.651610 & 0.295307 & -0.500787 \\ 6 & -1.217623 & 1.385688 & 0.331745 \\ 6 & -2.155653 & 0.567113 & -0.569949 \\ 6 & -3.131698 & -0.356755 & 0.077989 \\ 8 & -2.794956 & -1.137249 & 0.955538 \\ 6 & 3.057095 & 1.362112 & 0.652460 \\ 6 & -0.959957 & 2.845591 & 0.032993 \\ 6 & -4.570471 & -0.262235 & -0.396546 \\ 1 & 2.945252 & -1.565441 & 0.817025 \\ 1 & 4.055696 & -2.070832 & -0.487108 \\ 1 & 2.189893 & 0.979382 & 1.198319 \\ 1 & 2.814295 & 2.334327 & 0.214181 \\ 1 & -0.115615 & 0.578549 & -1.407345 \\ 1 & -0.879364 & 3.024757 & -1.045611 \\ 1 & -1.764056 & 3.481345 & 0.421829 \\ 1 & -0.021125 & 3.171663 & 0.496057 \\ 1 & -1.302923 & 1.127030 & 1.385819 \\ 1 & -2.509640 & 1.074252 & -1.465251 \\ 1 & -4.943809 & 0.763969 & -0.289733 \\ 1 & -4.636728 & -0.515880 & -1.462477 \\ 1 & -5.196855 & -0.944804 & 0.180762 \\ 1 & 3.905380 & 1.486796 & 1.332949 \\ 1 & 4.685079 & -1.141799 & 0.892464 \\ 6 & -0.060885 & -0.949242 & 0.139799 \\ 8 & 0.508381 & -0.892189 & 1.214638 \\ 6 & -0.151574 & -2.231085 & -0.659819 \\ 1 & -1.180368 & -2.605685 & -0.604185 \\ 1 & 0.086024 & -2.059405 & -1.715909 \\ 1 & 0.521526 & -2.982216 & -0.240975\end{array}$

$\mathrm{E}(\mathrm{MeCN})=-940.7016938$

NImag $=0$

96. TS-5'c (-940.43737)

$\begin{array}{cccc}6 & -1.879637 & -2.272575 & -0.894616 \\ 1 & -2.720342 & -1.936095 & -0.287524 \\ 1 & -2.037139 & -2.034358 & -1.948870 \\ 16 & -0.332734 & -1.429033 & -0.366079 \\ 6 & -0.359797 & -1.882551 & 1.403504\end{array}$




\begin{tabular}{|c|c|c|c|c|c|c|c|}
\hline 1 & -1.237654 & -1.436170 & 1.868375 & 1 & 4.578763 & -0.529876 & -0.875358 \\
\hline 1 & 0.591110 & -1.519552 & 1.791959 & 1 & 4.046644 & 1.147666 & -0.852188 \\
\hline 6 & -0.748579 & 0.333001 & -0.479627 & 8 & 1.995945 & -0.244669 & -1.782003 \\
\hline 1 & -0.509511 & 0.626479 & -1.499634 & 6 & -2.085725 & -0.562787 & -0.520413 \\
\hline 6 & 0.648398 & 1.312938 & 0.461488 & 8 & -2.137788 & 0.001252 & -1.619076 \\
\hline 6 & 0.084787 & 2.715116 & 0.624226 & 1 & -1.244553 & 3.532254 & -0.255606 \\
\hline 1 & 0.767332 & 3.288396 & 1.262741 & 1 & 0.698327 & 2.896068 & 1.621887 \\
\hline 1 & 0.020409 & 3.243391 & -0.334160 & 6 & -3.016332 & -1.725726 & -0.210389 \\
\hline 1 & -0.901579 & 2.726920 & 1.098354 & 1 & -3.166559 & -1.886370 & 0.861570 \\
\hline 6 & 1.897273 & 1.196880 & -0.210478 & 1 & -3.978141 & -1.545177 & -0.698319 \\
\hline 1 & 2.215042 & 1.994954 & -0.877660 & 1 & -2.598025 & -2.646193 & -0.637375 \\
\hline 6 & 2.696116 & 0.045751 & -0.053254 & 1 & 0.292838 & -1.700984 & -0.719414 \\
\hline 6 & 4.087461 & 0.019270 & -0.678033 & & & & \\
\hline 1 & 4.333970 & 0.938881 & -1.218689 & \multicolumn{4}{|c|}{$\mathrm{E}(\mathrm{MeCN})=-940.629293$} \\
\hline 1 & 4.834314 & -0.138589 & 0.109184 & \multicolumn{4}{|c|}{ NImag = $1(-340.6)$} \\
\hline 1 & 4.167686 & -0.832003 & -1.364957 & & & & \\
\hline 8 & 2.320302 & -0.978791 & 0.583301 & \multicolumn{4}{|c|}{ 98. I-5'c conformer1 (-940.4460942) } \\
\hline 6 & -2.105133 & 0.694770 & -0.031068 & & & & \\
\hline 8 & -2.672836 & 0.149674 & 0.920991 & 6 & -1.842769 & -2.302939 & -0.779661 \\
\hline 1 & -0.388617 & -2.974855 & 1.437669 & 1 & -2.477872 & -2.149932 & 0.094181 \\
\hline 1 & -1.720032 & -3.348753 & -0.785499 & 1 & -2.356131 & -2.000224 & -1.696677 \\
\hline 6 & -2.799129 & 1.821279 & -0.787223 & 16 & -0.248123 & -1.329950 & -0.658999 \\
\hline 1 & -2.104048 & 2.482333 & -1.311136 & 6 & 0.280688 & -1.846839 & 1.009234 \\
\hline 1 & -3.469259 & 1.376666 & -1.535713 & 1 & -0.571000 & -1.775606 & 1.684530 \\
\hline 1 & -3.415083 & 2.401391 & -0.094705 & 1 & 1.118688 & -1.198276 & 1.330132 \\
\hline \multirow[t]{2}{*}{1} & 0.586861 & 0.735162 & 1.383686 & 6 & -0.924286 & 0.391204 & -0.490125 \\
\hline & & & & 1 & -1.132230 & 0.661200 & -1.533879 \\
\hline \multicolumn{4}{|c|}{$E(\mathrm{MeCN})=-940.6341621$} & 6 & 0.310601 & 1.226781 & 0.017022 \\
\hline \multirow{2}{*}{\multicolumn{4}{|c|}{ NImag $=1(-323.3)$}} & 6 & 0.090486 & 2.730758 & -0.218910 \\
\hline & & & & 1 & 0.970838 & 3.272092 & 0.138988 \\
\hline \multirow{2}{*}{\multicolumn{4}{|c|}{ 97. TS-5'c conformer2 (-940.4258691) }} & 1 & -0.024235 & 2.955435 & -1.287722 \\
\hline & & & & 1 & -0.783755 & 3.129089 & 0.313154 \\
\hline 6 & 0.264801 & 1.898428 & 1.727490 & 6 & 1.548520 & 0.676709 & -0.624760 \\
\hline 1 & -0.572850 & 1.916457 & 2.430364 & 1 & 1.706737 & 0.896289 & -1.679923 \\
\hline 1 & 1.033540 & 1.190453 & 2.034786 & 6 & 2.627786 & 0.235623 & 0.161692 \\
\hline 16 & -0.290991 & 1.360124 & 0.076881 & 6 & 3.956594 & -0.057295 & -0.536417 \\
\hline 6 & -1.680158 & 2.537813 & -0.125906 & 1 & 3.917622 & 0.080595 & -1.622438 \\
\hline 1 & -2.341651 & 2.519168 & 0.742955 & 1 & 4.730010 & 0.603549 & -0.126174 \\
\hline 1 & -2.209303 & 2.230082 & -1.026576 & 1 & 4.271215 & -1.084337 & -0.315351 \\
\hline 6 & -1.064566 & -0.229510 & 0.471858 & 8 & 2.563568 & 0.017832 & 1.407373 \\
\hline 1 & -1.350539 & -0.278807 & 1.522566 & 6 & -2.182934 & 0.527688 & 0.358541 \\
\hline 6 & 0.463517 & -1.438069 & 0.325269 & 8 & -2.338322 & -0.103749 & 1.390767 \\
\hline 6 & 0.097395 & -2.525525 & 1.320616 & 1 & 0.621769 & -2.879763 & 0.897624 \\
\hline 1 & 0.756257 & -3.388384 & 1.164689 & 1 & -1.551036 & -3.352483 & -0.864735 \\
\hline 1 & 0.246729 & -2.185907 & 2.353173 & 6 & -3.227916 & 1.509672 & -0.137654 \\
\hline 1 & -0.936268 & -2.873196 & 1.222462 & 1 & -2.780094 & 2.416344 & -0.555000 \\
\hline 6 & 1.712590 & -0.775269 & 0.505330 & 1 & -3.804480 & 1.033789 & -0.943513 \\
\hline 1 & 2.175980 & -0.778823 & 1.491608 & 1 & -3.909719 & 1.763547 & 0.676791 \\
\hline 6 & 2.449659 & -0.292448 & -0.623937 & 1 & 0.401045 & 1.047061 & 1.095104 \\
\hline 6 & 3.891838 & 0.169429 & -0.383257 & & & & \\
\hline 1 & 4.161303 & 0.228914 & 0.677345 & \multicolumn{4}{|c|}{$\begin{array}{l}\mathrm{E}(\mathrm{MeCN})=-940.6460102 \\
\text { NImag }=0\end{array}$} \\
\hline
\end{tabular}


99. I-5' $c$ conformer2 (-940.4463573)

$\begin{array}{crrr}6 & -1.515515 & -2.084979 & -1.199353 \\ 1 & -2.419572 & -1.972252 & -0.594822 \\ 1 & -1.645210 & -1.606288 & -2.173730 \\ 16 & 0.014079 & -1.359271 & -0.388513 \\ 6 & -0.262856 & -2.151022 & 1.241780 \\ 1 & -0.997077 & -1.568680 & 1.798768 \\ 1 & 0.708435 & -2.152494 & 1.728427 \\ 6 & -0.633129 & 0.412723 & -0.377240 \\ 1 & -0.668233 & 0.617342 & -1.452549 \\ 6 & 0.372042 & 1.421415 & 0.280980 \\ 6 & -0.194804 & 2.848567 & 0.191099 \\ 1 & 0.525784 & 3.539194 & 0.640455 \\ 1 & -0.339848 & 3.161215 & -0.851611 \\ 1 & -1.144894 & 2.967329 & 0.724667 \\ 6 & 1.744553 & 1.326531 & -0.315984 \\ 1 & 2.132841 & 2.150366 & -0.907513 \\ 6 & 2.489391 & 0.228240 & 0.004772 \\ 6 & 3.938559 & 0.072854 & -0.408332 \\ 1 & 4.310435 & 0.942028 & -0.961436 \\ 1 & 4.563975 & -0.069404 & 0.481961 \\ 1 & 4.064193 & -0.823979 & -1.028499 \\ 8 & 1.957512 & -0.753050 & 0.682075 \\ 6 & -2.034008 & 0.523077 & 0.226935 \\ 8 & -2.264853 & 0.187815 & 1.376784 \\ 1 & -0.633662 & -3.161205 & 1.051626 \\ 1 & -1.312126 & -3.147755 & -1.357752 \\ 6 & -3.106695 & 1.137915 & -0.652777 \\ 1 & -2.766144 & 2.089625 & -1.076055 \\ 1 & -3.326520 & 0.473386 & -1.498353 \\ 1 & -4.015710 & 1.294847 & -0.068356 \\ 1 & 0.411561 & 1.125013 & 1.338837 \\ 1 & & & \\ 1 & & & \\ 1 & & & \\ 1 & & & \end{array}$

$\mathrm{E}(\mathrm{MeCN})=-940.6425854$

$\mathrm{NImag}=0$

100.I-5'c conformer3 (-940.4434042)

$\begin{array}{crrr}6 & 0.962826 & -1.985901 & -1.009359 \\ 1 & 0.397858 & -2.578504 & -1.731969 \\ 1 & 1.394625 & -1.096332 & -1.474709 \\ 16 & -0.133917 & -1.411201 & 0.336889 \\ 6 & -1.626846 & -2.401993 & -0.205972 \\ 1 & -1.937153 & -2.174767 & -1.230716 \\ 1 & -2.438343 & -2.205743 & 0.495558 \\ 6 & -0.739832 & 0.256019 & -0.306044 \\ 1 & -0.734347 & 0.173389 & -1.398176 \\ 6 & 0.193441 & 1.440196 & 0.150866 \\ 6 & -0.441259 & 2.787015 & -0.237502 \\ 1 & 0.230480 & 3.589738 & 0.082721 \\ 1 & -0.562825 & 2.878487 & -1.325002\end{array}$

$\begin{array}{rrr}-1.413311 & 2.960371 & 0.237446 \\ 1.602066 & 1.344912 & -0.359852 \\ 1.974003 & 2.120478 & -1.023235 \\ 2.427943 & 0.402668 & 0.194795 \\ 3.920507 & 0.383867 & -0.085859 \\ 4.233566 & 1.190927 & -0.756819 \\ 4.470139 & 0.480039 & 0.858574 \\ 4.217158 & -0.578310 & -0.523806 \\ 1.968088 & -0.532152 & 0.970366 \\ -2.153124 & 0.454034 & 0.234488 \\ -2.401686 & 0.262591 & 1.412114 \\ -1.338711 & -3.454587 & -0.134523 \\ 1.754542 & -2.562628 & -0.534680 \\ -3.212097 & 0.916905 & -0.748704 \\ -2.845088 & 1.716761 & -1.399376 \\ -3.493529 & 0.076934 & -1.398648 \\ -4.096370 & 1.251657 & -0.202077 \\ 0.197010 & 1.361476 & 1.248232\end{array}$

$\mathrm{E}(\mathrm{MeCN})=-940.6415894$

NImag $=0$

101. TS-5't (-940.42505)

$\begin{array}{rrr}-2.735768 & -1.387851 & -1.206562 \\ -3.082823 & -0.401967 & -1.517217 \\ -2.153916 & -1.869821 & -1.994769 \\ -1.614867 & -1.192935 & 0.229393 \\ -2.740200 & -0.345226 & 1.391009 \\ -3.029649 & 0.611835 & 0.958669 \\ -2.202731 & -0.215282 & 2.330901 \\ -0.353913 & -0.006275 & -0.259732 \\ 0.391594 & -0.630564 & -0.769432 \\ 0.872748 & 0.305440 & 1.205904 \\ 0.368346 & 1.468372 & 2.042978 \\ 0.919376 & 1.496419 & 2.991213 \\ 0.540446 & 2.424910 & 1.537606 \\ -0.698540 & 1.407896 & 2.277243 \\ 2.190600 & 0.416428 & 0.671762 \\ 2.713153 & 1.368478 & 0.725095 \\ 2.740481 & -0.671503 & -0.044908 \\ 4.180904 & -0.590699 & -0.541382 \\ 4.675588 & 0.348111 & -0.270528 \\ 4.754949 & -1.424451 & -0.119637 \\ 4.206697 & -0.708307 & -1.631519 \\ 2.067271 & -1.701838 & -0.321358 \\ -0.840786 & 1.217512 & -0.903961 \\ -2.013055 & 1.616132 & -0.836126 \\ -3.597020 & -1.004845 & 1.549562 \\ -3.563493 & -2.037079 & -0.909035 \\ 0.218733 & 2.011277 & -1.639759 \\ 1.170415 & 1.995372 & -1.092344\end{array}$




\begin{tabular}{|c|c|c|c|c|c|c|c|}
\hline 1 & 0.415259 & 1.549071 & -2.615898 & 8 & -2.099208 & 1.804402 & -0.568861 \\
\hline 1 & -0.133913 & 3.033693 & -1.797654 & 6 & 0.117841 & 2.519553 & -1.149804 \\
\hline \multirow[t]{2}{*}{1} & 0.671886 & -0.675721 & 1.645242 & 6 & 2.026272 & 0.372957 & 0.606798 \\
\hline & & & & 6 & 2.415638 & -0.630658 & -0.266202 \\
\hline \multicolumn{4}{|c|}{$\mathrm{E}(\mathrm{MeCN})=-940.6300613$} & 6 & 3.814406 & -0.607981 & -0.868513 \\
\hline \multirow{2}{*}{\multicolumn{4}{|c|}{ NImag $=1(-356.2)$}} & 8 & 1.638775 & -1.590556 & -0.640861 \\
\hline & & & & 1 & -0.064022 & -2.380993 & 0.261088 \\
\hline \multirow{2}{*}{\multicolumn{4}{|c|}{ 102.TS-5't conformer 2(-940.4389337) }} & 1 & -0.963670 & -2.489445 & 1.824242 \\
\hline & & & & 1 & -1.768624 & -1.488512 & -1.905737 \\
\hline 6 & -0.712188 & -2.555343 & 0.167888 & 1 & -3.000589 & -0.205150 & -1.615226 \\
\hline 1 & -0.868477 & -2.895529 & -0.858650 & 1 & 0.158722 & -0.381440 & -0.765564 \\
\hline 1 & 0.352394 & -2.311910 & 0.302197 & 1 & 1.006472 & 1.398367 & 3.029908 \\
\hline 16 & -1.666567 & -1.023646 & 0.450296 & 1 & 0.486639 & 2.459789 & 1.721526 \\
\hline 6 & -3.111103 & -1.296756 & -0.649059 & 1 & -0.698199 & 1.426738 & 2.540591 \\
\hline 1 & -2.767584 & -1.552744 & -1.653796 & 1 & 2.694332 & 1.182972 & 0.883541 \\
\hline 1 & -3.661259 & -0.355638 & -0.649570 & 1 & 4.416334 & 0.238181 & -0.519155 \\
\hline 6 & -0.586543 & 0.186385 & -0.303617 & 1 & 4.338064 & -1.539219 & -0.618990 \\
\hline 1 & 0.055814 & -0.257508 & -1.058192 & 1 & 3.747943 & -0.568849 & -1.963304 \\
\hline 6 & 0.904137 & 0.554107 & 1.084747 & 1 & -3.339450 & -1.899254 & -1.134908 \\
\hline 6 & 0.372862 & 1.678863 & 1.944937 & 1 & -1.706104 & -3.177739 & 0.357555 \\
\hline 1 & 0.926371 & 1.696068 & 2.892771 & 1 & 1.081773 & 2.444697 & -0.636491 \\
\hline 1 & 0.512501 & 2.654662 & 1.467234 & 1 & 0.303195 & 2.237935 & -2.195156 \\
\hline 1 & -0.690171 & 1.565131 & 2.178780 & 1 & -0.286257 & 3.535303 & -1.131312 \\
\hline 6 & 2.119207 & 0.693001 & 0.396159 & 1 & 0.500959 & -0.611520 & 1.722327 \\
\hline 1 & 2.548849 & 1.679884 & 0.242709 & & & & \\
\hline 6 & 2.737670 & -0.451526 & -0.182099 & \multicolumn{4}{|c|}{$\mathrm{E}(\mathrm{MeCN})=-940.645224$} \\
\hline 6 & 4.099725 & -0.292912 & -0.849788 & \multicolumn{4}{|c|}{ NImag $=0$} \\
\hline 1 & 4.469758 & 0.737118 & -0.830977 & & & & \\
\hline 1 & 4.827263 & -0.938690 & -0.343972 & \multicolumn{4}{|c|}{ 104. I-5't conformer2 (-940.4454203) } \\
\hline 1 & 4.042850 & -0.634207 & -1.890260 & & & & \\
\hline 8 & 2.214074 & -1.593947 & -0.181402 & 16 & -1.723922 & -0.976750 & -0.577935 \\
\hline 6 & -1.296502 & 1.419393 & -0.563199 & 6 & -2.879243 & -0.808435 & 0.818549 \\
\hline 8 & -2.415439 & 1.651018 & -0.074953 & 6 & -0.871896 & -2.560308 & -0.229103 \\
\hline 1 & -3.714078 & -2.103805 & -0.224465 & 6 & -0.323569 & 0.233203 & -0.307617 \\
\hline 1 & -1.057067 & -3.309223 & 0.878117 & 6 & 0.403701 & 0.163839 & 1.108665 \\
\hline 6 & -0.571577 & 2.446644 & -1.414030 & 6 & 1.872593 & 0.326597 & 0.877656 \\
\hline 1 & 0.510390 & 2.420446 & -1.238940 & 6 & 2.520889 & -0.496886 & -0.027092 \\
\hline 1 & -0.735078 & 2.229717 & -2.477699 & 8 & 1.922132 & -1.400520 & -0.726434 \\
\hline 1 & -0.971570 & 3.441232 & -1.200878 & 6 & 4.014772 & -0.324931 & -0.268985 \\
\hline \multirow[t]{2}{*}{1} & 0.760966 & -0.434371 & 1.520056 & 6 & -0.153231 & 1.155669 & 2.140518 \\
\hline & & & & 1 & -3.341844 & 0.173186 & 0.714538 \\
\hline \multicolumn{4}{|c|}{$\mathrm{E}(\mathrm{MeCN})=-940.6346309$} & 1 & -3.627995 & -1.598442 & 0.722089 \\
\hline \multirow{2}{*}{\multicolumn{4}{|c|}{ NImag $=1(-314.2)$}} & 1 & -2.347393 & -0.896437 & 1.766725 \\
\hline & & & & 1 & -1.307123 & -3.317391 & -0.884385 \\
\hline \multirow{2}{*}{\multicolumn{4}{|c|}{ 103. I-5't conformer1 (-940.4477043) }} & 1 & -1.004452 & -2.838323 & 0.818003 \\
\hline & & & & 1 & 0.198321 & -2.382780 & -0.442709 \\
\hline 6 & -1.055161 & -2.388664 & 0.741925 & 1 & 0.420358 & -0.214812 & -0.996286 \\
\hline 16 & -1.878303 & -0.779845 & 0.401808 & 1 & 0.190138 & -0.854634 & 1.473705 \\
\hline 6 & -2.567356 & -1.134916 & -1.248805 & 1 & -1.234710 & 1.063710 & 2.293278 \\
\hline 6 & -0.366728 & 0.262087 & -0.033586 & 1 & 0.340456 & 0.981257 & 3.102635 \\
\hline 6 & 0.647092 & 0.338922 & 1.184444 & 1 & 0.053518 & 2.193746 & 1.857383 \\
\hline 6 & 0.333261 & 1.471293 & 2.169016 & 1 & 2.402205 & 1.100950 & 1.424616 \\
\hline 6 & -0.893800 & 1.561556 & -0.569624 & 1 & 4.458163 & 0.467847 & 0.343646 \\
\hline
\end{tabular}




\begin{tabular}{|c|c|c|c|c|c|c|c|}
\hline 1 & 4.533520 & -1.267563 & -0.054457 & 6 & -0.227358 & 0.321272 & -0.000015 \\
\hline 1 & 4.196625 & -0.100153 & -1.327582 & 6 & -0.633785 & 1.675820 & -0.530838 \\
\hline 6 & -0.853309 & 1.576959 & -0.725690 & 6 & -1.459894 & -2.272702 & 0.733913 \\
\hline 8 & -2.059676 & 1.771461 & -0.850796 & 6 & 0.693319 & 0.172885 & 1.219312 \\
\hline 6 & 0.176567 & 2.645282 & -0.997886 & 6 & 2.014652 & 0.252135 & 0.525505 \\
\hline 1 & -0.275143 & 3.635143 & -0.892926 & 6 & 2.385903 & -0.817700 & -0.303927 \\
\hline 1 & 1.055582 & 2.519516 & -0.355831 & 6 & 3.738519 & -0.783902 & -1.006756 \\
\hline 1 & 0.529242 & 2.529719 & -2.031739 & 6 & 0.451472 & 1.164789 & 2.357829 \\
\hline & & & & 8 & 1.630402 & -1.817757 & -0.519101 \\
\hline E (I & 940.644159 & & & 8 & -1.812446 & 1.916019 & -0.772575 \\
\hline NIn & & & & 1 & -2.995225 & 0.022947 & -1.788776 \\
\hline & & & & 1 & -3.418277 & -1.674379 & -1.387760 \\
\hline 105 & 940.43714) & & & 1 & -1.772436 & -1.277412 & -1.975374 \\
\hline & & & & 1 & -2.190793 & -3.017708 & 0.409197 \\
\hline 6 & 0.805181 & -2.548784 & 0.648169 & 1 & -0.488734 & -2.416314 & 0.245128 \\
\hline 1 & 1.297517 & -2.363601 & 1.606392 & 1 & -1.347738 & -2.330897 & 1.818063 \\
\hline 1 & -0.290329 & -2.488645 & 0.710026 & 1 & 0.092708 & -0.408767 & -0.751081 \\
\hline 16 & 1.257960 & -1.253983 & -0.551531 & 1 & -0.573105 & 1.098152 & 2.744920 \\
\hline 6 & 3.056349 & -1.118144 & -0.238266 & 1 & 1.139553 & 0.949198 & 3.182086 \\
\hline 1 & 3.262968 & -0.902046 & 0.809886 & 1 & 0.623875 & 2.203718 & 2.055449 \\
\hline 1 & 3.450320 & -0.315785 & -0.858250 & 1 & 2.645084 & 1.125947 & 0.653526 \\
\hline 6 & 0.346552 & 0.250698 & 0.132256 & 1 & 4.321176 & -1.667142 & -0.719009 \\
\hline 1 & -0.282790 & -0.227147 & 0.879866 & 1 & 3.594057 & -0.841525 & -2.092800 \\
\hline 6 & -0.668570 & 0.781940 & -1.013538 & 1 & 4.321687 & 0.113487 & -0.772952 \\
\hline 6 & -0.227930 & 2.102431 & -1.656583 & 1 & 0.550660 & -0.845397 & 1.603508 \\
\hline 1 & -0.892803 & 2.320059 & -2.498982 & 6 & 0.428513 & 2.711247 & -0.831886 \\
\hline 1 & -0.298237 & 2.949019 & -0.963590 & 1 & 1.152120 & 2.807980 & -0.020085 \\
\hline 1 & 0.799384 & 2.063438 & -2.037222 & 1 & 0.997644 & 2.398757 & -1.714972 \\
\hline 6 & -2.039360 & 0.790611 & -0.434159 & 1 & -0.063622 & 3.666087 & -1.035022 \\
\hline 1 & -2.624265 & 1.705001 & -0.421172 & & & & \\
\hline 6 & -2.529313 & -0.426580 & 0.006783 & \multicolumn{4}{|c|}{$\mathrm{E}(\mathrm{MeCN})=-940.639339$} \\
\hline 6 & -3.936051 & -0.536081 & 0.577611 & \multicolumn{4}{|c|}{ NImag = $1(-190.1)$} \\
\hline 1 & -4.466563 & 0.422491 & 0.584694 & & & & \\
\hline 1 & -4.518414 & -1.256058 & -0.010958 & \multicolumn{4}{|c|}{ 107. TS-5'E (syn) (-940.42497) } \\
\hline 1 & -3.897493 & -0.926596 & 1.602965 & & & & \\
\hline 8 & -1.817919 & -1.501241 & -0.030382 & 6 & -1.818480 & -2.038890 & -1.122721 \\
\hline 6 & 1.212442 & 1.341524 & 0.713688 & 1 & -2.655605 & -1.660119 & -0.530327 \\
\hline 8 & 2.371758 & 1.549039 & 0.385373 & 1 & -1.848366 & -1.601862 & -2.124233 \\
\hline 1 & 3.480839 & -2.082733 & -0.530501 & 16 & -0.197553 & -1.640716 & -0.365430 \\
\hline 1 & 1.126236 & -3.506299 & 0.231256 & 6 & -0.539928 & -2.267397 & 1.308378 \\
\hline 6 & 0.480343 & 2.208814 & 1.717716 & 1 & -1.191404 & -1.557117 & 1.827257 \\
\hline 1 & -0.573151 & 2.328893 & 1.437048 & 1 & 0.425284 & -2.318970 & 1.808285 \\
\hline 1 & 0.490504 & 1.708608 & 2.695728 & 6 & -0.556513 & 0.922176 & -0.476528 \\
\hline 1 & 0.983756 & 3.174258 & 1.810929 & 1 & -0.567870 & 0.979192 & -1.562733 \\
\hline \multirow[t]{2}{*}{1} & -0.603403 & -0.000087 & -1.785606 & 6 & 0.566182 & 1.641331 & 0.217070 \\
\hline & & & & 6 & 0.377079 & 3.166705 & 0.130034 \\
\hline \multicolumn{4}{|c|}{$E(\mathrm{MeCN})=-940.63698$} & 1 & 1.228502 & 3.657472 & 0.618031 \\
\hline \multirow{2}{*}{\multicolumn{4}{|c|}{ NImag $=1(-51.4)$}} & 1 & 0.347900 & 3.506477 & -0.912609 \\
\hline & & & & 1 & -0.543994 & 3.491845 & 0.626549 \\
\hline \multirow{2}{*}{\multicolumn{4}{|c|}{ 106. TS-5'E (-940.44501) }} & 6 & 1.851202 & 1.169260 & -0.376312 \\
\hline & & & & 1 & 2.297025 & 1.746145 & -1.184314 \\
\hline 6 & -2.626455 & -0.921372 & -1.391166 & 6 & 2.436029 & -0.033671 & 0.036530 \\
\hline 16 & -2.107600 & -0.606728 & 0.327971 & 6 & 3.754063 & -0.488547 & -0.574375 \\
\hline
\end{tabular}




$\begin{array}{rrrr}1 & 4.201376 & 0.263906 & -1.232267 \\ 1 & 4.463641 & -0.726522 & 0.226013 \\ 1 & 3.599541 & -1.410247 & -1.149003 \\ 8 & 1.879576 & -0.775394 & 0.900736 \\ 6 & -1.859186 & 0.835514 & 0.203254 \\ 8 & -1.962884 & 0.687497 & 1.419328 \\ 1 & -1.012736 & -3.252338 & 1.245862 \\ 1 & -1.908328 & -3.125771 & -1.214384 \\ 6 & -3.094335 & 1.102668 & -0.654244 \\ 1 & -3.241347 & 2.187380 & -0.740449 \\ 1 & -3.002826 & 0.704050 & -1.669049 \\ 1 & -3.976345 & 0.684182 & -0.162274 \\ 1 & 0.516182 & 1.332046 & 1.269495\end{array}$

$\mathrm{E}(\mathrm{MeCN})=-940.6201654$

NImag $=1(-174.5)$

108. PC-5' (-940.51883)

\begin{tabular}{|c|c|c|c|c|c|c|c|}
\hline 16 & -3.575887 & -0892910 & 0.485061 & \multicolumn{4}{|c|}{$\begin{array}{l}\mathrm{E}(\mathrm{MeCN})=-517.2942184 \\
\mathrm{NImag}=0\end{array}$} \\
\hline 6 & -4.092574 & 0.805064 & 0.046194 & \multirow{2}{*}{\multicolumn{4}{|c|}{$110.2(-748.3077447)$}} \\
\hline 6 & -2.992112 & -1.453644 & -1.154778 & & & & \\
\hline 6 & 0.955843 & 0.154162 & -0.445400 & & & & \\
\hline 6 & 1.064623 & -0.689226 & 0.807285 & 6 & -2.447348 & 0.665624 & -1.387839 \\
\hline 6 & 0.341923 & -0.394761 & 2.104517 & 1 & -1.711014 & 1.470469 & -1.376741 \\
\hline 6 & 2.267020 & 0.110135 & 0.344943 & 1 & -2.357494 & 0.091840 & -2.311905 \\
\hline 6 & 3.411961 & -0.627424 & -0.279301 & 1 & -3.459370 & 1.069606 & -1.291684 \\
\hline 8 & 3.225255 & -1.618569 & -0.965731 & 16 & -2.158190 & -0.544221 & 0.000029 \\
\hline 6 & 4.802586 & -0.085228 & -0.009923 & 6 & -2.446529 & 0.665084 & 1.388368 \\
\hline 1 & -4.926011 & 0.789920 & -0.663357 & 1 & -1.709473 & 1.469267 & 1.377727 \\
\hline 1 & -3.254072 & 1.362961 & -0.379865 & 1 & -3.458172 & 1.070036 & 1.292285 \\
\hline 1 & -4.424906 & 1.291519 & 0.967487 & 1 & -2.357306 & 0.090827 & 2.312195 \\
\hline 1 & -3.817938 & -1.491440 & -1.872381 & 6 & -0.613716 & -1.176515 & -0.000302 \\
\hline 1 & -2.205103 & -0.790667 & -1.524492 & 1 & -0.645889 & -2.259297 & -0.000777 \\
\hline 1 & -2.590835 & -2.462882 & -1.027708 & 6 & 0.664807 & -0.501205 & -0.000233 \\
\hline 1 & 0.964155 & -0.431097 & -1.361455 & 6 & 1.843202 & -1.289150 & -0.000121 \\
\hline 1 & -0.716243 & -0.668951 & 2.022754 & 6 & 3.108442 & -0.714767 & 0.000047 \\
\hline 1 & 0.785229 & -0.976296 & 2.921077 & 6 & 3.264457 & 0.675298 & 0.000117 \\
\hline 1 & 0.394293 & 0.660448 & 2.389162 & 6 & 2.119562 & 1.472679 & -0.000033 \\
\hline 1 & 2.520308 & 0.990818 & 0.929854 & 6 & 0.848083 & 0.901839 & -0.000226 \\
\hline 1 & 4.999907 & -0.058277 & 1.069412 & 1 & 1.746458 & -2.372739 & -0.000141 \\
\hline 1 & 5.547987 & -0.710937 & -0.504268 & 1 & 3.984493 & -1.359742 & 0.000125 \\
\hline 1 & 4.887193 & 0.946430 & -0.375247 & 1 & 4.253796 & 1.123738 & 0.000271 \\
\hline 6 & 0.083883 & 1.369592 & -0.564342 & 1 & 2.212424 & 2.556710 & -0.000025 \\
\hline 8 & -0.876534 & 1.330802 & -1.319247 & 1 & -0.013176 & 1.563511 & -0.000467 \\
\hline 1 & 1.148029 & -1.748591 & 0.571693 & \multirow{5}{*}{\multicolumn{4}{|c|}{$\begin{array}{l}\mathrm{E}(\mathrm{MeCN})=-748.41536 \\
\mathrm{NImag}=0\end{array}$}} \\
\hline 6 & 0.419754 & 2.643001 & 0.190859 & & & & \\
\hline 1 & 1.381545 & 3.039192 & -0.158915 & & & & \\
\hline 1 & -0.362290 & 3.381922 & 0.006432 & & & & \\
\hline 1 & 0.515730 & 2.474610 & 1.268345 & & & & \\
\hline
\end{tabular}


$111.3(-745.1531765)$

$\begin{array}{crrr}6 & -1.939269 & 0.573007 & -1.383658 \\ 1 & -1.166990 & 1.341840 & -1.331522 \\ 1 & -1.855694 & -0.000669 & -2.308454 \\ 1 & -2.942690 & 0.997459 & -1.290896 \\ 16 & -1.653750 & -0.611392 & -0.001303 \\ 6 & -1.939347 & 0.566995 & 1.386346 \\ 1 & -1.167262 & 1.336222 & 1.337644 \\ 1 & -2.942947 & 0.991511 & 1.295920 \\ 1 & -1.855156 & -0.010930 & 2.308465 \\ 6 & -0.026282 & -1.064903 & -0.002669 \\ 1 & 0.160754 & -2.128501 & -0.003673 \\ 6 & 1.002114 & -0.080222 & -0.000440 \\ 8 & 0.847521 & 1.147736 & 0.002116 \\ 8 & 2.250932 & -0.644043 & -0.001365 \\ 6 & 3.333861 & 0.287044 & 0.000927 \\ 1 & 3.304045 & 0.927979 & 0.887677 \\ 1 & 4.242011 & -0.319200 & 0.004532 \\ 1 & 3.309846 & 0.925496 & -0.887889\end{array}$

$\mathrm{E}(\mathrm{MeCN})=-745.2723595$

$\mathrm{NImag}=0$

112. 4 (-861.6695806)

\begin{tabular}{crrr}
6 & 3.183440 & 0.033572 & 1.436388 \\
1 & 2.551981 & 0.908138 & 1.594963 \\
1 & 2.998034 & -0.728581 & 2.195208 \\
1 & 4.245424 & 0.291958 & 1.410819 \\
16 & 2.713939 & -0.707318 & -0.180040 \\
6 & 3.189845 & 0.707165 & -1.260478 \\
1 & 2.558471 & 1.554805 & -0.993042 \\
1 & 4.252083 & 0.920938 & -1.114282 \\
1 & 3.007156 & 0.389330 & -2.288558 \\
6 & 1.016278 & -0.864616 & -0.207874 \\
1 & 0.682179 & -1.844816 & -0.513712 \\
6 & 0.180239 & 0.250363 & 0.065251 \\
8 & 0.627719 & 1.392056 & 0.329541 \\
6 & -1.317899 & 0.054929 & 0.026052 \\
6 & -2.118076 & 1.206367 & 0.024053 \\
6 & -3.507150 & 1.109405 & -0.015389 \\
6 & -4.121244 & -0.145040 & -0.044161 \\
6 & -3.335330 & -1.299135 & -0.029623 \\
6 & -1.944367 & -1.199891 & 0.004508 \\
1 & -1.619312 & 2.169250 & 0.057234 \\
1 & -4.113010 & 2.012154 & -0.021400 \\
1 & -5.205138 & -0.223525 & -0.072288 \\
1 & -3.806008 & -2.279042 & -0.040105 \\
1 & -1.351047 & -2.108682 & 0.037114 \\
& & & \\
\hline & & &
\end{tabular}

$\mathrm{E}(\mathrm{MeCN})=-861.8124342$

NImag $=0$
113. $5(-669.9251225)$

$\begin{array}{lrrr}6 & 1.582488 & -1.384859 & 0.468426 \\ 1 & 0.882564 & -1.324107 & 1.302356 \\ 1 & 1.445298 & -2.310137 & -0.093957 \\ 1 & 2.621594 & -1.291703 & 0.795489 \\ 16 & 1.192021 & -0.004312 & -0.684553 \\ 6 & 1.581041 & 1.392042 & 0.450371 \\ 1 & 0.883290 & 1.339176 & 1.286606 \\ 1 & 2.621356 & 1.306388 & 0.775476 \\ 1 & 1.438943 & 2.309670 & -0.123224 \\ 6 & -0.492720 & -0.007728 & -0.955928 \\ 1 & -0.770642 & -0.014813 & -2.001041 \\ 6 & -1.408126 & -0.000313 & 0.128252 \\ 8 & -1.072479 & 0.008485 & 1.332459 \\ 6 & -2.894707 & -0.001259 & -0.223551 \\ 1 & -3.086822 & -0.034736 & -1.300509 \\ 1 & -3.373970 & -0.863774 & 0.252669 \\ 1 & -3.361972 & 0.897846 & 0.193894\end{array}$

$\mathrm{E}(\mathrm{MeCN})=-670.0243436$

NImag $=0$

114. Enone (-270.5574584)

\begin{tabular}{|c|c|c|c|}
\hline 6 & -1.084072 & 0.148104 & -0.000008 \\
\hline 8 & -1.251023 & 1.359084 & 0.000008 \\
\hline 6 & 0.272311 & -0.467560 & -0.000010 \\
\hline 6 & 1.381227 & 0.285626 & -0.000019 \\
\hline 1 & 0.336491 & -1.555167 & 0.000011 \\
\hline 1 & 1.242659 & 1.366923 & -0.000008 \\
\hline 6 & 2.785878 & -0.227021 & 0.000010 \\
\hline 1 & 3.333288 & 0.140225 & 0.878704 \\
\hline 1 & 2.826325 & -1.320900 & 0.000031 \\
\hline 1 & 3.333307 & 0.140193 & -0.878687 \\
\hline 6 & -2.259623 & -0.814652 & -0.000002 \\
\hline 1 & -2.220652 & -1.467510 & 0.881651 \\
\hline 1 & -3.197086 & -0.255605 & -0.000171 \\
\hline 1 & -2.220479 & -1.467811 & -0.881419 \\
\hline
\end{tabular}

NImag $=0$ 


\begin{tabular}{|c|c|c|c|c|c|c|c|}
\hline \multicolumn{4}{|c|}{$\mathrm{HF} / 6-31 \mathrm{G}^{*}$ geometries computed for anti profile of } & \multicolumn{4}{|c|}{ 116. I-3c $(-1011.1550547)$} \\
\hline \multicolumn{4}{|c|}{ ylide $3\left(\mathrm{HF} / 6-31 \mathrm{G}^{*}\right.$ energies in parentheses, single - } & 16 & -0.438320 & 1.352274 & 0.445551 \\
\hline \multicolumn{4}{|c|}{ point energies at the $\mathrm{PCM}\left(\mathrm{CH}_{3} \mathrm{CN}\right) / \mathrm{B} 3 \mathrm{LYP} / 6-$} & 6 & 0.743276 & 2.043449 & 1.633017 \\
\hline \multirow{2}{*}{\multicolumn{4}{|c|}{$311+\mathrm{G}^{* *} / / \mathrm{HF} / 6-31 \mathrm{G}^{*}$ level is provided after the }} & 6 & -0.300698 & 2.549652 & -0.893053 \\
\hline & & & & 6 & 0.308907 & -0.182631 & -0.240575 \\
\hline \multicolumn{4}{|c|}{ optimized coordinates) } & 6 & -0.291598 & -1.426014 & 0.458277 \\
\hline \multirow{3}{*}{\multicolumn{4}{|c|}{ 115. TS-3c (-1011.1323889) }} & 6 & 0.171175 & -1.576815 & 1.915011 \\
\hline & & & & 6 & -1.800401 & -1.458210 & 0.340255 \\
\hline & & & & 6 & -2.563712 & -0.660664 & -0.453295 \\
\hline 6 & 0.8 & 2.782810 & -0.823 & 8 & -2.147978 & 0.318755 & -1.163422 \\
\hline 1 & 1.872426 & 2.657263 & $\begin{array}{l}-0.025 \\
-0.466\end{array}$ & 6 & -4.061718 & -0.898749 & -0.498849 \\
\hline 1 & 0.816355 & 2.638284 & -1.894548 & 1 & 0.926339 & 1.305234 & 2.400386 \\
\hline 16 & -0.233281 & 1.558842 & -0.075212 & 1 & 0.246886 & 2.893443 & 2.084045 \\
\hline 6 & 0.039415 & 1.865453 & 1.678737 & 1 & 1.669211 & 2.339098 & 1.171826 \\
\hline 1 & 1.075027 & 1.703102 & 1.927519 & 1 & -0.617756 & 3.505930 & -0.498677 \\
\hline 1 & -0.608003 & 1.191969 & 2.216751 & 1 & 0.718199 & 2.603337 & -1.244063 \\
\hline 6 & 0.452646 & -0.035639 & -0.427370 & 1 & -0.987009 & 2.210801 & -1.651584 \\
\hline 1 & 0.118808 & -0.293525 & -1.416683 & 1 & -0.101867 & -0.168012 & -1.240051 \\
\hline 6 & -0.691271 & -1.393188 & 0.486631 & 1 & 1.255011 & -1.574706 & 2.014540 \\
\hline 6 & 0.082044 & -1.863021 & 1.698442 & 1 & -0.244847 & -0.799069 & 2.549105 \\
\hline 1 & 0.110734 & -1.117732 & 2.485006 & 1 & -0.187451 & -2.521520 & 2.308141 \\
\hline 1 & -0.423488 & -2.738021 & 2.096476 & 1 & 0.145396 & -2.256401 & -0.092123 \\
\hline 1 & 1.097556 & -2.150205 & 1.457349 & 1 & -2.270601 & -2.253759 & 0.891686 \\
\hline 1 & -0.576167 & -2.036180 & -0.365809 & 1 & -4.577062 & 0.004274 & -0.183120 \\
\hline 6 & -1.996970 & -0.879594 & 0.672143 & 1 & -4.359052 & -1.089657 & -1.526065 \\
\hline 1 & -2.350530 & -0.652035 & 1.663030 & 1 & -4.387285 & -1.726595 & 0.121393 \\
\hline 6 & -2.810092 & -0.665738 & -0.464247 & 6 & 1.818004 & -0.126135 & -0.390200 \\
\hline 6 & -4.257909 & -0.249842 & -0.256205 & 8 & 2.469878 & 0.872957 & -0.408062 \\
\hline 1 & -4.522921 & -0.125173 & 0.787910 & 8 & 2.325071 & -1.318449 & -0.600211 \\
\hline 1 & -4.445038 & 0.678539 & -0.786686 & 6 & 3.717354 & -1.397420 & -0.875460 \\
\hline 1 & -4.903928 & -1.004304 & -0.693791 & 1 & 3.923259 & -2.444894 & -1.023286 \\
\hline 8 & -2.402950 & -0.782461 & -1.616839 & 1 & 3.955645 & -0.835228 & -1.765957 \\
\hline 6 & 1.889441 & -0.181994 & -0.204585 & 1 & 4.286808 & -1.013551 & -0.042213 \\
\hline 8 & 2.585098 & 0.515275 & 0.487603 & & & & \\
\hline 8 & 2.356298 & -1.255765 & -0.826653 & & & & \\
\hline & & & & & & & \\
\hline
\end{tabular}

$\begin{array}{lllll}1 & 4.353533 & -0.773633 & -0.995755\end{array}$

$\begin{array}{lllll}1 & 3.932068 & -1.749606 & 0.409033\end{array}$

$\begin{array}{lllll}1 & 3.896021 & -2.471700 & -1.208532\end{array}$

$\begin{array}{llll}1 & -0.258255 & 2.886451 & 1.876518\end{array}$

$\begin{array}{lllll}1 & 0.483468 & 3.766837 & -0.580799\end{array}$

$\mathrm{E}(\mathrm{MeCN})=-1015.8799457$

NImag $=1(-524.1)$

117.TS-3t (-1015.6640743)

$\begin{array}{cccc}6 & -2.495330 & -0.715804 & 1.522123 \\ 16 & -2.426474 & -0.493650 & -0.268182 \\ 6 & -3.405460 & 1.015195 & -0.475083 \\ 6 & -0.703911 & -0.184085 & -0.653194 \\ 6 & 0.473401 & -1.596136 & -0.025818 \\ 6 & -0.179327 & -2.853204 & -0.570878 \\ 6 & 1.805964 & -1.351269 & -0.432745 \\ 6 & 2.626975 & -0.519371 & 0.349933\end{array}$




\begin{tabular}{|c|c|c|c|c|c|c|c|}
\hline 8 & 2.240210 & 0.094850 & 1.344840 & 1 & 4.273045 & -1.293652 & -0.803695 \\
\hline 6 & 4.087176 & -0.369275 & -0.050148 & 1 & 4.551226 & -0.833274 & 0.879649 \\
\hline 1 & -1.983890 & -1.635712 & 1.760309 & 1 & 4.295567 & 0.406390 & -0.323671 \\
\hline 1 & -3.537510 & -0.800781 & 1.798136 & 6 & -0.078926 & 1.084082 & -0.033967 \\
\hline 1 & -2.020733 & 0.122340 & 2.005106 & 8 & -0.575467 & 1.594318 & 0.924663 \\
\hline 1 & -4.426331 & 0.786565 & -0.198255 & 8 & 0.770965 & 1.658813 & -0.835120 \\
\hline 1 & -2.999591 & 1.806568 & 0.133259 & 6 & 1.467702 & 2.792411 & -0.321623 \\
\hline 1 & -3.368135 & 1.279916 & -1.522613 & 1 & 2.159426 & 3.077118 & -1.098488 \\
\hline 1 & -0.605290 & -0.280720 & -1.721432 & 1 & 0.774356 & 3.597146 & -0.121989 \\
\hline 1 & 0.317753 & -1.429440 & 1.025627 & 1 & 1.988357 & 2.493054 & 0.572286 \\
\hline 1 & -1.217172 & -2.978630 & -0.277661 & & & & \\
\hline 1 & 0.371922 & -3.708571 & -0.195251 & \multicolumn{4}{|c|}{$E(\mathrm{MeCN})=-1015.8853116$} \\
\hline 1 & -0.130367 & -2.881288 & -1.654122 & \multicolumn{4}{|c|}{ NImag $=0$} \\
\hline 1 & 2.164552 & -1.768485 & -1.357172 & \multirow{2}{*}{\multicolumn{4}{|c|}{ 119. TS-3R (-1011.1244412) }} \\
\hline 1 & 4.359857 & -0.965557 & -0.913558 & & & & \\
\hline 1 & 4.712371 & -0.654114 & 0.790021 & & & & \\
\hline 1 & 4.294952 & 0.675362 & -0.263422 & 6 & 1.908658 & -0.521296 & 2.208207 \\
\hline 6 & -0.246798 & 1.134633 & -0.139949 & 1 & 2.185612 & 0.499956 & 1.999048 \\
\hline 8 & -0.733463 & 1.694652 & 0.804832 & 1 & 1.076127 & -0.545683 & 2.896720 \\
\hline 8 & 0.726943 & 1.630929 & -0.860431 & 16 & 1.371014 & -1.355703 & 0.700590 \\
\hline 6 & 1.413285 & 2.755810 & -0.324291 & 6 & 2.831320 & -1.208077 & -0.353589 \\
\hline 1 & 2.181553 & 2.991766 & -1.043990 & 1 & 3.214790 & -0.199017 & -0.337822 \\
\hline 1 & 0.736215 & 3.590435 & -0.212037 & 1 & 2.542287 & -1.471717 & -1.359012 \\
\hline \multirow[t]{2}{*}{1} & 1.848051 & 2.486325 & 0.624333 & 6 & 0.070136 & -0.223109 & 0.009273 \\
\hline & & & & 1 & -0.422196 & 0.121872 & 0.899317 \\
\hline \multicolumn{4}{|c|}{$\mathrm{E}(\mathrm{MeCN})=-1015.8760525$} & 6 & -0.977960 & -0.958253 & -0.907322 \\
\hline \multirow{2}{*}{\multicolumn{4}{|c|}{ NImag = $1(-322.0)$}} & 6 & -0.564507 & -2.340374 & -1.416611 \\
\hline & & & & 1 & -0.505846 & -3.076602 & -0.620446 \\
\hline \multirow{2}{*}{\multicolumn{4}{|c|}{ 118. I-3t (-1011.1368495) }} & 1 & -1.322798 & -2.683048 & -2.110678 \\
\hline & & & & 1 & 0.379392 & -2.331736 & -1.959444 \\
\hline 6 & -2.589364 & -0.594250 & 1.505025 & 1 & -1.032217 & -0.310051 & -1.775071 \\
\hline 16 & -2.372915 & -0.443949 & -0.283640 & 6 & -2.292231 & -0.979872 & -0.178739 \\
\hline 6 & -3.129116 & 1.157169 & -0.639396 & 1 & -2.866211 & -1.887828 & -0.139318 \\
\hline 6 & -0.532348 & -0.269373 & -0.581700 & 6 & -2.762625 & 0.232916 & 0.245906 \\
\hline 6 & 0.240583 & -1.519107 & -0.017953 & 6 & -4.130933 & 0.333040 & 0.896468 \\
\hline 6 & -0.384826 & -2.828650 & -0.512648 & 1 & -4.638200 & -0.623568 & 0.971225 \\
\hline 6 & 1.681282 & -1.433495 & -0.414833 & 1 & -4.031146 & 0.760106 & 1.890902 \\
\hline 6 & 2.508989 & -0.603997 & 0.294672 & 1 & -4.749942 & 1.014781 & 0.320239 \\
\hline 8 & 2.109640 & 0.170969 & 1.197961 & 8 & -2.090042 & 1.297650 & 0.153607 \\
\hline 6 & 3.995550 & -0.593888 & -0.022246 & 6 & 0.739092 & 0.978106 & -0.636493 \\
\hline 1 & -2.187907 & -1.551075 & 1.801363 & 8 & 1.019467 & 1.045485 & -1.787827 \\
\hline 1 & -3.653195 & -0.572082 & 1.698296 & 1 & 3.571918 & -1.904004 & 0.014696 \\
\hline 1 & -2.071020 & 0.210319 & 1.998728 & 1 & 2.738427 & -1.077832 & 2.621836 \\
\hline 1 & -4.198645 & 1.041525 & -0.529303 & 8 & 1.040722 & 1.908674 & 0.250210 \\
\hline 1 & -2.743569 & 1.911159 & 0.026654 & 6 & 1.439550 & 3.174469 & -0.258081 \\
\hline 1 & -2.901175 & 1.402887 & -1.667643 & 1 & 0.661516 & 3.571561 & -0.891352 \\
\hline 1 & -0.466508 & -0.272008 & -1.660360 & 1 & 1.577918 & 3.805424 & 0.605161 \\
\hline 1 & 0.167186 & -1.454446 & 1.062992 & 1 & 2.360164 & 3.088872 & -0.817476 \\
\hline 1 & -1.406239 & -2.999360 & -0.177880 & \multirow{4}{*}{\multicolumn{4}{|c|}{$\begin{array}{l}\mathrm{E}(\mathrm{MeCN})=-1015.8788289 \\
\text { NImag }=1(-58.7)\end{array}$}} \\
\hline 1 & 0.217387 & -3.650052 & -0.145272 & & & & \\
\hline 1 & -0.382142 & -2.878672 & -1.598220 & & & & \\
\hline 1 & 2.032225 & -2.070004 & -1.207211 & & & & \\
\hline
\end{tabular}


120. TS-3E (-1011.1323204)

$\begin{array}{crrr}16 & -2.474854 & -0.423601 & -0.382319 \\ 6 & -2.808339 & -0.596393 & 1.386373 \\ 6 & -3.118509 & 1.232857 & -0.711613 \\ 6 & -0.352161 & -0.346900 & -0.563420 \\ 6 & 0.279339 & -1.561060 & 0.086434 \\ 6 & -0.341718 & -2.889530 & -0.339949 \\ 6 & 1.683320 & -1.398343 & -0.418304 \\ 6 & 2.578667 & -0.577181 & 0.247987 \\ 8 & 2.278586 & 0.151594 & 1.209510 \\ 6 & 4.020731 & -0.543417 & -0.233469 \\ 1 & -2.446404 & -1.566094 & 1.694283 \\ 1 & -3.878604 & -0.550449 & 1.536003 \\ 1 & -2.297089 & 0.181964 & 1.928514 \\ 1 & -4.195681 & 1.211021 & -0.615887 \\ 1 & -2.688649 & 1.944030 & -0.023319 \\ 1 & -2.859037 & 1.491917 & -1.729219 \\ 1 & -0.323831 & -0.368706 & -1.637382 \\ 1 & -1.362846 & -3.027120 & 0.007216 \\ 1 & -0.343619 & -2.993001 & -1.421295 \\ 1 & 0.257386 & -3.695027 & 0.066922 \\ 1 & 0.247212 & -1.440311 & 1.162459 \\ 1 & 1.970293 & -1.968103 & -1.283709 \\ 1 & 4.281630 & 0.473391 & -0.513123 \\ 1 & 4.671032 & -0.824781 & 0.589018 \\ 1 & 4.210994 & -1.200827 & -1.074728 \\ 6 & -0.016078 & 1.018753 & 0.033099 \\ 8 & -0.507880 & 1.422287 & 1.038351 \\ 8 & 0.759648 & 1.710764 & -0.754915 \\ 6 & 1.346293 & 2.885481 & -0.198462 \\ 1 & 1.995148 & 3.272312 & -0.967937 \\ 1 & 0.580059 & 3.607337 & 0.045719 \\ 1 & 1.907577 & 2.607307 & 0.677943\end{array}$

$\mathrm{E}(\mathrm{MeCN})=-1015.8762933$

NImag $=1(-416.9)$

121. PC-3 (-1011.2518339)

$\begin{array}{cccc}16 & 3.510327 & -0.525224 & -0.378730 \\ 6 & 2.703893 & 0.310407 & -1.768247 \\ 6 & 3.569520 & 0.835444 & 0.814408 \\ 6 & -1.413346 & -0.103113 & 1.282450 \\ 6 & -0.440500 & -1.209604 & 1.190925 \\ 6 & -0.191608 & -2.136815 & 2.357439 \\ 6 & -1.737850 & -1.294591 & 0.410630 \\ 6 & -1.695379 & -1.171072 & -1.077539 \\ 8 & -1.011856 & -0.354048 & -1.620569 \\ 6 & -2.550566 & -2.140499 & -1.858454 \\ 1 & 2.594384 & -0.414907 & -2.564002 \\ 1 & 3.311757 & 1.132041 & -2.126898\end{array}$

$\begin{array}{rrr}1.724643 & 0.671815 & -1.487130 \\ 4.185448 & 1.646023 & 0.444438 \\ 2.574801 & 1.201953 & 1.028026 \\ 4.013360 & 0.451074 & 1.724133 \\ -2.015913 & -0.026284 & 2.172554 \\ 0.548185 & -1.710405 & 3.026927 \\ -1.099022 & -2.310966 & 2.927835 \\ 0.180808 & -3.096699 & 2.015516 \\ 0.421337 & -0.998679 & 0.582683 \\ -2.489986 & -1.961447 & 0.795553 \\ -3.597972 & -1.992869 & -1.610694 \\ -2.403488 & -1.987732 & -2.918226 \\ -2.295888 & -3.163064 & -1.595427 \\ -1.110136 & 1.243677 & 0.692142 \\ -0.033735 & 1.745206 & 0.652306 \\ -2.215058 & 1.845415 & 0.287851 \\ -2.066992 & 3.124724 & -0.303241 \\ -3.062755 & 3.442387 & -0.570439 \\ -1.627389 & 3.818888 & 0.398376 \\ -1.444566 & 3.058521 & -1.183315\end{array}$

$\mathrm{E}(\mathrm{MeCN})=-1015.9525788$

NImag $=0$

The optimized geometries in continuum solvent for diastereomeric TSs for ylide 5 [Energies at the $\underline{\mathrm{PCM}\left(\mathrm{CH}_{3}\right.} \underline{\mathrm{CN}) / \mathrm{B} 3 \mathrm{LYP} / 6-31 \mathrm{G}^{*} \text { level are given in }}$ parentheses; Single-point energies at the

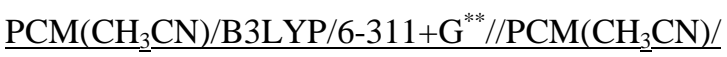
B3LYP/6-31G ${ }^{*}$ level are also provided after the respective geometries]

122. TS-5 $c(-940.4537031)$

$\begin{array}{rcc}1.834607 & 2.319126 & -0.902438 \\ 2.772256 & 1.847674 & -0.606699 \\ 1.677867 & 2.246443 & -1.981255 \\ 0.439529 & 1.452426 & -0.103648 \\ 0.902584 & 1.621053 & 1.651846 \\ 1.854169 & 1.117990 & 1.815717 \\ 0.099003 & 1.176894 & 2.239136 \\ 0.662301 & -0.275217 & -0.523712 \\ 0.139662 & -0.421205 & -1.469429 \\ -0.735806 & -1.288950 & 0.535794 \\ -0.045364 & -1.864141 & 1.755397 \\ 0.094273 & -1.120776 & 2.547184 \\ -0.677140 & -2.661302 & 2.169026 \\ 0.929735 & -2.303279 & 1.525191\end{array}$




\begin{tabular}{|c|c|c|c|c|c|c|c|}
\hline 1 & -0.831427 & -2.002287 & -0.283450 & \multirow[t]{2}{*}{1} & \multirow[t]{2}{*}{2.650882} & -2.793208 & 0.582487 \\
\hline 6 & -1.877858 & -0.467628 & 0.715134 & & & & \\
\hline 1 & -2.061338 & -0.004858 & 1.683903 & \multicolumn{4}{|c|}{$E(S P)=-940.6343322$} \\
\hline 6 & -2.740616 & -0.182449 & -0.373762 & \multicolumn{4}{|c|}{ NImag = $1(-329)$} \\
\hline 6 & -3.982111 & 0.668180 & -0.109289 & \\
\hline 1 & -4.097351 & 0.946124 & 0.943144 & \multirow{2}{*}{\multicolumn{4}{|c|}{ 124. I-5c (-940.4655427) }} \\
\hline 1 & -3.937721 & 1.584126 & -0.712036 & & & & \\
\hline 1 & -4.877131 & 0.121051 & -0.430419 & 6 & 1.164023 & 2.426625 & -0.872492 \\
\hline 8 & -2.536351 & -0.586074 & -1.550128 & 1 & 2.189607 & 2.237228 & -0.555567 \\
\hline 6 & 1.992355 & -0.848585 & -0.401580 & 1 & 1.036477 & 2.219635 & -1.938592 \\
\hline 8 & 2.897340 & -0.379004 & 0.310088 & 16 & -0.027422 & 21.358803 & 30.030414 \\
\hline 1 & 0.964128 & 2.692458 & 1.860019 & 6 & 0.520775 & 1.689664 & 1.740784 \\
\hline 1 & 1.793944 & 3.366709 & -0.591716 & 1 & 1.564571 & 1.405258 & 1.863613 \\
\hline 6 & 2.218995 & -2.138747 & -1.176856 & 1 & -0.138968 & 1.125394 & 2.399859 \\
\hline 1 & 1.312402 & -2.749377 & -1.251217 & 6 & 0.569412 & -0.333242 & -0.446951 \\
\hline 1 & 2.533167 & -1.894939 & -2.200632 & 1 & 0.185423 & -0.373522 & -1.472888 \\
\hline \multirow[t]{2}{*}{1} & 3.016598 & -2.715492 & -0.700984 & 6 & -0.283507 & -1.404648 & 0.353588 \\
\hline & & & & 6 & 0.405841 & -1.916280 & 1.627207 \\
\hline \multicolumn{4}{|c|}{$E(S P)=-940.6328023$} & 1 & 0.558781 & -1.123281 & 2.366963 \\
\hline \multirow{2}{*}{\multicolumn{4}{|c|}{ NImag = $1(-363.3)$}} & 1 & -0.231546 & -2.676708 & 2.091400 \\
\hline & & & & 1 & 1.378678 & -2.379080 & 1.426352 \\
\hline \multirow{2}{*}{\multicolumn{4}{|c|}{ 123. TS-5 $c$ conformer2 $(-940.4550373)$}} & 1 & -0.338736 & -2.246144 & -0.359614 \\
\hline & & & & 6 & -1.652369 & -0.850256 & 0.633318 \\
\hline 6 & -0.194903 & 2.231379 & -1.356909 & 1 & -2.029361 & -0.923351 & 1.650861 \\
\hline 16 & 0.483636 & 1.436042 & 0.130609 & 6 & -2.472905 & -0.407998 & -0.398269 \\
\hline 6 & 1.114404 & -0.121374 & -0.508778 & 6 & -3.879539 & 0.081604 & -0.054127 \\
\hline 6 & 1.900923 & -0.776834 & 0.522442 & 1 & -4.126660 & -0.026060 & 1.007499 \\
\hline 6 & 2.905417 & -1.839071 & 0.106988 & 1 & -3.982742 & 1.138931 & -0.332311 \\
\hline 6 & 1.975184 & 2.453769 & 0.414018 & 1 & -4.621070 & -0.472117 & -0.644806 \\
\hline 8 & 1.747310 & -0.505580 & 1.725796 & 8 & -2.108181 & -0.332660 & -1.623954 \\
\hline 6 & -0.584544 & -1.213874 & -0.865103 & 6 & 2.064199 & -0.564816 & -0.390148 \\
\hline 6 & -1.510688 & -1.162442 & 0.202104 & 8 & 2.830264 & 0.110176 & 0.286022 \\
\hline 6 & -2.563177 & -0.214618 & 0.249937 & 1 & 0.379068 & 2.761889 & 1.905218 \\
\hline 8 & -2.767743 & 0.691719 & -0.607286 & 1 & 0.871920 & 3.460187 & -0.668780 \\
\hline 6 & 0.001439 & -2.553898 & -1.262540 & 6 & 2.554752 & -1.730160 & -1.223166 \\
\hline 6 & -3.550444 & -0.311851 & 1.412927 & 1 & 1.896406 & -2.601336 & -1.131966 \\
\hline 1 & 0.490567 & 2.119223 & -2.200802 & 1 & 2.553277 & -1.438294 & -2.282260 \\
\hline 1 & -1.162911 & 1.748644 & -1.523086 & 1 & 3.572876 & -1.992569 & -0.926620 \\
\hline 1 & 2.578201 & 2.506440 & -0.495455 & & & & \\
\hline 1 & 2.527526 & 1.978001 & 1.225463 & \multicolumn{4}{|c|}{$E(S P)=-940.6457203$} \\
\hline 1 & 1.560003 & -0.013729 & -1.500298 & \multicolumn{4}{|c|}{ NImag $=0$} \\
\hline 1 & 0.338648 & -3.126825 & -0.392418 & & & & \\
\hline 1 & -0.784350 & -3.143440 & -1.753145 & \multirow{2}{*}{\multicolumn{4}{|c|}{ 125. TS-5 $t(-940.4501561)$}} \\
\hline 1 & 0.834017 & -2.461963 & -1.967344 & & & & \\
\hline 1 & -0.869826 & -0.605706 & -1.724389 & 6 & 3.511793 & -0.791054 & -0.423096 \\
\hline 1 & -1.422055 & -1.886397 & 1.010103 & 1 & 3.157705 & -1.663180 & 0.125737 \\
\hline 1 & -3.339820 & -1.146252 & 2.088882 & 1 & 3.585834 & -0.995709 & -1.493554 \\
\hline 1 & -3.530389 & 0.621665 & 1.989515 & 16 & 2.312868 & 0.583308 & -0.229076 \\
\hline 1 & -4.569663 & -0.419749 & 1.021674 & 6 & 2.299341 & 0.744864 & 1.588826 \\
\hline 1 & 1.643074 & 3.450013 & 0.719339 & 1 & 1.972120 & -0.198564 & 2.023616 \\
\hline 1 & -0.338976 & 3.287980 & -1.115663 & 1 & 1.618446 & 1.557793 & 1.846168 \\
\hline 1 & 2.965279 & -1.982675 & -0.974099 & 6 & 0.677344 & -0.014251 & -0.675647 \\
\hline 1 & 3.893404 & -1.544933 & 0.480901 & 1 & 0.580797 & 0.151205 & -1.750768 \\
\hline
\end{tabular}




\begin{tabular}{|c|c|c|c|c|c|c|c|}
\hline 6 & -0.720161 & 1.279560 & -0.057447 & 6 & 0.672064 & 1.156038 & -0.997054 \\
\hline 6 & -0.202674 & 2.596610 & -0.614634 & 8 & 0.992178 & 0.448240 & -1.938245 \\
\hline 1 & -0.271763 & 2.619805 & -1.708303 & 1 & 1.370340 & -3.073185 & -0.026337 \\
\hline 1 & -0.826789 & 3.412150 & -0.228915 & 1 & 3.909046 & -1.531641 & -0.074630 \\
\hline 1 & 0.832728 & 2.823059 & -0.337983 & 6 & 0.263996 & 2.599446 & -1.205240 \\
\hline 1 & -0.545422 & 1.129277 & 1.008092 & 1 & -0.323922 & 3.009133 & -0.382799 \\
\hline 6 & -2.024952 & 0.889910 & -0.456241 & 1 & 1.185214 & 3.193734 & -1.293353 \\
\hline 1 & -2.417969 & 1.245440 & -1.407490 & 1 & -0.288611 & 2.688529 & -2.143747 \\
\hline 6 & -2.831835 & 0.068910 & 0.371007 & & & & \\
\hline 6 & -4.269509 & -0.204185 & -0.077910 & \multicolumn{4}{|c|}{$E(S P)=-940.6421927$} \\
\hline 1 & -4.530110 & 0.286655 & -1.021178 & \multicolumn{4}{|c|}{ NImag $=0$} \\
\hline 1 & -4.420999 & -1.285197 & -0.189797 & & & & \\
\hline 1 & -4.966746 & 0.133298 & 0.699196 & & & & \\
\hline 8 & -2.444698 & -0.442309 & 1.454661 & \multirow{2}{*}{\multicolumn{4}{|c|}{ 127. TS-5R (-940.4508265) }} \\
\hline 6 & 0.356547 & -1.381792 & -0.220609 & & & & \\
\hline 8 & 0.875466 & -1.904964 & 0.773201 & 6 & 2.854963 & 0.289174 & 1.736326 \\
\hline 1 & 3.316107 & 1.007228 & 1.891952 & 1 & 3.097186 & 1.208984 & 1.201785 \\
\hline 1 & 4.474224 & -0.436204 & -0.043798 & 1 & 2.211691 & 0.487411 & 2.597268 \\
\hline 6 & -0.671145 & -2.106653 & -1.057924 & 16 & 1.956295 & -0.857354 & 0.638709 \\
\hline 1 & -1.526794 & -1.447044 & -1.249509 & 6 & 3.097589 & -0.911685 & -0.791964 \\
\hline 1 & -0.237523 & -2.369485 & -2.031723 & 1 & 3.159001 & 0.075746 & -1.244037 \\
\hline 1 & -0.994380 & -3.016781 & -0.547368 & 1 & 2.722613 & -1.657640 & -1.491743 \\
\hline \multirow{2}{*}{\multicolumn{4}{|c|}{$E(S P)=-940.6269246$}} & 6 & 0.416562 & 0.126465 & 0.225851 \\
\hline & & & & 1 & 0.003157 & 0.230683 & 1.236383 \\
\hline \multirow{2}{*}{\multicolumn{4}{|c|}{ NImag = 1(-355.6) }} & 6 & -0.695680 & -0.655500 & -0.639982 \\
\hline & & & & 6 & -0.133668 & -1.858277 & -1.416962 \\
\hline \multirow{2}{*}{\multicolumn{4}{|c|}{ 126. I-5t (-940.4624146) }} & 1 & 0.241016 & -2.647632 & -0.751806 \\
\hline & & & & 1 & -0.948035 & -2.296938 & -2.001254 \\
\hline & & & & 1 & 0.659789 & -1.581220 & -2.121909 \\
\hline 6 & 3.331405 & -0.611412 & -0.196747 & 1 & -1.025272 & 0.083521 & -1.381496 \\
\hline 1 & 3.134552 & -0.400214 & -1.247083 & 6 & -1.898933 & -0.979545 & 0.185063 \\
\hline 1 & 3.855541 & 0.217596 & 0.285771 & 1 & -1.868743 & -1.872425 & 0.808758 \\
\hline 16 & 1.760184 & -0.855746 & 0.693684 & 6 & -3.025966 & -0.177420 & 0.155782 \\
\hline 6 & 0.901132 & -2.122530 & -0.294703 & 6 & -4.228566 & -0.610223 & 1.001522 \\
\hline 1 & 1.021306 & -1.893905 & -1.352179 & 1 & -4.063904 & -1.546026 & 1.547607 \\
\hline 1 & -0.168573 & -2.105323 & 0.001917 & 1 & -4.478158 & 0.177744 & 1.725337 \\
\hline 6 & 0.810914 & 0.715374 & 0.468458 & 1 & -5.111325 & -0.731463 & 0.358864 \\
\hline 1 & 1.453533 & 1.440058 & 0.997289 & 8 & -3.170769 & 0.905664 & -0.525524 \\
\hline 6 & -0.598639 & 0.568016 & 1.182171 & 6 & 0.692893 & 1.515294 & -0.358852 \\
\hline 6 & -0.762403 & 1.670461 & 2.244368 & 8 & 1.707974 & 1.777444 & -0.987417 \\
\hline 1 & -0.814639 & 2.668210 & 1.790480 & 1 & 4.058527 & -1.240249 & -0.385876 \\
\hline 1 & -1.698247 & 1.511476 & 2.789999 & 1 & 3.761660 & -0.224964 & 2.065426 \\
\hline 1 & 0.062657 & 1.674124 & 2.968304 & 6 & -0.410026 & 2.513071 & -0.122803 \\
\hline 1 & -0.590756 & -0.397835 & 1.707533 & 1 & -1.404541 & 2.071032 & -0.313088 \\
\hline 6 & -1.735026 & 0.496745 & 0.196770 & 1 & -0.387634 & 2.812533 & 0.935004 \\
\hline 1 & -2.146119 & 1.428244 & -0.184451 & 1 & -0.240385 & 3.395589 & -0.744928 \\
\hline 6 & -2.364612 & -0.699805 & -0.092616 & \multirow{5}{*}{\multicolumn{4}{|c|}{$\begin{array}{l}E(S P)=-940.6300138 \\
\text { NImag }=1(-44.9)\end{array}$}} \\
\hline 6 & -3.558977 & -0.670737 & -1.049314 & & & & \\
\hline 1 & -3.830222 & 0.340001 & $\begin{array}{l}-1.373753 \\
-1039588\end{array}$ & & & & \\
\hline 1 & $\begin{array}{l}-3.336606 \\
-4.432809\end{array}$ & $\begin{array}{l}-1.274447 \\
-1.132650\end{array}$ & $\begin{array}{l}-1.939588 \\
-0.570206\end{array}$ & & & & \\
\hline $\begin{array}{l}1 \\
8\end{array}$ & $\begin{array}{l}-4.432809 \\
-2.027647\end{array}$ & $\begin{array}{l}-1.132650 \\
-1.858185\end{array}$ & 0.364662 & & & & \\
\hline
\end{tabular}


128. TS-5E (-940.3371092) [mPW1PW91/6-31G geometry]

$\begin{array}{cccc}6 & 3.149067 & -1.221198 & -0.210992 \\ 16 & 2.410388 & 0.425563 & -0.276112 \\ 6 & 0.434275 & 0.099125 & -0.686218 \\ 6 & -0.417997 & 1.256430 & -0.248717 \\ 6 & -1.710136 & 0.597997 & -0.642627 \\ 6 & -2.657837 & 0.199826 & 0.317423 \\ 8 & -2.517589 & 0.331169 & 1.558581 \\ 6 & 2.338020 & 0.834345 & 1.480556 \\ 6 & -0.017036 & -1.192633 & -0.029016 \\ 8 & 0.031475 & -1.317991 & 1.190543 \\ 6 & -0.338535 & -2.365634 & -0.924119 \\ 6 & -0.107219 & 2.596524 & -0.899870 \\ 6 & -3.935235 & -0.453663 & -0.194361 \\ 1 & 2.594707 & -1.856446 & 0.481476 \\ 1 & 3.126610 & -1.636092 & -1.220518 \\ 1 & 1.668884 & 0.135623 & 1.983789 \\ 1 & 1.963016 & 1.854970 & 1.567174 \\ 1 & 0.502882 & 0.022194 & -1.777543 \\ 1 & -0.131381 & 2.516662 & -1.992108 \\ 1 & -0.856754 & 3.335061 & -0.601964 \\ 1 & 0.877310 & 2.988923 & -0.616615 \\ 1 & -0.421853 & 1.327532 & 0.843621 \\ 1 & -1.938459 & 0.520483 & -1.702852 \\ 1 & -3.982754 & -0.517267 & -1.284546 \\ 1 & -4.018995 & -1.464772 & 0.219547 \\ 1 & -4.804721 & 0.107837 & 0.164471 \\ 1 & 3.352224 & 0.787818 & 1.882401 \\ 1 & 4.186829 & -1.118095 & 0.113332 \\ 1 & -0.735973 & -2.057697 & -1.891724 \\ 1 & 0.573111 & -2.953295 & -1.097318 \\ 1 & -1.056172 & -3.014599 & -0.418183\end{array}$

$E(S P)=-940.637077$

NImag $=1(-220.5)$

129. TS-5E (syn) (-940.435818492)

$\begin{array}{cccc}6 & -0.060366 & 2.530884 & 0.629956 \\ 16 & -0.951604 & 1.500281 & -0.576130 \\ 6 & -2.676085 & 1.997445 & -0.228203 \\ 6 & -0.419781 & -0.706806 & 0.445122 \\ 6 & 0.699453 & -1.375824 & -0.303530 \\ 6 & 0.316515 & -2.198057 & -1.538030 \\ 6 & -1.838443 & -1.154539 & 0.327603 \\ 8 & -2.446715 & -1.202346 & -0.734602 \\ 6 & -2.459550 & -1.620240 & 1.633281 \\ 6 & 1.666264 & -0.253232 & -0.560807 \\ 6 & 2.812862 & -0.104083 & 0.239190 \\ 6 & 3.831349 & 0.967098 & -0.150544\end{array}$

$\begin{array}{rrr}3.063590 & -0.831523 & 1.243630 \\ -0.548416 & 2.489142 & 1.608198 \\ 0.949087 & 2.117459 & 0.694532 \\ -2.908884 & 1.877866 & 0.833808 \\ -3.321793 & 1.352944 & -0.827058 \\ -0.117489 & -0.343765 & 1.427138 \\ -0.150323 & -1.576557 & -2.308504 \\ 1.222577 & -2.643507 & -1.961328 \\ -0.380760 & -3.005920 & -1.290093 \\ 1.192019 & -2.028746 & 0.439597 \\ 1.458072 & 0.427397 & -1.381699 \\ 3.542233 & 1.534211 & -1.041170 \\ 3.972443 & 1.667423 & 0.682438 \\ 4.803936 & 0.494390 & -0.334864 \\ -2.817931 & 3.041450 & -0.522423 \\ -0.015333 & 3.563333 & 0.273627 \\ -1.859872 & -2.417857 & 2.089705 \\ -2.490498 & -0.793835 & 2.355298 \\ -3.471700 & -1.984842 & 1.444422\end{array}$

$\mathrm{E}(\mathrm{SP})=-940.6155992$

NImag $=-235.8$

130. PC-5 (-940.5321352)

$\begin{array}{cccc}6 & -4.013601 & -1.354644 & -0.421387 \\ 16 & -4.310078 & 0.298180 & 0.303135 \\ 6 & 1.065463 & 0.288140 & 0.871180 \\ 6 & 1.390002 & 1.409221 & -0.048602 \\ 6 & 2.531148 & 0.536800 & 0.487793 \\ 6 & 3.253128 & -0.370180 & -0.448682 \\ 8 & 2.663739 & -1.080250 & -1.255485 \\ 6 & -3.261621 & 1.311458 & -0.802035 \\ 6 & 1.277318 & 2.855209 & 0.379972 \\ 6 & 4.765508 & -0.347833 & -0.363283 \\ 1 & -2.945296 & -1.588141 & -0.410952 \\ 1 & -4.554372 & -2.080515 & 0.191919 \\ 1 & -2.230934 & 0.948951 & -0.774242 \\ 1 & -3.300671 & 2.340612 & -0.434392 \\ 1 & 0.863961 & 0.523639 & 1.919349 \\ 1 & 1.544072 & 2.978186 & 1.436129 \\ 1 & 1.937851 & 3.497560 & -0.213930 \\ 1 & 0.250073 & 3.215887 & 0.248884 \\ 1 & 1.143484 & 1.202234 & -1.090498 \\ 1 & 3.143089 & 0.985901 & 1.269599 \\ 1 & 5.138817 & 0.672325 & -0.518034 \\ 1 & 5.092434 & -0.656147 & 0.638225 \\ 1 & 5.195677 & -1.017248 & -1.111296 \\ 1 & -3.639426 & 1.285451 & -1.828756 \\ 1 & -4.392252 & -1.402071 & -1.446929 \\ 6 & 0.300555 & -0.922325 & 0.388590 \\ 8 & -0.425896 & -0.880331 & -0.592960 \\ 6 & 0.426803 & -2.168295 & 1.237848\end{array}$




\begin{tabular}{|c|c|c|c|c|c|c|c|}
\hline 1 & 1.471810 & -2.500422 & 1.262869 & 6 & -1.099772 & -0.218032 & 0.465966 \\
\hline 1 & 0.135552 & -1.952355 & 2.273578 & 1 & -1.463569 & -0.244820 & 1.493840 \\
\hline \multirow[t]{2}{*}{1} & -0.203600 & -2.965397 & 0.837506 & 6 & 0.574685 & -1.391714 & 0.500763 \\
\hline & & & & 6 & 0.236967 & -2.296667 & 1.669583 \\
\hline \multicolumn{4}{|c|}{$E(S P)=-940.7013342$} & 1 & 0.925708 & -3.151818 & 1.669924 \\
\hline \multirow{2}{*}{\multicolumn{4}{|c|}{ NImag $=1(-1.5)$}} & 1 & 0.362680 & -1.775564 & 2.625819 \\
\hline & & & & 1 & -0.782315 & -2.692198 & 1.626201 \\
\hline \multirow{2}{*}{\multicolumn{4}{|c|}{ 131. TS-5'c (-940.4551312) }} & 6 & 1.777596 & -0.644250 & 0.554750 \\
\hline & & & & 1 & 2.205696 & -0.402995 & 1.527350 \\
\hline 6 & -1.896635 & -2.352263 & -0.937254 & 6 & 2.491055 & -0.276056 & -0.620706 \\
\hline 1 & -2.669592 & -2.138839 & -0.197721 & 6 & 3.835275 & 0.433593 & -0.436872 \\
\hline 1 & -2.226406 & -2.083293 & -1.943359 & 1 & 4.106050 & 0.588798 & 0.612354 \\
\hline 16 & -0.407627 & -1.360253 & -0.567987 & 1 & 4.626339 & -0.151161 & -0.922611 \\
\hline 6 & -0.068591 & -1.881762 & 1.145943 & 1 & 3.807364 & 1.407499 & -0.941376 \\
\hline 1 & -0.922013 & -1.611836 & 1.765825 & 8 & 2.105036 & -0.508761 & -1.792932 \\
\hline 1 & 0.858772 & -1.374845 & 1.439330 & 6 & -1.964862 & -0.737066 & -0.578448 \\
\hline 6 & -0.982466 & 0.335185 & -0.502272 & 8 & -1.970258 & -0.261853 & -1.726713 \\
\hline 1 & -0.947901 & 0.710201 & -1.525954 & 1 & -1.598439 & 3.476230 & -0.380504 \\
\hline 6 & 0.645009 & 1.391466 & 0.214558 & 1 & 0.385531 & 3.083838 & 1.484415 \\
\hline 6 & 0.144243 & 2.804382 & -0.008481 & 6 & -2.787467 & -1.972667 & -0.255184 \\
\hline 1 & 0.873499 & 3.502314 & 0.423502 & 1 & -3.054766 & -2.040627 & 0.803390 \\
\hline 1 & 0.058947 & 3.043010 & -1.074880 & 1 & -3.694505 & -1.968175 & -0.866393 \\
\hline 1 & -0.819089 & 2.994318 & 0.471838 & 1 & -2.213098 & -2.871066 & -0.519228 \\
\hline 6 & 1.832229 & 0.993493 & -0.441999 & 1 & 0.389957 & -1.824347 & -0.482512 \\
\hline 1 & 2.058181 & 1.435440 & -1.411635 & \multirow{2}{*}{\multicolumn{4}{|c|}{$E(S P)=-940.6293515$}} \\
\hline 6 & 2.782244 & 0.107049 & 0.128397 & & & & \\
\hline 6 & 4.070184 & -0.143863 & -0.655739 & \multicolumn{4}{|c|}{ NImag = $1(-348.2)$} \\
\hline 1 & 4.126844 & 0.424073 & -1.589296 & \multirow{2}{*}{\multicolumn{4}{|c|}{ 133. TS-5't $t(-940.4521031)$}} \\
\hline 1 & 4.935231 & 0.111131 & -0.031392 & & & & \\
\hline 1 & 4.152439 & -1.213100 & -0.888655 & & & & \\
\hline 8 & 2.665008 & -0.468452 & 1.246192 & 6 & -2.934483 & -1.025678 & -1.375250 \\
\hline 6 & -2.217933 & 0.590169 & 0.232666 & 16 & -1.657449 & -1.242571 & -0.080725 \\
\hline 8 & -2.577385 & -0.065831 & 1.223894 & 6 & -0.415524 & 0.028951 & -0.347624 \\
\hline 1 & 0.085039 & -2.964060 & 1.107979 & 6 & -0.893547 & 1.378493 & -0.644565 \\
\hline 1 & -1.604393 & -3.405584 & -0.911863 & 6 & 0.115679 & 2.275411 & -1.332623 \\
\hline 6 & -3.090425 & 1.741526 & -0.252374 & 6 & -2.615302 & -0.773481 & 1.398337 \\
\hline 1 & -2.618545 & 2.354677 & -1.023465 & 8 & -2.013529 & 1.800487 & -0.317539 \\
\hline 1 & -4.012963 & 1.315213 & -0.667839 & 6 & 0.884212 & 0.078475 & 1.172390 \\
\hline 1 & 0.564107 & 1.054635 & 1.248714 & 6 & 2.184550 & 0.320989 & 0.651463 \\
\hline \multirow[t]{2}{*}{1} & -3.378258 & 2.372561 & 0.595299 & 6 & 2.843717 & -0.648845 & -0.136438 \\
\hline & & & & 8 & 2.313443 & -1.744131 & -0.476917 \\
\hline \multicolumn{4}{|c|}{$E(S P)=-940.6342485$} & 6 & 0.350213 & 1.068566 & 2.190693 \\
\hline \multirow{2}{*}{\multicolumn{4}{|c|}{ NImag $=1(-344.0)$}} & 6 & 4.262010 & -0.353640 & -0.622639 \\
\hline & & & & 1 & -3.288005 & 0.005272 & -1.360684 \\
\hline \multirow{2}{*}{\multicolumn{4}{|c|}{ 132. TS-5' $c$ conformer2(-940.4524592) }} & 1 & -2.462626 & -1.271405 & -2.329312 \\
\hline & & & & 1 & -2.939369 & 0.261781 & 1.302260 \\
\hline 6 & 0.082060 & 2.044287 & 1.632736 & 1 & -1.973989 & -0.919553 & 2.268657 \\
\hline 1 & -0.732789 & 1.979688 & 2.358695 & 1 & 0.303476 & -0.422335 & -1.029454 \\
\hline 1 & 0.937679 & 1.445607 & 1.942533 & 1 & 0.870521 & 0.916616 & 3.145237 \\
\hline 16 & -0.464791 & 1.404647 & 0.017992 & 1 & 0.539163 & 2.101124 & 1.876964 \\
\hline 6 & -1.945956 & 2.452022 & -0.218499 & 1 & -0.721372 & 0.968015 & 2.377837 \\
\hline 1 & -2.593245 & 2.394778 & 0.659431 & 1 & 2.635438 & 1.302068 & 0.790273 \\
\hline 1 & -2.453333 & 2.076187 & -1.106562 & 1 & 4.641865 & 0.614238 & -0.280065 \\
\hline
\end{tabular}




$\begin{array}{rrrr}1 & 4.941378 & -1.141573 & -0.273619 \\ 1 & 4.290494 & -0.375592 & -1.719475 \\ 1 & -3.462744 & -1.462176 & 1.451895 \\ 1 & -3.740501 & -1.733935 & -1.164346 \\ 1 & 1.118741 & 2.126409 & -0.912519 \\ 1 & 0.175688 & 2.015023 & -2.397487 \\ 1 & -0.192651 & 3.320117 & -1.239724 \\ 1 & 0.695197 & -0.969247 & 1.418342\end{array}$

$\mathrm{E}(\mathrm{SP})=-940.6306427$

NImag $=1(-364.5)$

134. TS-5' $t$ conformer2 (-940.4557678)

$\begin{array}{crrr}6 & -0.786300 & -2.560714 & 0.121060 \\ 1 & -0.933404 & -2.866066 & -0.918163 \\ 1 & 0.276526 & -2.347618 & 0.289564 \\ 16 & -1.716995 & -1.019756 & 0.429003 \\ 6 & -3.141634 & -1.241730 & -0.700431 \\ 1 & -2.782143 & -1.461277 & -1.708504 \\ 1 & -3.700461 & -0.305939 & -0.675708 \\ 6 & -0.610024 & 0.185098 & -0.306654 \\ 1 & -0.011280 & -0.250105 & -1.103189 \\ 6 & 0.875734 & 0.483516 & 1.075066 \\ 6 & 0.359685 & 1.583639 & 1.977818 \\ 1 & 0.941052 & 1.581629 & 2.909088 \\ 1 & 0.477477 & 2.572357 & 1.520636 \\ 1 & -0.694016 & 1.452133 & 2.242357 \\ 6 & 2.104549 & 0.651441 & 0.397226 \\ 1 & 2.507190 & 1.652567 & 0.254382 \\ 6 & 2.773158 & -0.460344 & -0.168883 \\ 6 & 4.120323 & -0.225482 & -0.848719 \\ 1 & 4.446493 & 0.818283 & -0.804237 \\ 1 & 4.885319 & -0.855407 & -0.377798 \\ 1 & 4.062509 & -0.531919 & -1.900918 \\ 8 & 2.323684 & -1.641804 & -0.149413 \\ 6 & -1.269012 & 1.455397 & -0.536529 \\ 8 & -2.356913 & 1.746139 & -0.008396 \\ 1 & -3.746997 & -2.064602 & -0.310264 \\ 1 & -1.164253 & -3.323897 & 0.804374 \\ 6 & -0.519436 & 2.442629 & -1.409497 \\ 1 & 0.562133 & 2.381414 & -1.239715 \\ 1 & -0.695513 & 2.206775 & -2.467249 \\ 1 & -0.879685 & 3.456316 & -1.214556 \\ 1 & 0.744553 & -0.513934 & 1.495569\end{array}$

$\mathrm{E}(\mathrm{SP})=-940.634487$

NImag $=1(-336.2)$

135. I-5't (-940.4670633)

$\begin{array}{cllll}6 & -1.227737 & -2.406131 & 0.597038 \\ 16 & -1.941380 & -0.734730 & 0.349013\end{array}$

$\begin{array}{lrrr}6 & -2.703859 & -0.947060 & -1.293794 \\ 6 & -0.380053 & 0.220125 & -0.056706 \\ 6 & 0.619520 & 0.218714 & 1.177938 \\ 6 & 0.279363 & 1.290079 & 2.221344 \\ 6 & -0.817327 & 1.586102 & -0.526588 \\ 8 & -1.983017 & 1.953227 & -0.398104 \\ 6 & 0.235233 & 2.451537 & -1.166869 \\ 6 & 2.019889 & 0.301671 & 0.649367 \\ 6 & 2.482297 & -0.649488 & -0.241260 \\ 6 & 3.900103 & -0.516300 & -0.794332 \\ 8 & 1.781548 & -1.647818 & -0.668422 \\ 1 & -0.248565 & -2.450254 & 0.106060 \\ 1 & -1.131021 & -2.565869 & 1.672305 \\ 1 & -1.962418 & -1.326889 & -2.001552 \\ 1 & -3.082144 & 0.027564 & -1.601653 \\ 1 & 0.120343 & -0.389147 & -0.823743 \\ 1 & 0.933826 & 1.169963 & 3.091405 \\ 1 & 0.438310 & 2.303052 & 1.832385 \\ 1 & -0.758692 & 1.220176 & 2.568312 \\ 1 & 2.640936 & 1.142077 & 0.949989 \\ 1 & 4.441312 & 0.342493 & -0.382383 \\ 1 & 4.474604 & -1.427644 & -0.580561 \\ 1 & 3.867386 & -0.417094 & -1.887702 \\ 1 & -3.529067 & -1.654720 & -1.180364 \\ 1 & -1.934349 & -3.129507 & 0.182958 \\ 1 & 1.199332 & 2.335044 & -0.659605 \\ 1 & 0.387436 & 2.110175 & -2.200175 \\ 1 & -0.098195 & 3.492397 & -1.183306 \\ 1 & 0.470222 & -0.764897 & 1.648795 \\ \mathrm{E}(\mathrm{SP})=-940.6457746 & & \\ \mathrm{NImag}=0 & & & \\ & & & \end{array}$

136. TS-5'R (-940.4559843)

$\begin{array}{cccc}6 & 0.835045 & -2.494669 & 0.787403 \\ 1 & 1.311351 & -2.179643 & 1.719415 \\ 1 & -0.257423 & -2.496336 & 0.854700 \\ 16 & 1.194704 & -1.300197 & -0.538610 \\ 6 & 3.011858 & -1.162627 & -0.394437 \\ 1 & 3.312511 & -0.901815 & 0.620526 \\ 1 & 3.351003 & -0.403158 & -1.097282 \\ 6 & 0.359547 & 0.239390 & 0.129476 \\ 1 & -0.269795 & -0.191621 & 0.904237 \\ 6 & -0.647002 & 0.813668 & -0.995116 \\ 6 & -0.213711 & 2.165987 & -1.578390 \\ 1 & -0.883942 & 2.421951 & -2.406089 \\ 1 & -0.282944 & 2.977980 & -0.844517 \\ 1 & 0.810191 & 2.145607 & -1.967048 \\ 6 & -2.031721 & 0.808718 & -0.431615 \\ 1 & -2.578384 & 1.743707 & -0.337405 \\ 6 & -2.562275 & -0.400576 & -0.030956 \\ 6 & -3.964988 & -0.449271 & 0.567579\end{array}$




\begin{tabular}{|c|c|c|c|c|c|c|c|}
\hline 1 & -4.450581 & 0.532485 & 0.595088 & \multicolumn{4}{|c|}{$E(S P)=-940.63812$} \\
\hline 1 & -4.598733 & -1.136333 & -0.009455 & \multicolumn{4}{|c|}{ NImag $=1(-266.5)$} \\
\hline 1 & -3.924419 & -0.847768 & 1.590467 & \multirow{3}{*}{\multicolumn{4}{|c|}{ 138. TS-5'E (syn) (-940.4389185) }} \\
\hline 8 & -1.917295 & -1.519538 & -0.120016 & & & & \\
\hline 6 & 1.268935 & 1.307352 & 0.702921 & & & & \\
\hline 8 & 2.388422 & 1.546854 & 0.275444 & 6 & -1.838706 & -1.989645 & -1.285770 \\
\hline 1 & 3.396188 & -2.146893 & -0.677809 & 1 & -2.731423 & -1.646382 & -0.757515 \\
\hline 1 & 1.216212 & -3.467161 & 0.465658 & 1 & -1.781850 & -1.510431 & -2.266747 \\
\hline 6 & 0.631345 & 2.106177 & 1.818202 & 16 & -0.303326 & -1.594819 & -0.373506 \\
\hline 1 & -0.432763 & 2.278551 & 1.616954 & 6 & -0.755383 & -2.260639 & 1.257316 \\
\hline 1 & 0.688074 & 1.524868 & 2.749101 & 1 & -1.441977 & -1.569300 & 1.756715 \\
\hline 1 & 1.162366 & 3.051264 & 1.955559 & 1 & 0.175974 & -2.322397 & 1.819315 \\
\hline \multirow[t]{2}{*}{1} & -0.575377 & 0.074053 & -1.808171 & 6 & -0.526739 & 0.971391 & -0.465973 \\
\hline & & & & 1 & -0.596024 & 0.980428 & -1.554114 \\
\hline \multicolumn{4}{|c|}{$E(S P)=-940.6375072$} & 6 & 0.656723 & 1.665638 & 0.136604 \\
\hline \multirow{2}{*}{\multicolumn{4}{|c|}{ NImag $=1(-44.6)$}} & 6 & 0.582381 & 3.182344 & -0.121262 \\
\hline & & & & 1 & 1.478209 & 3.655955 & 0.300307 \\
\hline \multirow{2}{*}{\multicolumn{4}{|c|}{ 137. TS-5'E (-940.4598383) }} & 1 & 0.559594 & 3.401772 & -1.195338 \\
\hline & & & & 1 & -0.302580 & 3.633894 & 0.341900 \\
\hline 6 & -2.704959 & -0.747714 & -1.497386 & 6 & 1.881117 & 1.028071 & -0.438741 \\
\hline 16 & -2.233928 & -0.462051 & 0.240812 & 1 & 2.320598 & 1.463951 & -1.334972 \\
\hline 6 & -0.175489 & 0.306458 & -0.006065 & 6 & 2.413900 & -0.145238 & 0.107265 \\
\hline 6 & -0.469383 & 1.721082 & -0.469202 & 6 & 3.679288 & -0.739182 & -0.501673 \\
\hline 6 & -1.804409 & -2.174632 & 0.709296 & 1 & 4.113397 & -0.107208 & -1.283123 \\
\hline 6 & 0.671693 & -0.013358 & 1.214433 & 1 & 4.429506 & -0.895632 & 0.283026 \\
\hline 6 & 2.001783 & 0.122071 & 0.539467 & 1 & 3.458012 & -1.724215 & -0.931815 \\
\hline 6 & 2.430297 & -0.892081 & -0.331898 & 8 & 1.874602 & -0.745054 & 1.090008 \\
\hline 6 & 3.793617 & -0.751592 & -1.004493 & 6 & -1.789939 & 0.923529 & 0.295696 \\
\hline 6 & 0.430923 & 0.846625 & 2.454669 & 8 & -1.807505 & 0.854785 & 1.526027 \\
\hline 8 & 1.731935 & -1.919841 & -0.603376 & 1 & -1.218400 & -3.245490 & 1.143245 \\
\hline 8 & -1.608138 & 2.024248 & -0.810054 & 1 & -1.895924 & -3.073837 & -1.422826 \\
\hline 1 & -2.978598 & 0.219608 & -1.916990 & 6 & -3.077406 & 1.119705 & -0.490890 \\
\hline 1 & -3.557653 & -1.431124 & -1.521774 & 1 & -3.301930 & 2.194450 & -0.529575 \\
\hline 1 & -1.864680 & -1.177119 & -2.051781 & 1 & -3.008735 & 0.761728 & -1.521343 \\
\hline 1 & -2.640843 & -2.829878 & 0.451903 & 1 & -3.905453 & 0.627348 & 0.027741 \\
\hline 1 & -0.892102 & -2.486959 & 0.191788 & 1 & 0.614025 & 1.478772 & 1.217511 \\
\hline 1 & -1.648914 & -2.197702 & 1.789989 & & & & \\
\hline 1 & 0.046912 & -0.402014 & -0.806730 & \multicolumn{4}{|c|}{$E(S P)=-940.6203909$} \\
\hline 1 & -0.602405 & 0.756414 & 2.811659 & \multicolumn{4}{|c|}{ NImag $=1(188.2)$} \\
\hline 1 & 1.096245 & 0.521313 & 3.261621 & & & & \\
\hline 1 & 0.630099 & 1.909086 & 2.276405 & \multicolumn{4}{|c|}{ 139. PC-5' (-940.5313189) } \\
\hline 1 & 2.599149 & 1.013863 & 0.700227 & & & & \\
\hline 1 & 4.409836 & -1.630955 & -0.778766 & 6 & -4.2 & 0.947603 & -0.106626 \\
\hline 1 & 3.668926 & -0.724375 & -2.094719 & 16 & -4.042335 & -0.788337 & 0.438080 \\
\hline 1 & 4.335338 & 0.146773 & -0.691063 & 6 & -3.157284 & -1.472442 & -1.009745 \\
\hline 1 & 0.503023 & -1.066786 & 1.464212 & 8 & -0.826881 & 1.178818 & -1.245335 \\
\hline 6 & 0.640988 & 2.743368 & -0.575000 & 6 & 0.147969 & 1.262287 & -0.505487 \\
\hline 1 & 1.214260 & 2.823686 & 0.352005 & 6 & 0.438890 & 2.543908 & 0.245515 \\
\hline 1 & 1.346104 & 2.442785 & -1.357204 & 6 & 1.071366 & 0.085285 & -0.411899 \\
\hline \multirow[t]{2}{*}{1} & 0.197406 & 3.709582 & -0.829258 & 6 & 2.408347 & 0.100540 & 0.337721 \\
\hline & & & & 6 & 3.566309 & -0.571889 & -0.327183 \\
\hline
\end{tabular}




$\begin{array}{lrrr}6 & 4.929787 & 0.044538 & -0.107129 \\ 6 & 1.260382 & -0.755641 & 0.834747 \\ 6 & 0.581554 & -0.492582 & 2.161953 \\ 8 & 3.410677 & -1.574788 & -1.013707 \\ 1 & -4.878727 & 1.012626 & -0.986135 \\ 1 & -3.252320 & 1.379664 & -0.334500 \\ 1 & -4.691855 & 1.499137 & 0.717199 \\ 1 & -3.787962 & -1.438432 & -1.903552 \\ 1 & -2.233798 & -0.913842 & -1.184224 \\ 1 & -2.918965 & -2.514937 & -0.781619 \\ 1 & 1.074535 & -0.497780 & -1.331968 \\ 1 & -0.460577 & -0.831876 & 2.135310 \\ 1 & 1.094732 & -1.043822 & 2.958156 \\ 1 & 0.584561 & 0.564762 & 2.441682 \\ 1 & 2.639777 & 0.988218 & 0.921397 \\ 1 & 5.157747 & 0.086438 & 0.965687 \\ 1 & 5.696086 & -0.535577 & -0.625517 \\ 1 & 4.938696 & 1.079489 & -0.473422 \\ 1 & 1.377390 & -1.813179 & 0.595814 \\ 1 & 1.367346 & 2.988916 & -0.135307 \\ 1 & -0.384540 & 3.245135 & 0.095108 \\ 1 & 0.584604 & 2.376570 & 1.317742 \\ \text { E (SP) = -940.7018001 } & & \\ \text { NImag = } 0 & & & \end{array}$

